\title{
Synthesis of titanium borylimido compounds supported by diamide-amine ligands and their reactions with alkynes
}

\section{Benjamin A. Clough and Philip Mountford*}

Chemistry Research Laboratory, Department of Chemistry, University of Oxford, Mansfield Road, Oxford OX1 3TA, UK. E-mail: philip.mountford@chem.ox.ac.uk

\section{Supporting Information}

\begin{tabular}{|c|c|}
\hline Contents of the Supporting Information & Page \\
\hline $\begin{array}{l}\text { Figure S1. Displacement ellipsoid plots ( } 20 \% \text { probability) of } \\
\operatorname{Ti}\left(\mathrm{N}_{2}{ }^{\mathrm{R}} \mathrm{N}^{\mathrm{Me}}\right)\left\{\mathrm{NB}\left(\mathrm{NAr}{ }^{\prime} \mathrm{CH}\right)_{2}\right\}\left(\text { py) }\left(\mathrm{R}=\operatorname{Ar}^{\mathrm{F}}(\mathbf{1 0}) \text { or }{ }^{\mathrm{i}} \operatorname{Pr}(\mathbf{1 1})\right)\right.\end{array}$ & S4 \\
\hline $\begin{array}{l}\text { Figure S2. Displacement ellipsoid plot (25\% probability) of } \\
\operatorname{Ti}\left(\mathrm{N}_{2}{ }^{\mathrm{ArF}} \mathrm{N}^{\mathrm{Me}}\right)\left\{\mathrm{N}\left\{\mathrm{B}\left(\mathrm{NAr}{ }^{\prime} \mathrm{CH}\right)_{2}\right\} \mathrm{C}(\mathrm{H}) \mathrm{C}(\mathrm{Tol})\right\}(\mathbf{1 4}) \text {. }\end{array}$ & S5 \\
\hline $\begin{array}{l}\text { Figure S3. Displacement ellipsoid plot (20\% probability) of } \\
\operatorname{Ti}\left\{\mathrm{MeN}\left(\mathrm{CH}_{2} \mathrm{CH}_{2} \mathrm{NAr}^{\mathrm{F}}\right)\left\{\mathrm{CH}_{2} \mathrm{CH}_{2} \mathrm{NC}_{6} \mathrm{~F}_{4} \mathrm{C}\left(\mathrm{Ar}^{\mathrm{F}}\right) \mathrm{CHNB}\left(\mathrm{NAr}{ }^{\prime} \mathrm{CH}\right)_{2}\right\}\right\}(\mathrm{F})(\mathbf{1 5}) \text {. }\end{array}$ & S6 \\
\hline $\begin{array}{l}\text { Figure S4. First order semi-logarithmic plots and Eyring plot for the conversion of } \\
\operatorname{Ti}\left(\mathrm{N}_{2}{ }^{\mathrm{ArF}} \mathrm{N}^{\mathrm{Me}}\right)\left\{\mathrm{N}\left\{\mathrm{B}\left(\mathrm{NAr}{ }^{\prime} \mathrm{CH}\right)_{2}\right\} \mathrm{C}(\mathrm{H}) \mathrm{C}(\mathrm{Tol})\right\}(\mathbf{1 4}) \text { to } \\
\operatorname{Ti}\left\{\mathrm{MeN}\left(\mathrm{CH}_{2} \mathrm{CH}_{2} \mathrm{NAr}^{\mathrm{F}}\right)\left\{\mathrm{CH}_{2} \mathrm{CH}_{2} \mathrm{NC}_{6} \mathrm{~F}_{4} \mathrm{C}(\mathrm{Tol}) \mathrm{CHNB}\left(\mathrm{NAr}{ }^{\prime} \mathrm{CH}\right)_{2}\right\}\right\}(\mathrm{F})(\mathbf{1 7}) \text {. }\end{array}$ & S7 \\
\hline $\begin{array}{l}\text { Figure S5. First order semi-logarithmic plots and Eyring plot for the conversion of } \\
\operatorname{Ti}\left(\mathrm{N}_{2}{ }^{\mathrm{ArF}} \mathrm{N}^{\mathrm{Me}}\right)\left\{\mathrm{N}\left\{\mathrm{B}\left(\mathrm{NAr}^{\prime} \mathrm{CH}\right)_{2}\right\} \mathrm{C}(\mathrm{H}) \mathrm{C}\left(\mathrm{Ar}^{\mathrm{F}}\right)\right\}(\mathbf{1 6}) \text { to } \\
\operatorname{Ti}\left\{\mathrm{MeN}\left(\mathrm{CH}_{2} \mathrm{CH}_{2} \mathrm{NAr}^{\mathrm{F}}\right)\left\{\mathrm{CH}_{2} \mathrm{CH}_{2} \mathrm{NC}_{6} \mathrm{~F}_{4} \mathrm{C}\left(\mathrm{Ar}^{\mathrm{F}}\right) \mathrm{CHNB}\left(\mathrm{NAr} \mathrm{CH}_{2}\right\}\right\}(\mathrm{F})(\mathbf{1 5}) \text {. }\right.\end{array}$ & S8 \\
\hline $\begin{array}{l}\text { Table S1. First order rate constants obtained for the conversion of } \\
\operatorname{Ti}\left(\mathrm{N}_{2}{ }^{\mathrm{ArF}} \mathrm{N}^{\mathrm{Me}}\right)\left\{\mathrm{N}\left\{\mathrm{B}\left(\mathrm{NAr}^{\prime} \mathrm{CH}\right)_{2}\right\} \mathrm{C}(\mathrm{H}) \mathrm{C}(\mathrm{Tol})\right\}(\mathbf{1 4}) \text { to } \\
\mathrm{Ti}\left\{\mathrm{MeN}\left(\mathrm{CH}_{2} \mathrm{CH}_{2} \mathrm{NAr}{ }^{\mathrm{F}}\right)\left\{\mathrm{CH}_{2} \mathrm{CH}_{2} \mathrm{NC}_{6} \mathrm{~F}_{4} \mathrm{C}(\mathrm{Tol}) \mathrm{CHNB}\left(\mathrm{NAr}{ }^{\prime} \mathrm{CH}\right)_{2}\right\}\right\}(\mathrm{F})(\mathbf{1 7}) .\end{array}$ & S9 \\
\hline $\begin{array}{l}\text { Table S2. First order rate constants obtained for the conversion of } \\
\operatorname{Ti}\left(\mathrm{N}_{2}{ }^{\mathrm{ArF}} \mathrm{N}^{\mathrm{Me}}\right)\left\{\mathrm{N}\left\{\mathrm{B}\left(\mathrm{NAr}^{\prime} \mathrm{CH}\right)_{2}\right\} \mathrm{C}(\mathrm{H}) \mathrm{C}\left(\mathrm{Ar}^{\mathrm{F}}\right)\right\}(\mathbf{1 6}) \text { to } \\
\operatorname{Ti}\left\{\mathrm{MeN}\left(\mathrm{CH}_{2} \mathrm{CH}_{2} \mathrm{NAr}^{\mathrm{F}}\right)\left\{\mathrm{CH}_{2} \mathrm{CH}_{2} \mathrm{NC}_{6} \mathrm{~F}_{4} \mathrm{C}\left(\mathrm{Ar}^{\mathrm{F}}\right) \mathrm{CHNB}\left(\mathrm{NAr}{ }^{\prime} \mathrm{CH}\right)_{2}\right\}\right\}(\mathrm{F})(\mathbf{1 5}) \text {. }\end{array}$ & S9 \\
\hline
\end{tabular}


Figure S6. Displacement ellipsoid plot (25\% probability) of cis $-\mathrm{Ti}\left(\mathrm{N}_{2}{ }^{\mathrm{iPr}} \mathrm{N}^{\mathrm{Me}}\right)\left\{\mathrm{N}\left\{\mathrm{B}\left(\mathrm{NAr}{ }^{\prime} \mathrm{CH}\right)_{2}\right\} \mathrm{C}(\mathrm{H}) \mathrm{C}(\mathrm{Tol})\right\}$ (cis-18).

Figure S7. Plot of the sum of $\left[\mathrm{Ti}\left(\mathrm{N}_{2} \mathrm{~N}^{\mathrm{py}}\right)\left\{\mathrm{NHB}\left(\mathrm{NAr}{ }^{\prime} \mathrm{CH}\right)_{2}\right\}\left(\mathrm{CCAr}^{\mathrm{F}}\right)\right]([\mathbf{2 3}])$ and $\left[\mathrm{Ti}\left(\mathrm{N}_{2} \mathrm{~N}^{\mathrm{py}}\right)\left\{\mathrm{N}\left\{\mathrm{B}\left(\mathrm{NAr}{ }^{\prime} \mathrm{CH}\right)_{2}\right\} \mathrm{C}(\mathrm{H}) \mathrm{C}(\mathrm{Tol})\right\}\right]([\mathbf{2 4}])$ vs time, and similarly for [23-d $\left.\boldsymbol{d}_{\mathbf{1}}\right]$ and [24-di].

Figure S8. First order semi-logarithmic plots for the conversion of S12 $\mathrm{Ti}\left(\mathrm{N}_{2} \mathrm{~N}^{\mathrm{py}}\right)\left\{\mathrm{NHB}\left(\mathrm{NAr}^{\prime} \mathrm{CH}\right)_{2}\right\}\left(\mathrm{CCAr}^{\mathrm{F}}\right)(\mathbf{2 3})$ to $\mathrm{Ti}\left(\mathrm{N}_{2} \mathrm{~N}^{\mathrm{py}}\right)\left\{\mathrm{N}\left\{\mathrm{B}\left(\mathrm{NAr}^{\prime} \mathrm{CH}\right)_{2}\right\} \mathrm{C}(\mathrm{H}) \mathrm{C}\left(\mathrm{Ar}^{\mathrm{F}}\right)\right\}$ (24), and 23-d $d_{1}$ to 24-d1.

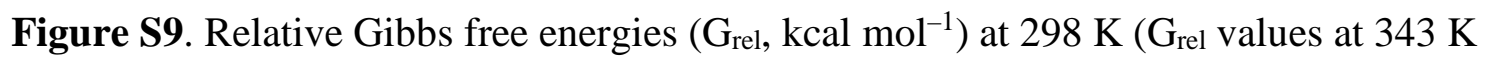
S13 in parentheses) for the DFT computed, gas phase reactions of $\mathrm{Ti}\left(\mathrm{N}_{2}{ }^{\mathrm{SiH} 3} \mathrm{~N}^{\mathrm{py}}\right)\left\{\mathrm{NB}(\mathrm{NMeCH})_{2}\right\}$ (py) (1Q) with $\mathrm{Ar}^{\mathrm{F}} \mathrm{CCH}$ and $\mathrm{Ar}^{\mathrm{F}} \mathrm{CCD}$

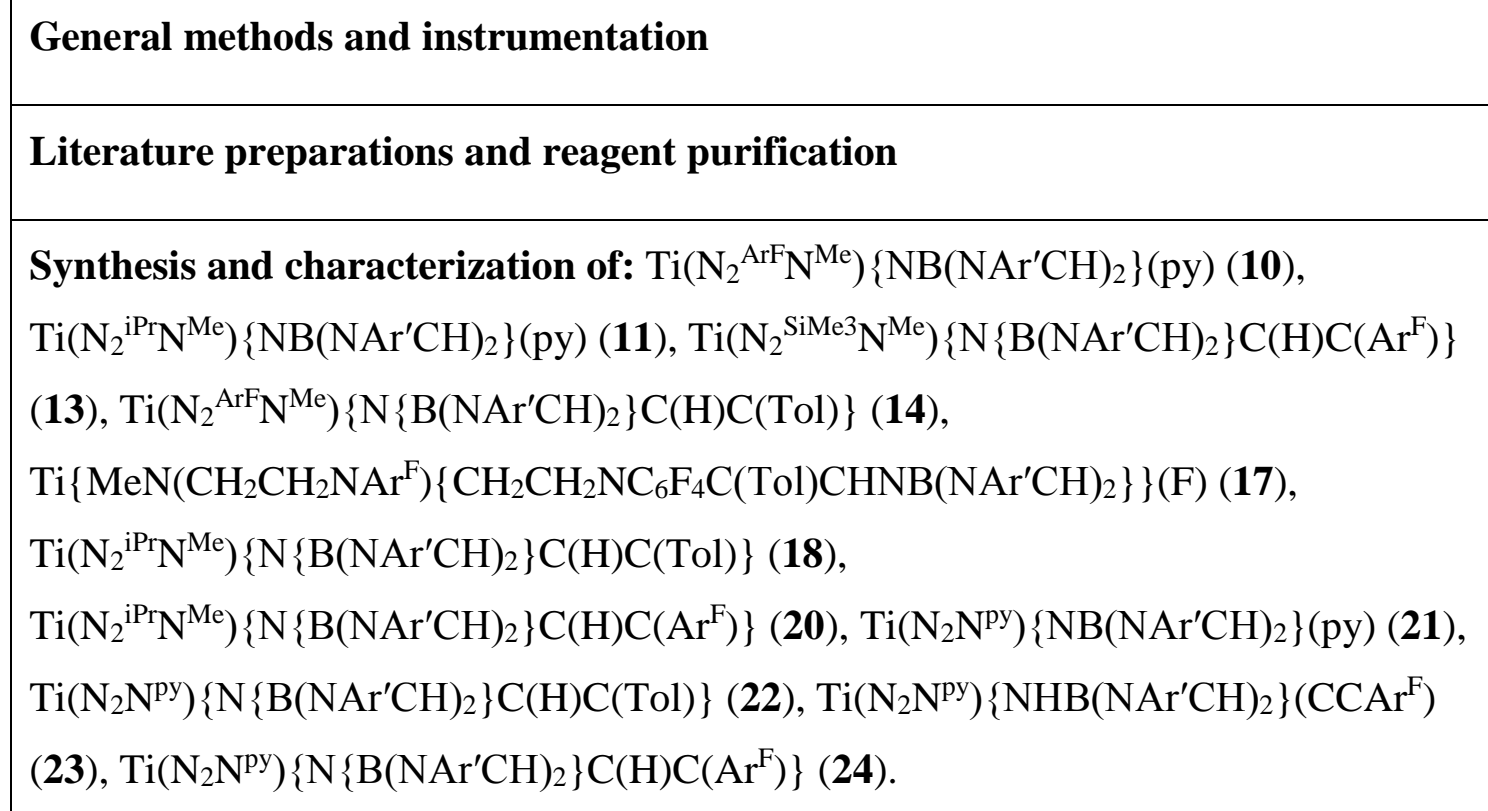

NMR tube scale experiments: synthesis of $\operatorname{Ti}\left(\mathrm{N}_{2}{ }^{\mathrm{ArF}} \mathrm{N}^{\mathrm{Me}}\right)\left\{\mathrm{N}\left\{\mathrm{B}\left(\mathrm{NAr}{ }^{\prime} \mathrm{CH}\right)_{2}\right\} \mathrm{C}(\mathrm{H}) \mathrm{C}\left(\mathrm{Ar}^{\mathrm{F}}\right)\right\}$ (16), synthesis of $\operatorname{Ti}\left\{\mathrm{MeN}\left(\mathrm{CH}_{2} \mathrm{CH}_{2} \mathrm{NAr}^{\mathrm{F}}\right)\left\{\mathrm{CH}_{2} \mathrm{CH}_{2} \mathrm{NC}_{6} \mathrm{~F}_{4} \mathrm{C}(\mathrm{Tol}) \mathrm{CHNB}\left(\mathrm{NAr}{ }^{\prime} \mathrm{CH}\right)_{2}\right\}\right\}(\mathrm{F})(\mathbf{1 7})$, kinetic measurements for the conversion of $\mathrm{Ti}_{(}\left(\mathrm{N}_{2}{ }^{\mathrm{ArF}} \mathrm{N}^{\mathrm{Me}}\right)\left\{\mathrm{N}\left\{\mathrm{B}\left(\mathrm{NAr}{ }^{\prime} \mathrm{CH}\right)_{2}\right\} \mathrm{C}(\mathrm{H}) \mathrm{C}(\mathrm{Tol})\right\}$ (14) to $\mathrm{Ti}\left\{\mathrm{MeN}\left(\mathrm{CH}_{2} \mathrm{CH}_{2} \mathrm{NAr}^{\mathrm{F}}\right)\left\{\mathrm{CH}_{2} \mathrm{CH}_{2} \mathrm{NC}_{6} \mathrm{~F}_{4} \mathrm{C}(\mathrm{Tol}) \mathrm{CHNB}\left(\mathrm{NAr}{ }^{\prime} \mathrm{CH}\right)_{2}\right\}\right\}(\mathrm{F})(\mathbf{1 7})$, kinetic measurements for the conversion of $\mathrm{Ti}\left(\mathrm{N}_{2}{ }^{\mathrm{ArF}} \mathrm{N}^{\mathrm{Me}}\right)\left\{\mathrm{N}\left\{\mathrm{B}\left(\mathrm{NAr}{ }^{\prime} \mathrm{CH}\right)_{2}\right\} \mathrm{C}(\mathrm{H}) \mathrm{C}\left(\mathrm{Ar}^{\mathrm{F}}\right)\right\}$ (16) to $\mathrm{Ti}\left\{\mathrm{MeN}\left(\mathrm{CH}_{2} \mathrm{CH}_{2} \mathrm{NAr}^{\mathrm{F}}\right)\left\{\mathrm{CH}_{2} \mathrm{CH}_{2} \mathrm{NC}_{6} \mathrm{~F}_{4} \mathrm{C}\left(\mathrm{Ar}^{\mathrm{F}}\right) \mathrm{CHNB}\left(\mathrm{NAr}{ }^{\prime} \mathrm{CH}\right)_{2}\right\}\right\}(\mathrm{F})$ (15), synthesis of $\mathrm{Ti}\left(\mathrm{N}_{2}{ }^{\mathrm{iPr}} \mathrm{N}^{\mathrm{Me}}\right)\left\{\mathrm{N}\left\{\mathrm{B}\left(\mathrm{NAr}{ }^{\prime} \mathrm{CH}\right)_{2}\right\} \mathrm{C}(\mathrm{H}) \mathrm{C}\left(\mathrm{Ar}^{\mathrm{F}}\right)\right\}(\mathbf{2 0})$, kinetic isotope effect measurements 


\begin{tabular}{|c|c|}
\hline 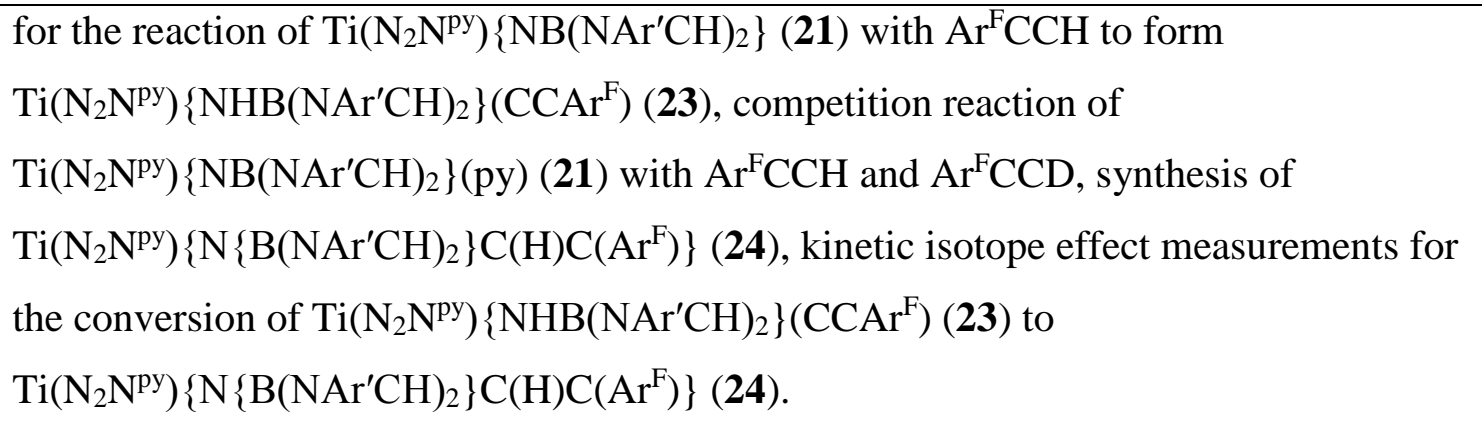 & \\
\hline$X$-ray data collection and processing procedures & S26 \\
\hline Computational Details & S26 \\
\hline $\begin{array}{l}\text { Table S3. Total SCF Energies, thermal corrections to enthalpies, thermal corrections to } \\
\text { Gibbs free energies for gas phase DFT reactions. }\end{array}$ & $\mathrm{S} 28$ \\
\hline $\begin{array}{l}\text { Table S4. Total SCF energies and sums of SCF energy and thermal corrections to } \\
\text { enthalpies or Gibbs free energies for DFT calculations on the full experimental } \\
\text { compounds. }\end{array}$ & S30 \\
\hline References for the SI & S32 \\
\hline
\end{tabular}



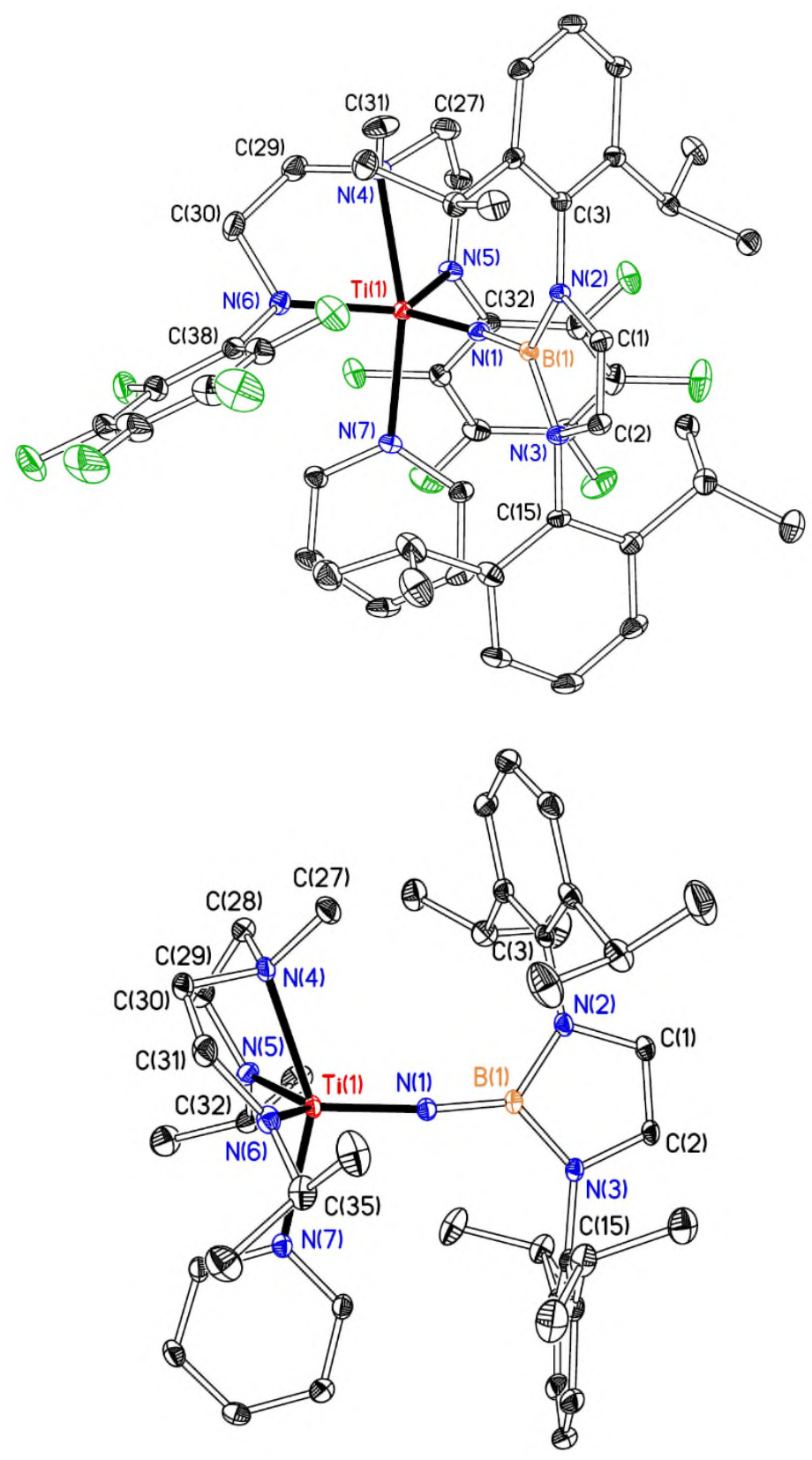

Figure S1. Displacement ellipsoid plots (20\% probability) of $\operatorname{Ti}\left(\mathrm{N}_{2}{ }^{\mathrm{R}} \mathrm{N}^{\mathrm{Me}}\right)\left\{\mathrm{NB}\left(\mathrm{NAr}{ }^{\prime} \mathrm{CH}\right)_{2}\right\}(\mathrm{py})(\mathrm{R}=$ $\operatorname{Ar}^{\mathrm{F}}\left(\mathbf{1 0}\right.$, top) and ${ }^{\mathrm{i}} \operatorname{Pr}(\mathbf{1 1}$, bottom). 


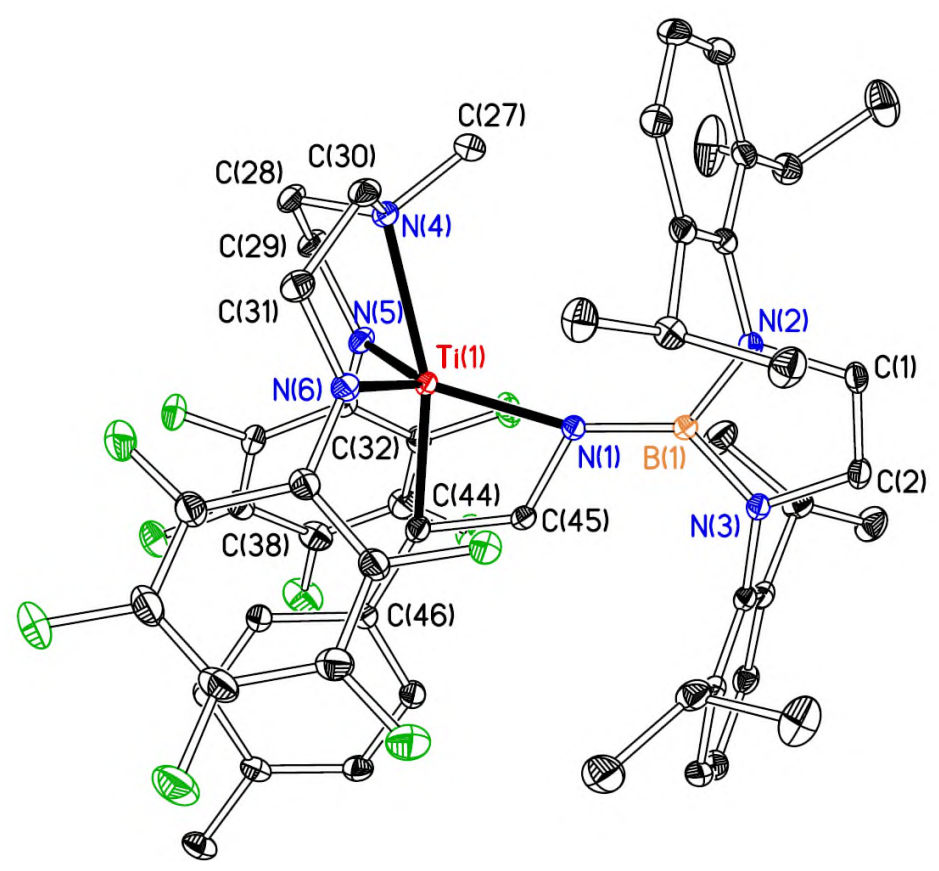

Figure S2. Displacement ellipsoid plot (25\% probability) of $\mathrm{Ti}\left(\mathrm{N}_{2}{ }^{\mathrm{ArF}} \mathrm{N}^{\mathrm{Me}}\right)\left\{\mathrm{N}\left\{\mathrm{B}\left(\mathrm{NAr}{ }^{\prime} \mathrm{CH}\right)_{2}\right\} \mathrm{C}(\mathrm{H}) \mathrm{C}(\mathrm{Tol})\right\}$ (14). H atoms are omitted for clarity. Selected bond distances $(\AA)$ and angles $\left({ }^{\circ}\right)$ : $\operatorname{Ti}(1)-\mathrm{N}(1)$ 1.9833(13), $\operatorname{Ti}(1)-\mathrm{N}(4)$ 2.2530(14), $\mathrm{Ti}(1)-\mathrm{N}(5)$ 1.9944(15), $\mathrm{Ti}(1)-\mathrm{N}(6)$ 1.9882(15), Ti(1)-C(44) 1.9522(17), N(1)-B(1) 1.443(2), N(1)-C(45) 1.402(2), C(44)$\mathrm{C}(45) \quad 1.389(2), \quad \mathrm{N}(1)-\mathrm{Ti}(1)-\mathrm{C}(44) \quad 77.19(6), \quad \mathrm{Ti}(1)-\mathrm{N}(1)-\mathrm{B}(1) \quad 161.16(11), \quad \mathrm{Ti}(1)-\mathrm{C}(44)-\mathrm{C}(45)$ 80.31(10), N(1)-C(45)-C(44) 123.18(15) ( $\tau=0.69)$. 


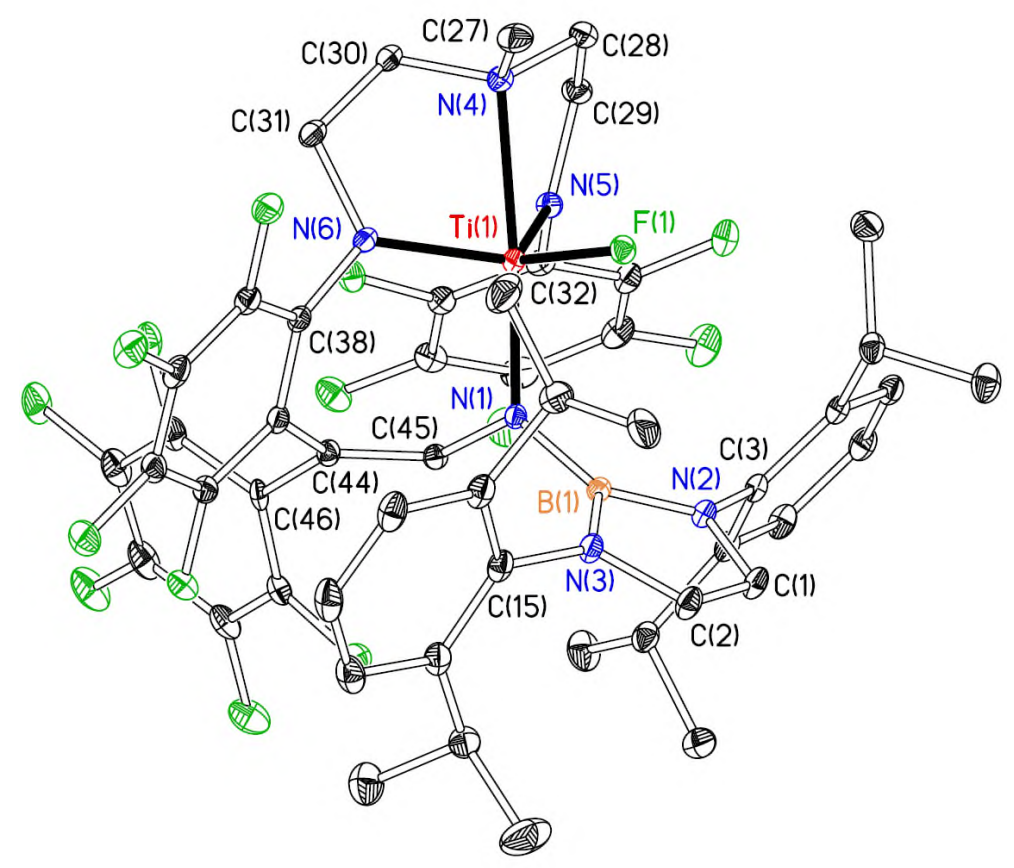

Figure S3. Displacement ellipsoid plot (20\% probability) of $\mathrm{Ti}\left\{\mathrm{MeN}\left(\mathrm{CH}_{2} \mathrm{CH}_{2} \mathrm{NAr}^{\mathrm{F}}\right)\left\{\mathrm{CH}_{2} \mathrm{CH}_{2} \mathrm{NC}_{6} \mathrm{~F}_{4} \mathrm{C}\left(\mathrm{Ar}^{\mathrm{F}}\right) \mathrm{CHNB}\left(\mathrm{NAr}{ }^{\prime} \mathrm{CH}\right)_{2}\right\}\right\}(\mathrm{F})(\mathbf{1 5})$. H atoms are omitted for clarity. Selected bond lengths $(\AA)$ and angles $\left(^{\circ}\right)$ : $\mathrm{Ti}(1)-\mathrm{N}(1)$ 1.9300(17), $\mathrm{Ti}(1)-\mathrm{N}(4)$ 2.2667(19), Ti(1)-N(5) 1.9759(18), Ti(1)-N(6) 1.9466(18), Ti(1)-F(1) 1.8016(12), N(1)-B(1) 1.479(3), N(1)$\mathrm{C}(45)$ 1.405(2), C(44)-C(45) 1.343(3), Ti(1)-N(1)-B(1) 127.01(13) $(\tau=0.93)$. 

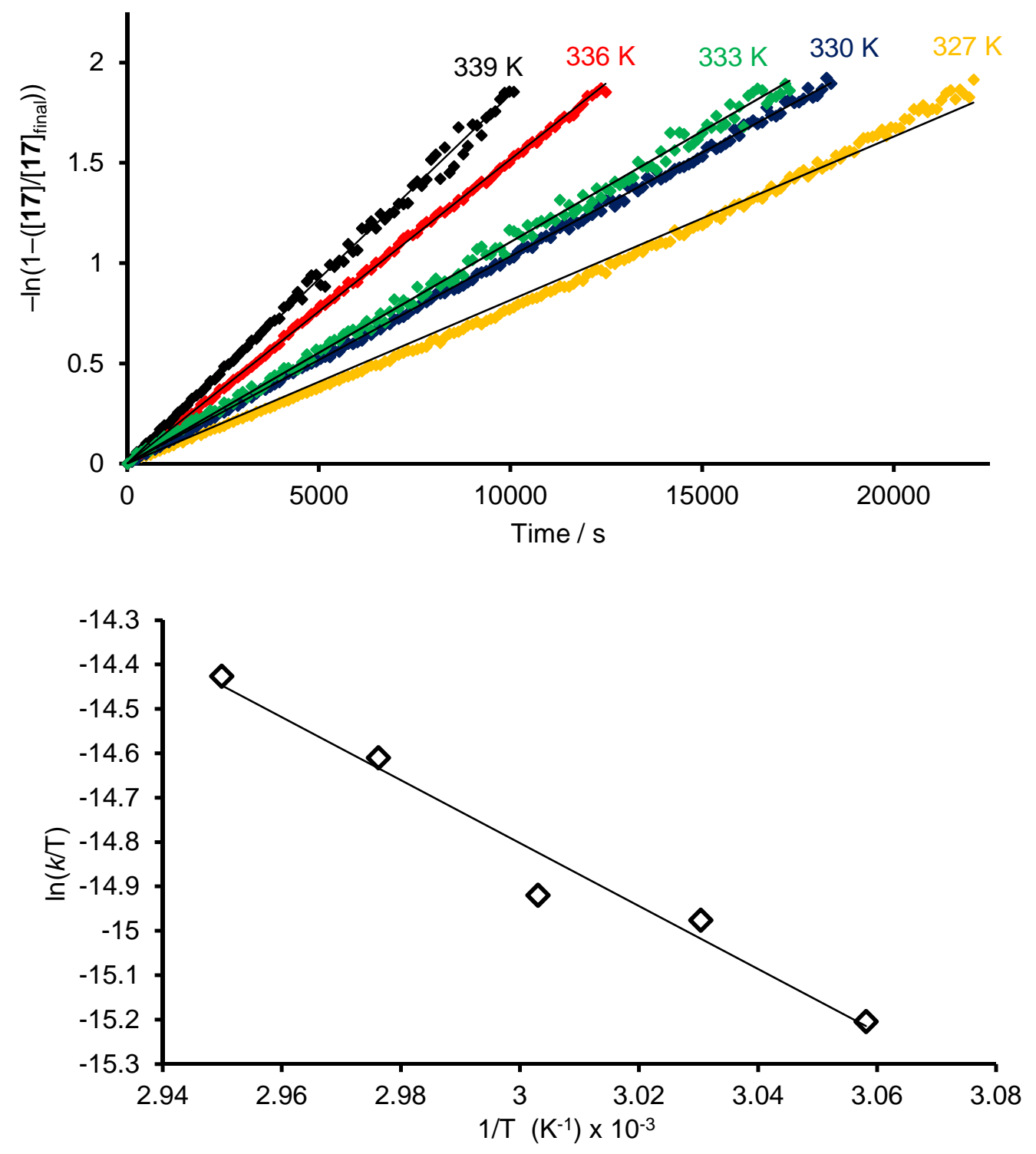

Figure S4. Top: first order semi-logarithmic plots for the conversion of $\mathrm{Ti}\left(\mathrm{N}_{2}{ }^{\mathrm{ArF}} \mathrm{N}^{\mathrm{Me}}\right)\left\{\mathrm{N}\left\{\mathrm{B}\left(\mathrm{NAr}{ }^{\prime} \mathrm{CH}\right)_{2}\right\} \mathrm{C}(\mathrm{H}) \mathrm{C}(\mathrm{Tol})\right\}$

$\mathrm{Ti}\left\{\mathrm{MeN}\left(\mathrm{CH}_{2} \mathrm{CH}_{2} \mathrm{NAr}^{\mathrm{F}}\right)\left\{\mathrm{CH}_{2} \mathrm{CH}_{2} \mathrm{NC}_{6} \mathrm{~F}_{4} \mathrm{C}(\mathrm{Tol}) \mathrm{CHNB}\left(\mathrm{NAr}{ }^{\prime} \mathrm{CH}\right)_{2}\right\}\right\}(\mathrm{F})(\mathbf{1 7})$ in $\mathrm{C}_{6} \mathrm{D}_{6}$. (avg. $r^{2}=0.998$, range 0.995 - 0.999). Bottom: Eyring plot for the conversion of $\mathrm{Ti}\left(\mathrm{N}_{2}{ }^{\mathrm{ArF}} \mathrm{N}^{\mathrm{Me}}\right)\left\{\mathrm{N}\left\{\mathrm{B}\left(\mathrm{NAr}{ }^{\prime} \mathrm{CH}\right)_{2}\right\} \mathrm{C}(\mathrm{H}) \mathrm{C}(\mathrm{Tol})\right\}$

to $\mathrm{Ti}\left\{\mathrm{MeN}\left(\mathrm{CH}_{2} \mathrm{CH}_{2} \mathrm{NAr}^{\mathrm{F}}\right)\left\{\mathrm{CH}_{2} \mathrm{CH}_{2} \mathrm{NC}_{6} \mathrm{~F}_{4} \mathrm{C}(\mathrm{Tol}) \mathrm{CHNB}\left(\mathrm{NAr}{ }^{\prime} \mathrm{CH}\right)_{2}\right\}\right\}(\mathrm{F})$ (17) in $\mathrm{C}_{6} \mathrm{D}_{6}$. Activation parameters derived from linear regression analysis $\left(r^{2}=0.969\right): \Delta \mathrm{H}^{\ddagger}=14.1(1.5) \mathrm{kcal} \mathrm{mol}^{-1} ; \Delta \mathrm{S}^{\ddagger}=-$ 34.3(4.4) $\mathrm{cal} \mathrm{mol}^{-1} \mathrm{~K}^{-1} ; \Delta \mathrm{G}_{333^{\ddagger}}=25.5(1.5) \mathrm{kcal} \mathrm{mol}^{-1}$. The experiment at $333 \mathrm{~K}$ was repeated with double $[\mathbf{1 4}]_{0}$, giving $\ln (k / \mathrm{T})=-14.8\left(1 / \mathrm{T}=3.00 \times 10^{-3} \mathrm{~K}^{-1}\right)$. 

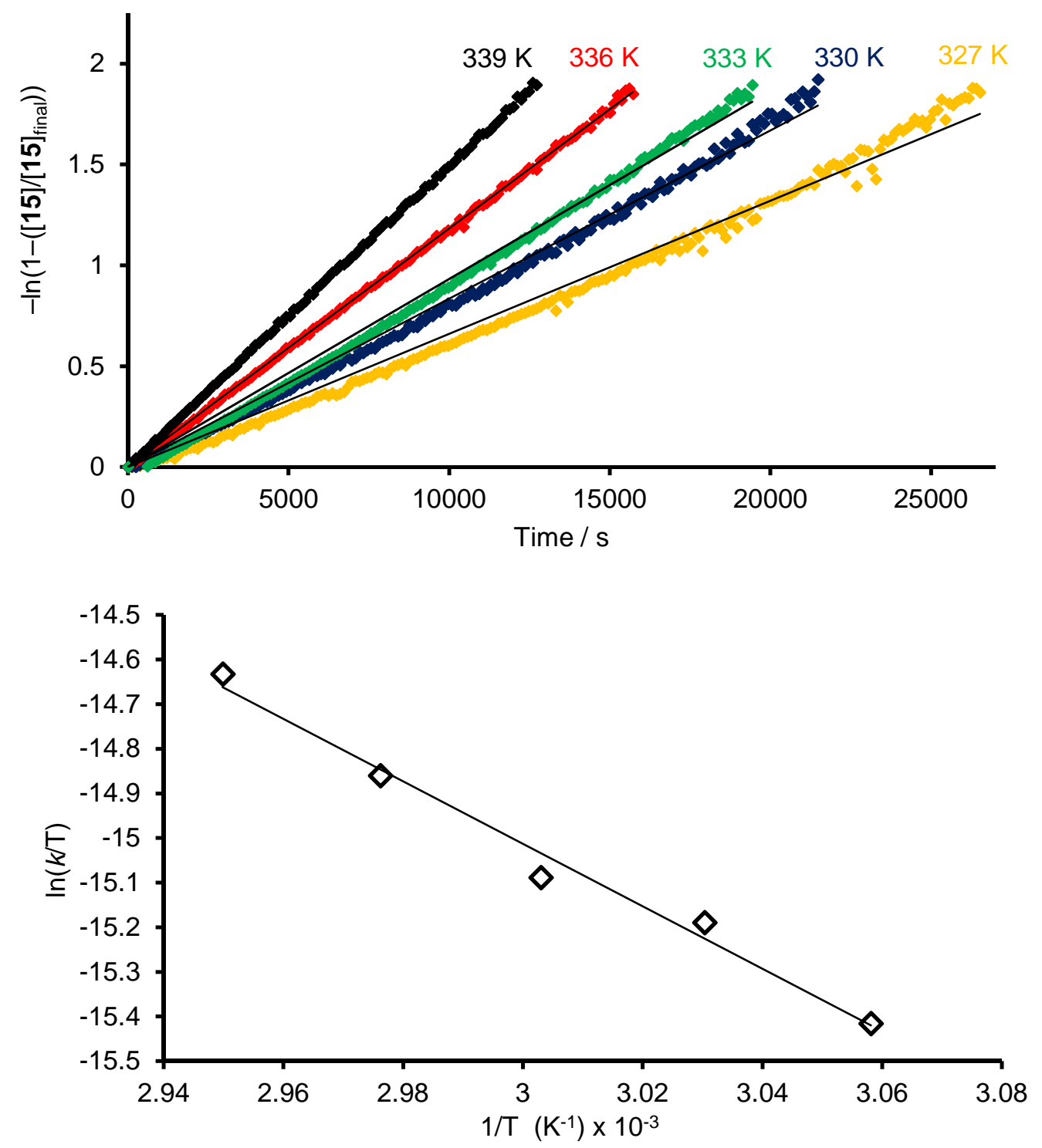

Figure S5. Top: first order semi-logarithmic plots for the conversion of $\mathrm{Ti}\left(\mathrm{N}_{2}{ }^{\mathrm{ArF}} \mathrm{N}^{\mathrm{Me}}\right)\left\{\mathrm{N}\left\{\mathrm{B}\left(\mathrm{NAr}{ }^{\prime} \mathrm{CH}\right)_{2}\right\} \mathrm{C}(\mathrm{H}) \mathrm{C}\left(\mathrm{Ar}^{\mathrm{F}}\right)\right\}$

$\mathrm{Ti}\left\{\mathrm{MeN}\left(\mathrm{CH}_{2} \mathrm{CH}_{2} \mathrm{NAr}^{\mathrm{F}}\right)\left\{\mathrm{CH}_{2} \mathrm{CH}_{2} \mathrm{NC}_{6} \mathrm{~F}_{4} \mathrm{C}\left(\mathrm{Ar}^{\mathrm{F}}\right) \mathrm{CHNB}\left(\mathrm{NAr}{ }^{\prime} \mathrm{CH}\right)_{2}\right\}\right\}(\mathrm{F})$ (15) in $\mathrm{C}_{6} \mathrm{D}_{6}$. (avg. $r^{2}=$ 0.996, range 0.990 - 0.999). Bottom: Eyring plot for the conversion of $\mathrm{Ti}\left(\mathrm{N}_{2}{ }^{\mathrm{ArF}} \mathrm{N}^{\mathrm{Me}}\right)\left\{\mathrm{N}\left\{\mathrm{B}\left(\mathrm{NAr}{ }^{\prime} \mathrm{CH}\right)_{2}\right\} \mathrm{C}(\mathrm{H}) \mathrm{C}\left(\mathrm{Ar}^{\mathrm{F}}\right)\right\}$

to

$\mathrm{Ti}\left\{\mathrm{MeN}\left(\mathrm{CH}_{2} \mathrm{CH}_{2} \mathrm{NAr}^{\mathrm{F}}\right)\left\{\mathrm{CH}_{2} \mathrm{CH}_{2} \mathrm{NC}_{6} \mathrm{~F}_{4} \mathrm{C}\left(\mathrm{Ar}^{\mathrm{F}}\right) \mathrm{CHNB}\left(\mathrm{NAr}{ }^{\prime} \mathrm{CH}\right)_{2}\right\}\right\}(\mathrm{F})$ (15) in $\mathrm{C}_{6} \mathrm{D}_{6}$. Activation parameters derived from linear regression analysis $\left(r^{2}=0.985\right): \Delta \mathrm{H}^{\ddagger}=13.9(1.0) \mathrm{kcal} \mathrm{mol}^{-1} ; \Delta \mathrm{S}^{\ddagger}=-$ 35.3(2.9) $\mathrm{cal} \mathrm{mol}^{-1} \mathrm{~K}^{-1} ; \Delta \mathrm{G}_{333^{\ddagger}}=25.7(1.0) \mathrm{kcal} \mathrm{mol}^{-1}$. The experiment at $336 \mathrm{~K}$ was repeated with double $[\mathbf{1 6}]_{0}$, giving $\ln (k / \mathrm{T})=-14.8\left(1 / \mathrm{T}=2.98 \times 10^{-3} \mathrm{~K}^{-1}\right)$. 
Table S1. First order rate constants obtained for the conversion of $\mathrm{Ti}\left(\mathrm{N}_{2}{ }^{\mathrm{ArF}} \mathrm{N}^{\mathrm{Me}}\right)\left\{\mathrm{N}\left\{\mathrm{B}\left(\mathrm{NAr}{ }^{\prime} \mathrm{CH}\right)_{2}\right\} \mathrm{C}(\mathrm{H}) \mathrm{C}(\mathrm{Tol})\right\}$ (14) to $\mathrm{Ti}\left\{\mathrm{MeN}\left(\mathrm{CH}_{2} \mathrm{CH}_{2} \mathrm{NAr}^{\mathrm{F}}\right)\left\{\mathrm{CH}_{2} \mathrm{CH}_{2} \mathrm{NC}_{6} \mathrm{~F}_{4} \mathrm{C}(\mathrm{Tol})\right.\right.$ $\left.\mathrm{CHNB}\left(\mathrm{NAr} \mathrm{CH}_{2}\right\}\right\}(\mathrm{F})(\mathbf{1 7})$. Unless otherwise stated, $[\mathbf{1 4}]_{0}=0.026 \mathrm{~mol} \mathrm{dm}^{-3}$.

\begin{tabular}{|c|c|c|}
\hline Temperature / K & Notes & $k_{\mathrm{obs}} / 10^{-5} \mathrm{~s}^{-1}$ \\
\hline 327 & & $8.15(2)$ \\
\hline 330 & & $10.34(1)$ \\
\hline 333 & & $11.04(3)$ \\
\hline 333 & {$[\mathbf{1 4}]_{0}=0.052 \mathrm{~mol} \mathrm{dm}^{-3}$} & $12.72(2)$ \\
\hline 336 & & $15.18(2)$ \\
\hline 339 & & $18.41(6)$ \\
\hline
\end{tabular}

Table S2. First order rate constants obtained for the conversion of $\operatorname{Ti}\left(\mathrm{N}_{2}{ }^{\mathrm{ArF}} \mathrm{N}^{\mathrm{Me}}\right)\left\{\mathrm{N}\left\{\mathrm{B}\left(\mathrm{NAr}{ }^{\prime} \mathrm{CH}\right)_{2}\right\} \mathrm{C}(\mathrm{H}) \mathrm{C}\left(\mathrm{Ar}^{\mathrm{F}}\right)\right\}$ (16) to $\mathrm{Ti}\left\{\mathrm{MeN}\left(\mathrm{CH}_{2} \mathrm{CH}_{2} \mathrm{NAr}^{\mathrm{F}}\right)\left\{\mathrm{CH}_{2} \mathrm{CH}_{2} \mathrm{NC}_{6} \mathrm{~F}_{4} \mathrm{C}\left(\mathrm{Ar}^{\mathrm{F}}\right)\right.\right.$ $\left.\left.\mathrm{CHNB}\left(\mathrm{NAr}{ }^{\prime} \mathrm{CH}\right)_{2}\right\}\right\}(\mathrm{F})(\mathbf{1 5})$. Unless otherwise stated, $[\mathbf{1 6}]_{0}=0.026 \mathrm{~mol} \mathrm{dm}^{-3}$

\begin{tabular}{|c|c|c|}
\hline Temperature / K & Notes & $k_{\text {obs }} / 10^{-5} \mathrm{~s}^{-1}$ \\
\hline 327 & & $6.60(2)$ \\
\hline 330 & & $8.35(2)$ \\
\hline 333 & & $9.32(3)$ \\
\hline 336 & & $11.82(1)$ \\
\hline 336 & {$[\mathbf{1 6}]_{0}=0.052 \mathrm{~mol} \mathrm{dm}^{-3}$} & $12.63(2)$ \\
\hline 339 & & $14.97(1)$ \\
\hline
\end{tabular}




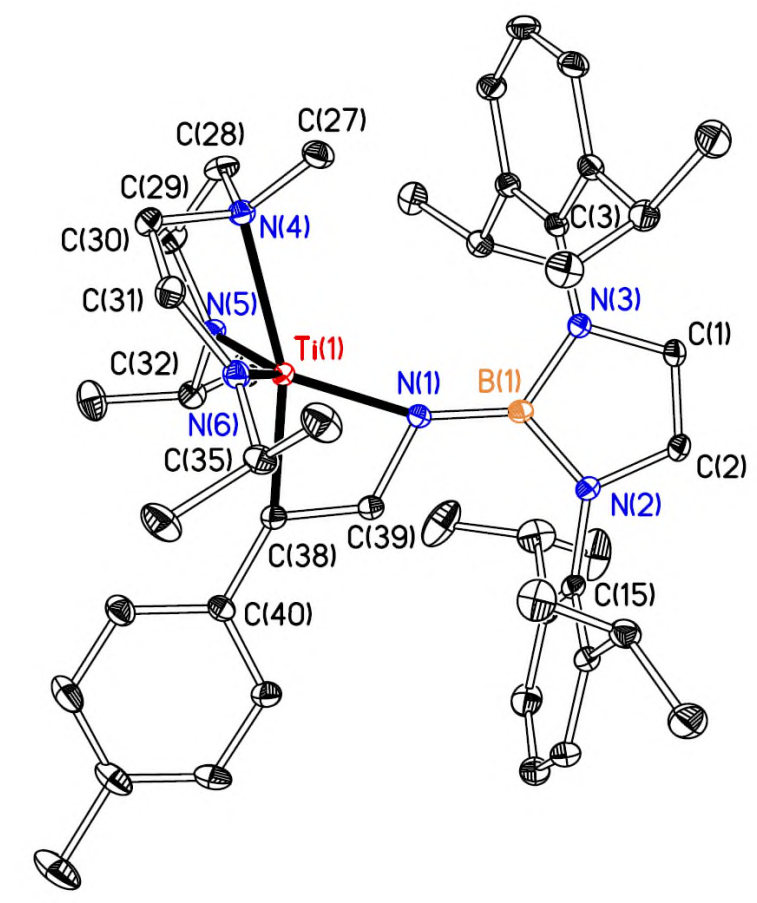

Figure S6. Displacement ellipsoid plot (25\% probability) of cis- $\mathrm{Ti}\left(\mathrm{N}_{2}{ }^{\left.{ }^{\mathrm{Pr}} \mathrm{N}^{\mathrm{Me}}\right)}\left\{\mathrm{N}\left\{\mathrm{B}\left(\mathrm{NAr}{ }^{\prime} \mathrm{CH}\right)_{2}\right\} \mathrm{C}(\mathrm{H}) \mathrm{C}(\mathrm{Tol})\right\}\right.$ (cis-18). H atoms are omitted for clarity. Selected bond distances $(\AA)$ and angles $\left({ }^{\circ}\right) \mathrm{Ti}(1)-\mathrm{N}(1)$ 1.999(2) [1.996(2)], Ti(1)-N(4) 2.294(2) [2.301(2)], $\mathrm{Ti}(1)-\mathrm{N}(5)$ 1.963(3) [1.943(3)], $\mathrm{Ti}(1)-\mathrm{N}(6)$ 1.957(2) [1.953(3)], Ti(1)-C(38) 1.998(3) [2.007(3)], $\mathrm{N}(1)-\mathrm{B}(1)$ 1.432(4) [1.426(4)], N(1)-C(39) 1.433(3) [1.424(4)], C(38)-C(39) 1.358(4) [1.357(4)], $\mathrm{N}(1)-\mathrm{Ti}(1)-\mathrm{C}(38)$ 75.34(10) [75.13(10)], Ti(1)-N(1)-B(1) 160.17(18) [159.24(19)], Ti(1)-C(38)$\mathrm{C}(39), 82.13(17)$ [81.65(18)], N(1)-C(39)-C(38) 122.1(2) [122.6(3)]. Values in brackets are for the second crystallographically independent molecule $(\tau=0.58[0.63])$. 

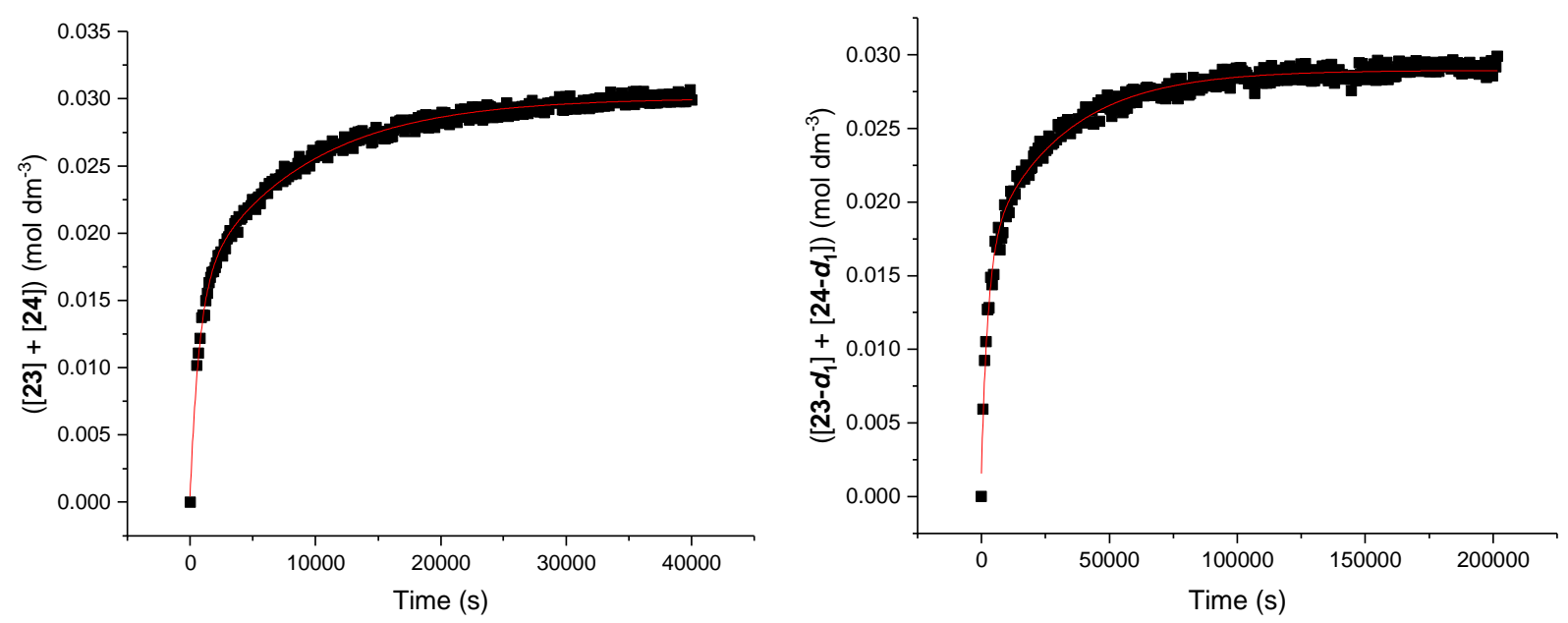

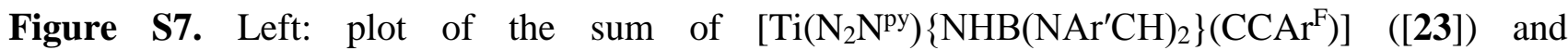
$\left[\mathrm{Ti}\left(\mathrm{N}_{2} \mathrm{~N}^{\mathrm{py}}\right)\left\{\mathrm{N}\left\{\mathrm{B}\left(\mathrm{NAr}{ }^{\prime} \mathrm{CH}\right)_{2}\right\} \mathrm{C}(\mathrm{H}) \mathrm{C}(\mathrm{Tol})\right\}\right]([\mathbf{2 4}])$ vs time. $[\mathbf{2 1}]_{0}=\left[\mathrm{Ar}^{\mathrm{F}} \mathrm{CCH}\right]_{0}=0.0299 \mathrm{~mol} \mathrm{dm}^{-3}$ and $\mathrm{T}=298 \mathrm{~K}$. The best-fit line $\left(r^{2}=0.9928\right)$ shown gave an initial rate of $19.2(1) \times 10^{-6} \mathrm{~mol} \mathrm{dm}^{-3} \mathrm{~s}^{-1}$.

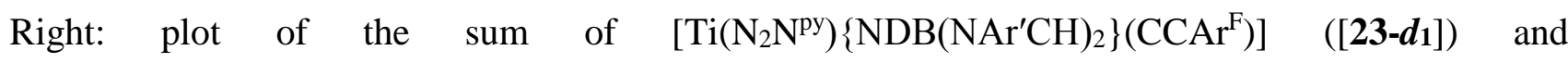
$\left[\mathrm{Ti}\left(\mathrm{N}_{2} \mathrm{~N}^{\mathrm{py}}\right)\left\{\mathrm{N}\left\{\mathrm{B}\left(\mathrm{NAr}{ }^{\prime} \mathrm{CH}\right)_{2}\right\} \mathrm{C}(\mathrm{D}) \mathrm{C}(\mathrm{Tol})\right\}\right]\left(\left[\mathbf{2 4 - d _ { 1 } ] )} v s\right.\right.$ time. The best-fit line $\left(r^{2}=0.9847\right)$ shown gave an initial rate of $3.9(1) \times 10^{-6} \mathrm{~mol} \mathrm{dm}^{-3} \mathrm{~s}^{-1}$.

Note: In order to avoid the effects of the build-up of the released pyridine as the reaction progressed, the initial rates method was used to effectively measure the rate of the reaction, $\mathrm{d}[23] / \mathrm{d} t$ at time $=0$, $t_{0}$. The reaction of $\mathrm{Ar}^{\mathrm{F}} \mathrm{CCH}$ with 21 was monitored by a ${ }^{1} \mathrm{H}$ NMR array at $298 \mathrm{~K}$. A small $(<10$ molar\%) amount of the thermodynamic metallacycle product $\mathbf{2 4}$ was also observed to form, though remaining as a constant proportion of the product mixture as the reaction proceeded. It was shown independently that $\mathbf{2 3}$ does not itself convert to $\mathbf{2 4}$ under these conditions and so $\mathrm{d}[\mathbf{2 3}] / \mathrm{d} t$ is independent of $\mathrm{d}[\mathbf{2 4}] / \mathrm{d} t$. Therefore, a composite initial rate was measured, and was then scaled to the correct proportion of the acetylide complex $\mathbf{2 3}$ in the mixture, to give an initial rate reflecting only the formation of $\mathbf{2 3}$. The graph of $([\mathbf{2 3}]+[\mathbf{2 4}]) v s$ time gave the non-linear plot drawn above in Fig. S7 Graphical software (Origin) was used to fit a curve to all of the data points, thus describing the evolution of product throughout the kinetic study. 


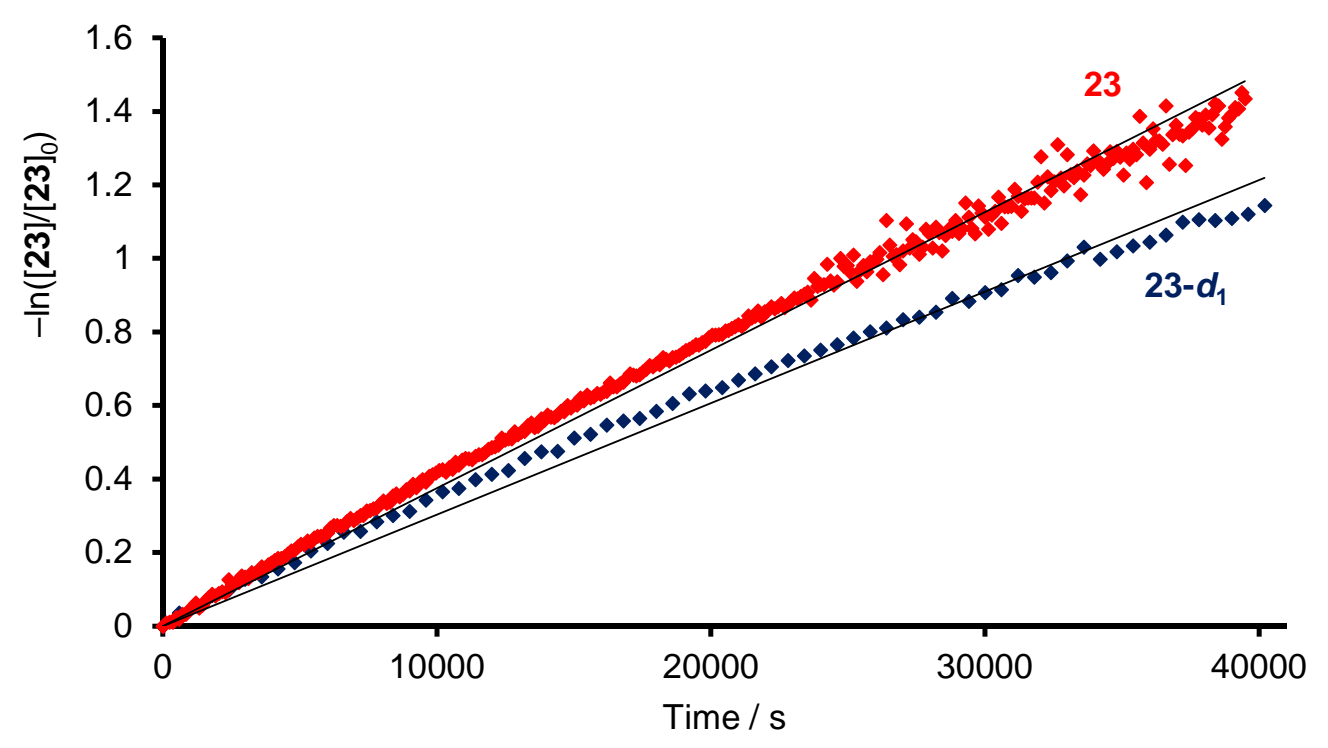

Figure S8. First order semi-logarithmic plots for the conversion of $\mathrm{Ti}\left(\mathrm{N}_{2} \mathrm{~N}^{\mathrm{py}}\right)\left\{\mathrm{NHB}\left(\mathrm{NAr}^{\prime} \mathrm{CH}\right)_{2}\right\}\left(\mathrm{CCAr}^{\mathrm{F}}\right)(\mathbf{2 3})$ to $\mathrm{Ti}\left(\mathrm{N}_{2} \mathrm{~N}^{\mathrm{py}}\right)\left\{\mathrm{N}\left\{\mathrm{B}\left(\mathrm{NAr}^{\prime} \mathrm{CH}\right)_{2}\right\} \mathrm{C}(\mathrm{H}) \mathrm{C}\left(\mathrm{Ar}^{\mathrm{F}}\right)\right\}$ (24), and 23$\boldsymbol{d}_{\mathbf{1}}$ to $24-\boldsymbol{d}_{1}$, at $343 \mathrm{~K}$ in $\mathrm{C}_{6} \mathrm{D}_{6}\left(r^{2}=0.991\right.$ and 0.982$)$. 


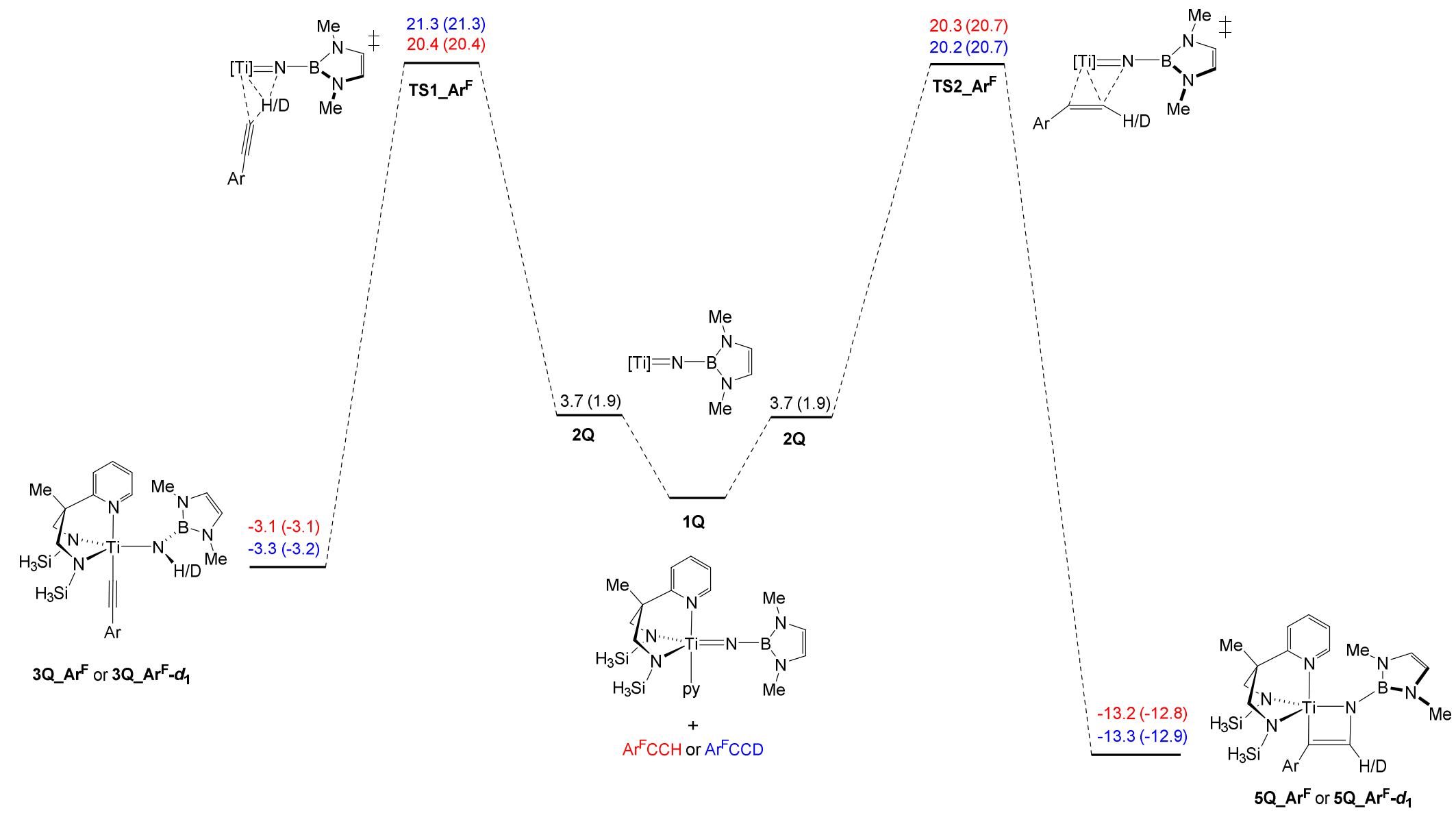

Figure S9. Relative Gibbs free energies $\left(\mathrm{G}_{\text {rel }}, \mathrm{kcal} \mathrm{mol}^{-1}\right)$ at $298 \mathrm{~K}\left(\mathrm{G}_{\text {rel }}\right.$ values at $343 \mathrm{~K}$ in parentheses) for the DFT computed, gas phase reactions of $\mathrm{Ti}\left(\mathrm{N}_{2}{ }^{\mathrm{SiH}_{3}} \mathrm{~N}^{\mathrm{py}}\right)\left\{\mathrm{NB}(\mathrm{NMeCH})_{2}\right\}\left(\right.$ py) (1Q) with $\mathrm{Ar}^{\mathrm{F}} \mathrm{CCH}$ and $\mathrm{Ar}^{\mathrm{F}} \mathrm{CCD}$. 


\section{Experimental Details}

General methods and instrumentation. All manipulations were carried out using standard Schlenk line or dry-box techniques under an atmosphere of argon or dinitrogen. Solvents were either degassed by sparging with dinitrogen and dried by passing through a column of the appropriate drying agent ${ }^{1}$ or refluxed over sodium (toluene), potassium (THF), $\mathrm{Na} / \mathrm{K}$ alloy $\left(\mathrm{Et}_{2} \mathrm{O}\right)$ or $\mathrm{CaH}_{2}$ (pyridine) and distilled. Deuterated solvents were dried over potassium $\left(\mathrm{C}_{6} \mathrm{D}_{6}\right)$ or $\mathrm{CaH}_{2}$ (pyridine- $\left.d_{5}\right)$, distilled under reduced pressure and stored under argon in Teflon valve ampoules. Unless otherwise stated NMR samples were prepared under dinitrogen in $5 \mathrm{~mm}$ Wilmad 507-PP tubes fitted with J. Young Teflon valves. ${ }^{1} \mathrm{H},{ }^{13} \mathrm{C}\left\{{ }^{1} \mathrm{H}\right\},{ }^{11} \mathrm{~B}\left\{{ }^{1} \mathrm{H}\right\}$ and ${ }^{2} \mathrm{H}$ spectra were recorded on a Bruker Ascend 400 NMR spectrometer, a Bruker Avance III 500 NMR spectrometer or on a Bruker AVC 500 spectrometer fitted with a ${ }^{13} \mathrm{C}$ cryoprobe. Unless otherwise stated, all NMR spectra were recorded at $298 \mathrm{~K} .{ }^{1} \mathrm{H}$ and ${ }^{13} \mathrm{C}\left\{{ }^{1} \mathrm{H}\right\}$ and spectra were referenced internally to residual protio-solvent $\left({ }^{1} \mathrm{H}\right)$ or solvent $\left({ }^{13} \mathrm{C}\right)$ resonances, and are reported relative to tetramethylsilane $(\delta=0 \mathrm{ppm}) .{ }^{2} \mathrm{H}$ NMR spectra were referenced to the natural abundance deuterium resonance of the protio solvent. ${ }^{11} \mathrm{~B}$ and ${ }^{19} \mathrm{~F}$ NMR spectra were referenced externally to $\mathrm{Et}_{2} \mathrm{O} \cdot \mathrm{BF}_{3}$ and $\mathrm{CFCl}_{3}$ respectively. Assignments were confirmed as necessary with the use of two dimensional ${ }^{1} \mathrm{H}-{ }^{1} \mathrm{H},{ }^{13} \mathrm{C}-{ }^{1} \mathrm{H}$ and ${ }^{19} \mathrm{~F}-{ }^{19} \mathrm{~F}$ correlation experiments. Chemical shifts are quoted in $\delta(\mathrm{ppm})$ and coupling constants in Hz. IR spectra were recorded on a Thermo Scientific Nicolet iS5 FTIR spectrometer and samples prepared in a dry-box using $\mathrm{NaCl}$ plates as a Nujol mull. The data are quoted in wavenumbers $\left(\mathrm{cm}^{-1}\right)$. Mass spectra were recorded by the mass spectrometry service of Oxford University's Department of Chemistry. Elemental analyses were carried out by the Elemental Analysis Service at the London Metropolitan University.

Starting materials. $\mathrm{Ti}\left\{\mathrm{NB}\left(\mathrm{NAr}{ }^{\prime} \mathrm{CH}\right)_{2}\right\} \mathrm{Cl}_{2}(\mathrm{py})_{3}(\mathbf{3}),{ }^{2} \mathrm{Li}_{2} \mathrm{~N}_{2}{ }^{\mathrm{SiMe}_{3}} \mathrm{~N}^{\mathrm{Me}},{ }^{3} \mathrm{H}_{2} \mathrm{~N}_{2}{ }^{\mathrm{ArF}} \mathrm{N}^{\mathrm{Me}},{ }^{4} \mathrm{Li}_{2} \mathrm{~N}_{2}{ }^{\mathrm{iPr}} \mathrm{N}^{\mathrm{Me}} 5$ $\mathrm{Li}_{2} \mathrm{~N}_{2} \mathrm{~N}^{\text {py }},{ }^{6} \mathrm{Ar}^{\mathrm{F}} \mathrm{CCH},{ }^{7} \mathrm{Ti}\left(\mathrm{N}_{2}{ }^{\mathrm{pyr}} \mathrm{N}^{\mathrm{Me}}\right)\left\{\mathrm{NB}\left(\mathrm{NAr}{ }^{\prime} \mathrm{CH}\right)_{2}\right\}(\text { py })_{2}(4)^{2}$ and $\mathrm{H}_{2} \mathrm{NB}\left(\mathrm{NAr}{ }^{\prime} \mathrm{CH}\right)_{2}{ }^{8}$ were synthesised according to literature procedures. All other reagents were purchased from commercial suppliers, and (for liquid reagents) degassed before use. TolCCH, MeCCMe and $\mathrm{PhCCMe}$ were dried over $\mathrm{CaH}_{2}$ and distilled (trap-to-trap under static vacuum) before use.

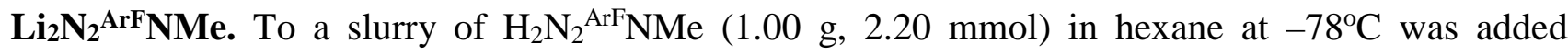
dropwise ${ }^{\mathrm{n}} \mathrm{BuLi}$ (1.6 M in hexane, $3.03 \mathrm{ml}, 4.8 \mathrm{mmol}$ ) over 10 minutes. The solution was allowed to warm to room temperature, where upon a white precipitate formed, and the solid product was then filtered and washed with hexane $(3 \times 10 \mathrm{ml})$ and dried in vacuo. Yield: $0.730 \mathrm{~g}(72 \%)$. ${ }^{1} \mathrm{H}$ NMR (pyridine- $\left.d_{5}, 299.9 \mathrm{MHz}\right): 4.18\left(4 \mathrm{H}\right.$, br. s, $\left.\mathrm{CH}_{2}\right), 2.75$ (4H, br. s, $\left.\mathrm{CH}_{2}\right), 2.01(3 \mathrm{H}, \mathrm{s}, \mathrm{NMe}) .{ }^{13} \mathrm{C}\left\{{ }^{1} \mathrm{H}\right\}$ 
(pyridine- $\left.d_{5}, 75.4 \mathrm{MHz}\right)$ : $140.0\left(o-\mathrm{C}_{6} \mathrm{~F}_{5}\right), 139.3\left(m-\mathrm{C}_{6} \mathrm{~F}_{5}\right), 124.3\left(p-\mathrm{C}_{6} \mathrm{~F}_{5}\right), 122.8\left(i-\mathrm{C}_{6} \mathrm{~F}_{5}\right), 58.1\left(\mathrm{CH}_{2}\right)$, $47.4\left(\mathrm{CH}_{2}\right), 42.1$ (NMe). ${ }^{19} \mathrm{~F}$ NMR (pyridine- $\left.d_{5}, 282.1 \mathrm{MHz}\right) ;-170.89$ (4F, br. s, $o-\mathrm{C}_{6} \mathrm{~F}_{5}$ ), -171.50 $\left(4 \mathrm{~F}, \mathrm{t},{ }^{3} J=23.8 \mathrm{~Hz}, m-\mathrm{C}_{6} \mathrm{~F}_{5}\right),-200.30\left(2 \mathrm{~F}, \mathrm{~m}, p-\mathrm{C}_{6} \mathrm{~F}_{5}\right.$ ). IR ( $\mathrm{NaCl}$ plates, Nujol mull, $\left.\mathrm{cm}^{-1}\right): 2725$ (w), 2678 (w), 2608 (w), 1662 (w), 1648 (m), 1589 (w), 1515 (s), 1360 (s), 1343 (s), 1304 (w), 1207 (w), $1174(\mathrm{~m}), 1137(\mathrm{w}), 1062$ (m), 1045 (m), 973 (s), $904(\mathrm{w}), 861(\mathrm{w}), 762(\mathrm{w}), 655(\mathrm{w}), 606(\mathrm{w})$, 529(w). Anal. found (calcd. for $\mathrm{C}_{17} \mathrm{H}_{11} \mathrm{~N}_{3} \mathrm{~F}_{10} \mathrm{Li}_{2}$ ); C, 44.10 (44.28); H, 2.60 (2.40); N, 9.00 (9.11) \%

Procedure for deuteration of $\mathrm{Ar}^{\mathrm{F}} \mathbf{C C H}$. To a stirred $\mathrm{CH}_{2} \mathrm{Cl}_{2}(5 \mathrm{~mL})$ solution of $\mathrm{Ar}^{\mathrm{F}} \mathrm{CCH}(1.00 \mathrm{~g}$, $5.21 \mathrm{mmol})$ was added $\mathrm{D}_{2} \mathrm{O}(30 \mathrm{~mL})$ and $\mathrm{NaOD}\left(40 \%\right.$ solution in $\left.\mathrm{D}_{2} \mathrm{O}, 0.020 \mathrm{~mL}, 0.27 \mathrm{mmol}\right)$. The resultant mixture was left to stir for 3 days and then quenched with $\mathrm{DCl}\left(45 \%\right.$ solution in $\mathrm{D}_{2} \mathrm{O}$, $0.028 \mathrm{~mL}$ ). The two layers were then separated and the aqueous layer was extracted with $\mathrm{CH}_{2} \mathrm{Cl}_{2}$ (2 $\times 10 \mathrm{~mL}$ ). The combined organic layers were dried over $\mathrm{Na}_{2} \mathrm{SO}_{4}$ and then the $\mathrm{CH}_{2} \mathrm{Cl}_{2}$ was removed under reduced pressure. Yield: $0.800 \mathrm{~g}(79 \%) .{ }^{1} \mathrm{H}$ NMR analysis indicated $\geq 98 \%$ deuteration.

$\mathbf{T i}\left(\mathbf{N}_{2}{ }^{\text {ArF }} \mathbf{N}^{\mathrm{Me}}\right)\left\{\mathbf{N B}\left(\mathbf{N A r} \mathbf{r}^{\prime} \mathbf{C H}\right)_{2}\right\}(\mathbf{p y})(\mathbf{1 0})$. To a Schlenk flask containing $\operatorname{Ti}\left\{\mathrm{NB}\left(\mathrm{NAr}^{\prime} \mathrm{CH}\right)_{2}\right\} \mathrm{Cl}_{2}(\mathrm{py})_{3}$ $(3,1.50 \mathrm{~g}, 1.98 \mathrm{mmol})$ and $\mathrm{Li}_{2} \mathrm{~N}_{2}{ }^{\mathrm{ArF}} \mathrm{N}^{\mathrm{Me}}(0.913 \mathrm{~g}, 1.98 \mathrm{mmol})$, was added toluene $(20 \mathrm{~mL})$ at $-78{ }^{\circ} \mathrm{C}$. The mixture was allowed to warm to room temperature and then stirred for 1 hour, after which it had become a dark red suspension. The volatiles were removed under reduced pressure, and the product extracted into pentane $(3 \times 15 \mathrm{~mL})$. The solvent was removed from the combined extracts under reduced pressure, yielding $\mathbf{1 0}$ as a deep red solid, which was then dried in vacuo. Yield: $1.51 \mathrm{~g}$ (78\%). Diffraction-quality crystals were grown from a concentrated pentane solution at $5{ }^{\circ} \mathrm{C} .{ }^{1} \mathrm{H}$ NMR $\left(\mathrm{C}_{6} \mathrm{D}_{6}, 400.1 \mathrm{MHz}\right): \delta 7.88(2 \mathrm{H}, \mathrm{m}, 2,6-\mathrm{py}), 7.18-7.10(6 \mathrm{H}$, overlapping $2 \times \mathrm{m}$ with residual protio solvent resonance, $m$ - and $\left.p-\mathrm{C}_{6} \underline{\mathrm{H}}_{3}{ }^{\mathrm{i}} \mathrm{Pr}_{2}\right), 6.46$ (1 H, m, 3,5-py), 6.09 (2 H, m, 4-py), 5.79 (2 H, s, $\mathrm{NCH}), 3.60$ (4 H, sept., $\left.{ }^{3} \mathrm{~J}=6.9 \mathrm{~Hz}, \mathrm{CHMeMe}\right), 3.47\left(2 \mathrm{H}, \mathrm{m}, \underline{\mathrm{C}}_{2} \mathrm{NAr}{ }^{\mathrm{F}}\right), 3.20\left(2 \mathrm{H}, \mathrm{m}, \mathrm{C}_{2} \mathrm{NAr}^{\mathrm{F}}\right)$, $2.49\left(2 \mathrm{H}, \mathrm{m}, \mathrm{C}_{2} \mathrm{NMe}\right), 2.28\left(2 \mathrm{H}, \mathrm{m}, \underline{\mathrm{C}}_{2} \mathrm{NMe}\right), 1.96(3 \mathrm{H}, \mathrm{s}, \mathrm{NMe}), 1.28\left(12 \mathrm{H}, \mathrm{d},{ }^{3} \mathrm{~J}=6.9 \mathrm{~Hz}\right.$, $\mathrm{CHMeMe}), 1.26\left(12 \mathrm{H}, \mathrm{d},{ }^{3} J=6.9 \mathrm{~Hz}, \mathrm{CHMeMe}\right) \mathrm{ppm} .{ }^{13} \mathrm{C}\left\{{ }^{1} \mathrm{H}\right\} \mathrm{NMR}\left(\mathrm{C}_{6} \mathrm{D}_{6}, 100.6 \mathrm{MHz}\right): \delta 151.4$ (2,6-py), $147.6\left(i-\underline{\mathrm{C}}_{6} \mathrm{H}_{3}{ }^{\mathrm{i}} \mathrm{Pr}_{2}\right), 144.4\left(o-\mathrm{C}_{6} \mathrm{~F}_{5}\right), 141.4\left(o-\underline{\mathrm{C}}_{6} \mathrm{H}_{3}{ }^{\mathrm{i}} \mathrm{Pr}_{2}\right), 139.5$ ( $\left(m-\mathrm{C}_{6} \mathrm{~F}_{5}\right), 138.3$ (4-py), 137.0

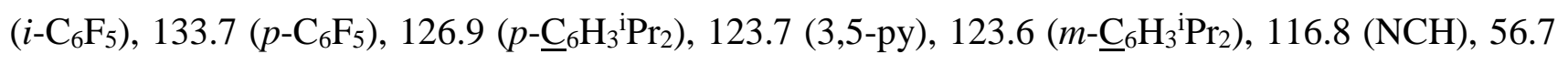

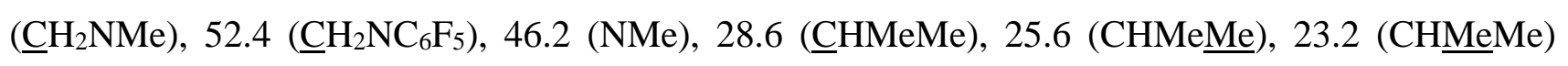
ppm. ${ }^{11} \mathrm{~B}\left\{{ }^{1} \mathrm{H}\right\}$ NMR $\left(\mathrm{C}_{6} \mathrm{D}_{6}, 128.4 \mathrm{MHz}\right): \delta 14.7 \mathrm{ppm} .{ }^{19} \mathrm{~F}\left\{{ }^{1} \mathrm{H}\right\}$ NMR $\left(\mathrm{C}_{6} \mathrm{D}_{6}, 376.5 \mathrm{MHz}\right): \delta-149.4(2$ $\left.\mathrm{F}, \mathrm{d},{ }^{3} J=22.4 \mathrm{~Hz}, o-\mathrm{C}_{6} \mathrm{~F}_{5}\right),-166.0\left(2 \mathrm{~F}\right.$, app. t, app. $\left.{ }^{3} J=22.4 \mathrm{~Hz}, m-\mathrm{C}_{6} \mathrm{~F}_{5}\right),-169.9\left(1 \mathrm{~F}, \mathrm{t},{ }^{3} J=22.4\right.$ $\left.\mathrm{Hz}, p-\mathrm{C}_{6} \mathrm{~F}_{5}\right)$ ppm. IR (NaCl plates, Nujol mull, $\left.\mathrm{cm}^{-1}\right): 3084(\mathrm{w}), 2608$ (w), $2459(\mathrm{w}), 1997(\mathrm{w}), 1605$ (w), 1583 (m), 1507 (m), 1491 (m), 1446 (w), 1406 (m), 1368 (s), 1303 (m), 1259 (m), 1157 (m), $1114(\mathrm{w}), 1086$ (w), 1065 (m), 982 (s), 931 (w), 897 (w), 862 (m), 806 (w), 765 (m), 752 (w), 702 
(m), $668(w), 640(w)$. EI-MS: $m / z=896[M-\text { py }]^{+}(4 \%)$. Anal. found (calcd. for $\mathrm{C}_{48} \mathrm{H}_{52} \mathrm{BF}_{10} \mathrm{~N}_{7} \mathrm{Ti}$ ): C, 59.17 (59.09); H, 5.36 (5.37); N, 9.98 (10.05)\%.

$\mathbf{T i}\left(\mathbf{N}_{2}{ }^{i P r} \mathbf{N}^{M e}\right)\left\{\mathbf{N B}\left(\mathbf{N A r} \mathbf{C H}^{\prime} \mathbf{C H}\right)_{2}\right\}$ (py) (11). To a Schlenk flask containing $\mathrm{Ti}\left\{\mathrm{NB}\left(\mathrm{NAr}^{\prime} \mathrm{CH}\right)_{2}\right\} \mathrm{Cl}_{2}(\text { py })_{3}$ $(3,1.50 \mathrm{~g}, 1.98 \mathrm{mmol})$ and $\mathrm{Li}_{2} \mathrm{~N}_{2}{ }^{\mathrm{iPr}} \mathrm{N}^{\mathrm{Me}}(0.422 \mathrm{~g}, 1.98 \mathrm{mmol})$ was added toluene $(30 \mathrm{~mL})$ at $-78{ }^{\circ} \mathrm{C}$. The mixture was allowed to warm to RT, and then stirred for $3 \mathrm{~h}$, after which time it had become a deep red suspension. The volatiles were removed under reduced pressure, and the product extracted into pentane $(4 \times 15 \mathrm{~mL})$. The solvent was removed from the combined extracts under reduced pressure, resulting in $\mathbf{1 1}$ as a red solid, which was then dried in vacuo. Yield: $1.27 \mathrm{~g}$ (88\%). Diffraction-quality crystals were grown from a concentrated pentane solution at $5{ }^{\circ} \mathrm{C} .{ }^{1} \mathrm{H}$ NMR $\left(\mathrm{C}_{6} \mathrm{D}_{6}, 400.1 \mathrm{MHz}\right): \delta 8.01(2 \mathrm{H}, \mathrm{m}, 2,6-p y), 7.25$ (6 H, overlapping $2 \times \mathrm{m}, m$ - and $\left.p-\mathrm{C}_{6} \underline{\mathrm{H}}_{3}{ }^{\mathrm{i}} \mathrm{Pr}_{2}\right), 6.76$ (1 H, m, 4-py), $6.40(2 \mathrm{H}, \mathrm{m}, 3,5-\mathrm{py}), 5.90(2 \mathrm{H}, \mathrm{s}, \mathrm{NCH}), 3.87$ (4 H, sept., ${ }^{3} J=6.9 \mathrm{~Hz}$, $\left.\mathrm{C}_{6} \mathrm{H}_{3}(\mathrm{C} \underline{\mathrm{HMeMe}})_{2}\right), 3.76$ (2 H, sept., $\left.{ }^{3} \mathrm{~J}=6.5 \mathrm{~Hz}, \mathrm{NC} \underline{\mathrm{HMeMe}}\right) 3.29$ (2 H, m, $\underline{\mathrm{H}}_{2} \mathrm{~N}$ Pr), 3.08 (2 H, m, $\left.\mathrm{C}_{2} \mathrm{~N}^{\mathrm{i}} \mathrm{Pr}\right), 2.77\left(2 \mathrm{H}, \mathrm{m}, \mathrm{C}_{2} \mathrm{NMe}\right), 2.29\left(2 \mathrm{H}, \mathrm{m}, \mathrm{C}_{2}{ }_{2} \mathrm{NMe}\right), 1.55(3 \mathrm{H}, \mathrm{s}, \mathrm{NMe}), 1.50\left(12 \mathrm{H}, \mathrm{d},{ }^{3} \mathrm{~J}=\right.$ $\left.6.9 \mathrm{~Hz}, \mathrm{C}_{6} \mathrm{H}_{3}(\mathrm{CHMeMe})_{2}\right), 1.41\left(6 \mathrm{H}, \mathrm{d},{ }^{3} J=6.5 \mathrm{~Hz}, \mathrm{NCHMeMe}\right), 1.37\left(12 \mathrm{H}, \mathrm{d},{ }^{3} J=6.9 \mathrm{~Hz}\right.$, $\left.\mathrm{C}_{6} \mathrm{H}_{3}(\mathrm{CHMeMe})_{2}\right), 0.66\left(6 \mathrm{H}, \mathrm{d},{ }^{3} J=6.5 \mathrm{~Hz}, \mathrm{NCHMeMe}\right) \mathrm{ppm} .{ }^{13} \mathrm{C}\left\{{ }^{1} \mathrm{H}\right\} \mathrm{NMR}\left(\mathrm{C}_{6} \mathrm{D}_{6}, 100.6 \mathrm{MHz}\right)$ :

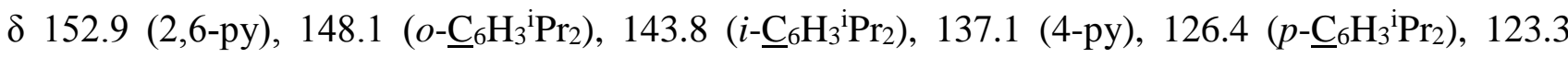
(overlapping $m-\underline{\mathrm{C}}_{6} \mathrm{H}_{3}{ }^{\mathrm{i}} \mathrm{Pr}_{2}$ and 3,5-py), $116.8(\mathrm{NCH}), 58.7\left(\underline{\mathrm{CH}}_{2} \mathrm{NMe}\right), 51.4$ (NCHMeMe), 45.1 $\left.\left(\underline{\mathrm{CH}_{2}} \mathrm{~N}^{\mathrm{i}} \mathrm{Pr}\right), 45.0(\mathrm{NMe}), 28.7\left(\mathrm{C}_{6} \mathrm{H}_{3}(\underline{\mathrm{CHMeMe}})_{2}\right), 25.2\left(\mathrm{C}_{6} \mathrm{H}_{3}(\mathrm{CHMeMe})\right)_{2}\right), 24.3\left(\mathrm{C}_{6} \mathrm{H}_{3}(\mathrm{CHMeMe})_{2}\right)$, 21.5 (NCHMeMe), 21.1 (NCHMeMe) ppm. ${ }^{11} \mathrm{~B}\left\{{ }^{1} \mathrm{H}\right\} \mathrm{NMR}\left(\mathrm{C}_{6} \mathrm{D}_{6}, 128.4 \mathrm{MHz}\right): \delta 15.2 \mathrm{ppm} . \mathrm{IR}$ ( $\mathrm{NaCl}$ plates, Nujol mull, cm ${ }^{-1}$ ): 2777 (w), 2653 (w), 1601 (s), 1543 (w), 1443 (w), 1422 (m), 1359 (s), 1350 (m), 1337 (s), 1308 (m), 1287 (w), 1272 (w), 1239 (w), 1210 (m), 1179 (w), 1139 (w), 1065 (m), 1040 (w), 1013 (w), 999 (m), 930 (w), 895 (w), 879 (w), 814 (w), 763 (m), 755 (w), 699 (m), 668 (m), 640 (m). Anal. found (calcd. for $\left.\mathrm{C}_{42} \mathrm{H}_{66} \mathrm{BN}_{7} \mathrm{Ti}\right)$ : C, 69.23 (69.32); H, 9.31 (9.14); N, $13.55(13.47) \%$.

$\operatorname{Ti}\left(\mathbf{N}_{2}{ }^{\text {SiMe3 }} \mathbf{N}^{M e}\right)\left\{\mathbf{N}\left\{\mathbf{B}\left(\mathbf{N A r} \mathbf{C H}^{\prime} \mathbf{C H}\right)_{2} \mathbf{C}(\mathbf{H}) \mathbf{C}\left(\mathbf{A r}^{\mathrm{F}}\right)\right\} \quad\right.$ (13). $\quad$ To $\quad$ a $\quad$ solution of $\mathrm{Ti}\left(\mathrm{N}_{2}{ }^{\mathrm{SiMe} 3} \mathrm{~N}^{\mathrm{Me}}\right)\left\{\mathrm{NB}\left(\mathrm{NAr}{ }^{\prime} \mathrm{CH}\right)_{2}\right\}\left(\right.$ py) $(\mathbf{9}, 0.261 \mathrm{~g}, 0.331 \mathrm{mmol})$ in hexane $(15 \mathrm{~mL})$ was added $\mathrm{Ar}^{\mathrm{F}} \mathrm{CCH}$ (44 $\mu \mathrm{L}, 0.331 \mathrm{mmol}$ ) at $\mathrm{RT}$, giving an immediate color change to deep red/brown. The reaction mixture was stirred at RT for $1 \mathrm{~h}$, after which the volatiles were removed under reduced pressure, giving 13 as a deep brown waxy solid. Yield: $0.198 \mathrm{~g}(66 \%) .{ }^{1} \mathrm{H}$ NMR $\left(\mathrm{C}_{6} \mathrm{D}_{6}, 400.1 \mathrm{MHz}\right): \delta 10.43$ (br. s overlapping with minor isomer, $\mathrm{TiC}=\mathrm{CH}$ ), $7.22-7.11$ (overlapping $2 \times \mathrm{m}$ with minor isomer and residual protio solvent resonance, $m$ - and $\left.p-\mathrm{C}_{6} \underline{\mathrm{H}}_{3}{ }^{\mathrm{i}} \operatorname{Pr}_{2}\right), 6.11(2 \mathrm{H}, \mathrm{s}, \mathrm{NCH}), 3.39\left(4 \mathrm{H}\right.$, sept., ${ }^{3} J=$ $6.9 \mathrm{~Hz}, \mathrm{C} \underline{\mathrm{HMeMe}}$ ), 3.02 (2 H, m, $\left.\underline{\mathrm{H}}_{2} \mathrm{NSiMe}_{3}\right), 2.62$ (2 H, m, $\left.\underline{\mathrm{H}}_{2} \mathrm{NMe}\right), 1.97$ (3 H, s, NMe), 1.89 (2 $\left.\mathrm{H}, \mathrm{m}, \underline{\mathrm{CH}}_{2} \mathrm{NMe}\right), 1.29\left(12 \mathrm{H}, \mathrm{d},{ }^{3} \mathrm{~J}=6.9 \mathrm{~Hz}, \mathrm{CH} \underline{\mathrm{MeMe}}\right), 1.20\left(12 \mathrm{H}, \mathrm{d},{ }^{3} \mathrm{~J}=6.9 \mathrm{~Hz}, \mathrm{CHMeMe}\right) 0.02$ 
(18 H, s, $\mathrm{SiMe}_{3}$ ) ppm. A second resonance assigned to $\underline{\mathrm{C}}_{2} \mathrm{NSiMe}_{3}$ is found to be obscured by the C $\underline{H M e M e}$ resonance at $3.39 \mathrm{ppm}$, as observed by HSQC and HMBC spectra. ${ }^{13} \mathrm{C}\left\{{ }^{1} \mathrm{H}\right\} \mathrm{NMR}\left(\mathrm{C}_{6} \mathrm{D}_{6}\right.$, $125.7 \mathrm{MHz}): \delta 179.4(\mathrm{TiC}=\mathrm{CH}), 161.8(\mathrm{TiC}=\underline{\mathrm{CH}}), 147.2\left(o-\underline{\mathrm{C}}_{6} \mathrm{H}_{3}{ }^{\mathrm{i}} \mathrm{Pr}_{2}\right), 143.7\left(\mathrm{~m}, o-\mathrm{C}_{6} \mathrm{~F}_{5}\right), 141.8(\mathrm{~m}$, $\left.m-\mathrm{C}_{6} \mathrm{~F}_{5}\right), 139.5\left(i-\underline{\mathrm{C}}_{6} \mathrm{H}_{3}{ }^{\mathrm{i}} \mathrm{Pr}_{2}\right), 136.5\left(\mathrm{~m}, p-\mathrm{C}_{6} \mathrm{~F}_{5}\right), 124.2\left(m-\underline{\mathrm{C}}_{6} \mathrm{H}_{3}{ }^{\mathrm{i}} \mathrm{Pr}_{2}\right), 121.2\left(\mathrm{~m}, i-\mathrm{C}_{6} \mathrm{~F}_{5}\right), 119.2(\mathrm{NCH})$, $57.8\left(\underline{\mathrm{CH}}_{2} \mathrm{NMe}\right), 51.0 \quad\left(\underline{\mathrm{CH}}_{2} \mathrm{NSiMe}_{3}\right), 43.3 \quad(\mathrm{NMe}), 29.0$ (대MeMe), 26.3 (CHMeMe), 23.7 (CHMeMe), $1.9\left(\mathrm{SiMe}_{3}\right)$ ppm. The $p-\underline{\mathrm{C}}_{6} \mathrm{H}_{3}{ }^{\mathrm{i}} \mathrm{Pr}_{2}$ resonance is obscured by the solvent resonance. ${ }^{19} \mathrm{~F}\left\{{ }^{1} \mathrm{H}\right\}$ NMR $\left(\mathrm{C}_{6} \mathrm{D}_{6}, 376.5 \mathrm{MHz}\right):-140.4\left(2 \mathrm{~F}, \mathrm{~d},{ }^{3} J=21.6 \mathrm{~Hz}, o-\mathrm{C}_{6} \mathrm{~F}_{5}\right),-163.1\left(1 \mathrm{~F}, \mathrm{t},{ }^{3} J=21.5\right.$ $\left.\mathrm{Hz}, p-\mathrm{C}_{6} \mathrm{~F}_{5}\right),-165.4\left(2 \mathrm{~F}, \mathrm{~m}, m-\mathrm{C}_{6} \mathrm{~F}_{5}\right) \mathrm{ppm} .{ }^{11} \mathrm{~B}\left\{{ }^{1} \mathrm{H}\right\} \mathrm{NMR}\left(\mathrm{C}_{6} \mathrm{D}_{6}, 128.4 \mathrm{MHz}\right): \delta 23.4 \mathrm{ppm}$. IR $(\mathrm{NaCl}$ plates, Nujol mull, cm²): 3522 (w), 3391 (m), 1832 (w), 1866 (w), 1797 (w), 1655 (m), 1637 (m), 1586 (m), 1510 (m), 1201 (m), 1180 (m), 1117 (s), 1031 (s), 960 (s), 909 (s), 837 (s), 759 (m), 674 (m), $651(\mathrm{~m}), 604(\mathrm{~m}), 556(\mathrm{~m})$. EI-MS: $m / z=595\left[\mathrm{Ar}^{\mathrm{F}} \mathrm{C}(\mathrm{H}) \mathrm{C}(\mathrm{H}) \mathrm{N}(\mathrm{H}) \mathrm{B}\left(\mathrm{NAr}{ }^{\prime} \mathrm{CH}\right)_{2}\right]^{+}(100 \%)$. Anal. found (calcd. for $\mathrm{C}_{45} \mathrm{H}_{66} \mathrm{BF}_{5} \mathrm{~N}_{6} \mathrm{Si}_{2} \mathrm{Ti}$ ): C, 59.71 (59.99); H, 7.13 (7.38); N, 9.32 (9.33)\%.

$\operatorname{Ti}\left(\mathbf{N}_{2}{ }^{A r F} N^{M e}\right)\left\{\mathbf{N}\left\{B\left(N_{A r} \mathbf{C H}\right)_{2}\right\} \mathbf{C}(\mathbf{H}) \mathbf{C}(\mathbf{T o l})\right\} \quad$ (14). To $\quad$ To solution of $\mathrm{Ti}\left(\mathrm{N}_{2}{ }^{\mathrm{ArF}} \mathrm{N}^{\mathrm{Me}}\right)\left\{\mathrm{NB}\left(\mathrm{NAr} \mathrm{CH}_{2}\right\}(\mathrm{py})(\mathbf{1 0}, 0.499 \mathrm{~g}, 0.511 \mathrm{mmol})\right.$ in toluene $(10 \mathrm{~mL})$ was added TolCCH $(65 \mu \mathrm{L}, 0.511 \mathrm{mmol})$ at RT, giving an immediate color change to deep red. The reaction mixture was stirred for 20 minutes, after which the volatiles were removed under reduced pressure, to yield $\mathbf{1 4}$ as a deep red solid, which was then dried in vacuo. Yield: $0.395 \mathrm{~g}$ (76\%). Diffraction-quality crystals were grown from a concentrated hexane solution at $5{ }^{\circ} \mathrm{C} .{ }^{1} \mathrm{H} \mathrm{NMR}\left(\mathrm{C}_{6} \mathrm{D}_{6}, 400.1 \mathrm{MHz}\right): \delta 11.61(1 \mathrm{H}$, $\mathrm{s}, \mathrm{TiC}=\mathrm{CH}), 7.27-7.19\left(6 \mathrm{H}\right.$, overlapping $2 \times \mathrm{m}, m-$ and $\left.p-\mathrm{C}_{6} \underline{\mathrm{H}}_{3}{ }^{\mathrm{i}} \operatorname{Pr}_{2}\right), 6.65\left(2 \mathrm{H}, \mathrm{d},{ }^{3} J=7.9 \mathrm{~Hz}, o-\right.$ $\left.\mathrm{C}_{6} \underline{\mathrm{H}}_{4} \mathrm{Me}\right), 6.12\left(2 \mathrm{H}, \mathrm{d},{ }^{3} \mathrm{~J}=7.9 \mathrm{~Hz}, m-\mathrm{C}_{6} \underline{\mathrm{H}}_{4} \mathrm{Me}\right), 6.01(2 \mathrm{H}, \mathrm{s}, \mathrm{NCH}), 3.67\left(2 \mathrm{H}, \mathrm{m}, \mathrm{CH}_{2} \mathrm{NAr}^{\mathrm{F}}\right), 3.49$ (4 H, sept., $\left.{ }^{3} J=6.9 \mathrm{~Hz}, \mathrm{C} \underline{\mathrm{HMeMe}}\right), 3.10\left(2 \mathrm{H}, \mathrm{m}, \mathrm{CH}_{2} \mathrm{NAr}^{\mathrm{F}}\right), 2.95\left(2 \mathrm{H}, \mathrm{m}, \mathrm{C}_{2} \mathrm{NMe}\right), 2.19(2 \mathrm{H}, \mathrm{m}$, $\left.\underline{\mathrm{C}}_{2} \mathrm{NMe}\right), 1.86$ (3 H, s, $\left.\mathrm{C}_{6} \mathrm{H}_{4} \underline{\mathrm{Me}}\right), 1.28\left(24 \mathrm{H}\right.$, app. t, app. $\left.{ }^{3} \mathrm{~J}=7.5 \mathrm{~Hz}, \mathrm{CHMeMe}\right), 1.27$ (3 H, s, NMe) ppm. ${ }^{13} \mathrm{C}\left\{{ }^{1} \mathrm{H}\right\}$ NMR $\left(\mathrm{C}_{6} \mathrm{D}_{6}, 125.8 \mathrm{MHz}\right): \delta 217.2(\mathrm{TiC}=\mathrm{CH}), 163.8(\mathrm{TiC}=\underline{\mathrm{C}} \mathrm{H}), 147.5$ $\left(o-\underline{\mathrm{C}}_{6} \mathrm{H}_{3}{ }^{\mathrm{i}} \mathrm{Pr}_{2}\right), 143.3\left(\mathrm{~m}, o-\mathrm{C}_{6} \mathrm{~F}_{5}\right), 141.4\left(\mathrm{~m}, p-\mathrm{C}_{6} \mathrm{~F}_{5}\right), 140.8\left(i-\underline{\mathrm{C}}_{6} \mathrm{H}_{3}{ }^{\mathrm{i}} \mathrm{Pr}_{2}\right), 137.2\left(i-\underline{\mathrm{C}}_{6} \mathrm{H}_{4} \mathrm{Me}\right), 136.7(\mathrm{~m}$, $\left.m-\mathrm{C}_{6} \mathrm{~F}_{5}\right), 135.7\left(p-\underline{\mathrm{C}}_{6} \mathrm{H}_{4} \mathrm{Me}\right), 133.3\left(\mathrm{td},{ }^{2} J_{\mathrm{C}-\mathrm{F}}=14.3 \mathrm{~Hz},{ }^{3} J_{\mathrm{C}-\mathrm{F}}=3.7 \mathrm{~Hz}, i-\mathrm{C}_{6} \mathrm{~F}_{5}\right), 129.3\left(p-\underline{\mathrm{C}}_{6} \mathrm{H}_{3}{ }^{\mathrm{i}} \operatorname{Pr}_{2}\right)$, $128.6\left(m-\underline{\mathrm{C}}_{6} \mathrm{H}_{4} \mathrm{Me}\right), 126.3\left(o-\underline{\mathrm{C}}_{6} \mathrm{H}_{4} \mathrm{Me}\right), 124.5\left(m-\underline{\mathrm{C}}_{6} \mathrm{H}_{3}{ }^{\mathrm{i}} \mathrm{Pr}_{2}\right), 119.2(\mathrm{NCH}), 56.8\left(\underline{\mathrm{CH}}_{2} \mathrm{NAr}{ }^{\mathrm{F}}\right), 55.9$ ( $\left.\underline{\mathrm{CH}}_{2} \mathrm{NMe}\right), 41.4$ (NMe), 29.0 (매MeMe), 25.0 (CHMeMe), 23.4 (CHMe $\left.\underline{\mathrm{CHe}}\right), 20.8\left(\mathrm{C}_{6} \mathrm{H}_{4} \underline{\mathrm{Me}}\right) \mathrm{ppm}$. ${ }^{11} \mathrm{~B}\left\{{ }^{1} \mathrm{H}\right\}$ NMR $\left(\mathrm{C}_{6} \mathrm{D}_{6}, 128.4 \mathrm{MHz}\right): \delta 21.9 \mathrm{ppm} .{ }^{19} \mathrm{~F}\left\{{ }^{1} \mathrm{H}\right\} \mathrm{NMR}\left(\mathrm{C}_{6} \mathrm{D}_{6}, 376.5 \mathrm{MHz}\right):-150.1(2 \mathrm{~F}, \mathrm{~m}$, $\left.o-\mathrm{C}_{6} \mathrm{~F}_{5}\right),-166.1\left(2 \mathrm{~F}, \mathrm{~m}, m-\mathrm{C}_{6} \mathrm{~F}_{5}\right),-167.0\left(1 \mathrm{~F}, \mathrm{t},{ }^{3} J=21.9 \mathrm{~Hz}, p-\mathrm{C}_{6} \mathrm{~F}_{5}\right)$. IR (NaCl plates, Nujol mull, $\mathrm{cm}^{-1}$ ): 3407 (w), 3067 (w), 1942 (w), 1658 (m), 1625 (w), 1583 (m), 1496 (s), 1408 (w), 1391 (m), $1349(\mathrm{w}), 1278$ (w), 1207 (m), 1118 (w), 1065 (w), 982 (s), 937 (w), 901 (m), 861 (m), $816(\mathrm{w}), 769$ $(\mathrm{m}), 694(\mathrm{w}), 650(\mathrm{~m})$. EI-MS: $\mathrm{m} / z=1012[M]^{+}(10 \%)$. Anal. found (calcd. for $\left.\mathrm{C}_{52} \mathrm{H}_{55} \mathrm{BF}_{10} \mathrm{~N}_{6} \mathrm{Ti}\right): \mathrm{C}$, 61.54 (61.67); H, 5.65 (5.47); N, $8.12(8.30) \%$. 
NMR tube scale synthesis of $\operatorname{Ti}\left(\mathrm{N}_{2}{ }^{A r F} N^{M e}\right)\left\{\mathbf{N}\left\{B\left(N A r^{\prime} C H\right)_{2}\right\} \mathbf{C}(\mathbf{H}) \mathbf{C}\left(\mathrm{Ar}^{\mathrm{F}}\right)\right\}(16)$. To an NMR tube fitted with a J. Youngs valve was added a solution of $\operatorname{Ti}\left(\mathrm{N}_{2}{ }^{\mathrm{ArF}} \mathrm{N}^{\mathrm{Me}}\right)\left\{\mathrm{NB}\left(\mathrm{NAr}{ }^{\prime} \mathrm{CH}\right)_{2}\right\}($ py) (10, 0.0150 $\mathrm{g}, 0.0153 \mathrm{mmol})$ and $\mathrm{B}\left(\mathrm{Ar}^{\mathrm{F}}\right)_{3}(0.0078 \mathrm{~g}, 0.0153 \mathrm{mmol})$ in $\mathrm{C}_{6} \mathrm{D}_{6}(0.6 \mathrm{~mL})$. $\mathrm{Ar}^{\mathrm{F}} \mathrm{CCH}(2.0 \mu \mathrm{L}, 0.0153$ mmol) was added via microsyringe, followed by an immediate color change to very deep brown. Conversion to 16 and [py- $\left.\mathrm{B}\left(\mathrm{Ar}^{\mathrm{F}}\right)_{3}\right]$ was found to be quantitative. Major isomer: ${ }^{1} \mathrm{H}$ NMR $\left(\mathrm{C}_{6} \mathrm{D}_{6}\right.$, 400.1 MHz): $\delta 11.51(1 \mathrm{H}, \mathrm{s}, \mathrm{TiC}=\mathrm{CH}), 7.26-7.17$ (multiple $\mathrm{m}$ overlapping with minor isomer, $m$ and $\left.p-\mathrm{C}_{6} \underline{\mathrm{H}}_{3}{ }^{\mathrm{i}} \mathrm{Pr}_{2}\right), 5.98(2 \mathrm{H}, \mathrm{s}, \mathrm{NCH}), 3.46(4 \mathrm{H}$, sept. overlapping with minor isomer, $\mathrm{C} \underline{\mathrm{HMeMe}})$, 2.91 (multiple m overlapping with minor isomer, $\mathrm{CH}_{2} \mathrm{NAr}^{\mathrm{F}}$ and $\left.\underline{\mathrm{C}}_{2} \mathrm{NMe}\right), 2.12\left(2 \mathrm{H}, \mathrm{m}, \mathrm{C}_{2} \mathrm{NMe}\right)$, 1.26 (multiple m overlapping with minor isomer, $\mathrm{NMe}$ and $\mathrm{CHMeMe}) \mathrm{ppm} .{ }^{13} \mathrm{C}\left\{{ }^{1} \mathrm{H}\right\} \mathrm{NMR}\left(\mathrm{C}_{6} \mathrm{D}_{6}\right.$, 125.7 MHz): $\delta 194.1(\mathrm{Ti} \underline{\mathrm{C}}=\mathrm{CH}), 162.6(\mathrm{TiC}=\underline{\mathrm{C}} \mathrm{H}), 147.2\left(o-\underline{\mathrm{C}}_{6} \mathrm{H}_{3}{ }^{\mathrm{i}} \operatorname{Pr}_{2}\right), 140.1\left(i-\underline{\mathrm{C}}_{6} \mathrm{H}_{3}{ }^{\mathrm{i}} \operatorname{Pr}_{2}\right), 128.4(p-$ $\left.\underline{\mathrm{C}}_{6} \mathrm{H}_{3}{ }^{\mathrm{i}} \mathrm{Pr}_{2}\right), 124.9\left(m-\underline{\mathrm{C}}_{6} \mathrm{H}_{3}{ }^{\mathrm{i}} \mathrm{Pr}_{2}\right), 119.6(\mathrm{NCH}), 56.4\left(\underline{\mathrm{CH}}_{2} \mathrm{NMe}\right), 55.8\left(\underline{\mathrm{CH}}_{2} \mathrm{NAr}^{\mathrm{F}}\right), 41.6(\mathrm{NMe}), 28.9$ (CHMeMe), 25.3 (CHMeMe), 23.2 (CHMeMe) ppm. Those resonances belonging to the $\mathrm{NAr}^{\mathrm{F}}$ and $\mathrm{TiC}\left(\underline{\mathrm{Ar}^{\mathrm{F}}}\right)=\mathrm{CH}$ rings could not be satisfactorily assigned. Minor isomer: ${ }^{1} \mathrm{H} \mathrm{NMR}\left(\mathrm{C}_{6} \mathrm{D}_{6}, 400.1 \mathrm{MHz}\right)$ : $\delta 11.30(1 \mathrm{H}, \mathrm{s}, \mathrm{TiC}=\mathrm{CH}), 7.26-7.17$ (multiple $\mathrm{m}$ overlapping with major isomer, $m$ - and $p$ $\left.\mathrm{C}_{6} \underline{\mathrm{H}}_{3}{ }^{\mathrm{i}} \mathrm{Pr}_{2}\right), 6.10(2 \mathrm{H}, \mathrm{s}, \mathrm{NCH}), 3.76\left(4 \mathrm{H}, \mathrm{m}, \mathrm{CH}_{2} \mathrm{NAr}^{\mathrm{F}}\right), 3.46(4 \mathrm{H}$, sept. overlapping with major isomer, $\mathrm{C} \underline{\mathrm{HMeMe}}$ ), 2.91 (m overlapping with major isomer, $\left.\underline{\mathrm{C}}_{2} \mathrm{NMe}\right), 2.74(3 \mathrm{H}, \mathrm{s}, \mathrm{NMe}) 1.93(2$ $\mathrm{H}, \mathrm{m}, \underline{\mathrm{C}}_{2} \mathrm{NMe}$ ), 1.26 (multiple m overlapping with major isomer, $\mathrm{CHMeMe}$ ) ppm. ${ }^{13} \mathrm{C}\left\{{ }^{1} \mathrm{H}\right\} \mathrm{NMR}$ $\left(\mathrm{C}_{6} \mathrm{D}_{6}, 125.7 \mathrm{MHz}\right): \delta 201.1(\mathrm{TiC}=\mathrm{CH}), 160.2(\mathrm{TiC}=\underline{\mathrm{CH}}), 146.0\left(o-\underline{\mathrm{C}}_{6} \mathrm{H}_{3}{ }^{\mathrm{i}} \mathrm{Pr}_{2}\right), 124.3\left(m-\underline{\mathrm{C}}_{6} \mathrm{H}_{3}{ }^{\mathrm{i}} \mathrm{Pr}_{2}\right)$, $118.8(\mathrm{NCH}), 56.9\left(\underline{\mathrm{CH}}_{2} \mathrm{NMe}\right), 55.4\left(\underline{\mathrm{CH}}_{2} \mathrm{NAr}^{\mathrm{F}}\right), 37.8(\mathrm{NMe}), 29.0(\underline{\mathrm{CHMeMe}}), 25.9(\mathrm{CHMeMe})$, $23.1(\mathrm{CHMeMe})$ ppm. Those resonances belonging to the $\mathrm{NAr}^{\mathrm{F}}$ and $\operatorname{TiC}\left(\underline{\operatorname{Ar}}{ }^{\mathrm{F}}\right)=\mathrm{CH}$ rings, and the $i$ $\underline{\mathrm{C}}_{6} \mathrm{H}_{3}{ }^{\mathrm{i}} \mathrm{Pr}_{2}$ resonance could not be satisfactorily assigned. The $p-\underline{\mathrm{C}}_{6} \mathrm{H}_{3}{ }^{\mathrm{i}} \operatorname{Pr}_{2}$ resonance is obscured by the solvent resonance. Common data: ${ }^{11} \mathrm{~B}\left\{{ }^{1} \mathrm{H}\right\}$ NMR $\left(\mathrm{C}_{6} \mathrm{D}_{6}, 128.4 \mathrm{MHz}\right): \delta 22.6 \mathrm{ppm}$.

$\mathrm{Ti}\left\{\mathrm{MeN}\left(\mathrm{CH}_{2} \mathrm{CH}_{2} \mathrm{NAr}^{\mathrm{F}}\right)\left\{\mathrm{CH}_{2} \mathrm{CH}_{2} \mathrm{NC}_{6} \mathrm{~F}_{4} \mathrm{C}(\mathrm{Tol})=\mathrm{CHNB}\left(\mathrm{NAr}^{\prime} \mathrm{CH}\right)_{2}\right\}\right\}(\mathrm{F})$ (17). To a solution of $\mathrm{Ti}\left(\mathrm{N}_{2}{ }^{\mathrm{ArF}} \mathrm{N}^{\mathrm{Me}}\right)\left\{\mathrm{NB}\left(\mathrm{NAr}{ }^{\prime} \mathrm{CH}\right)_{2}\right\}($ py) $(\mathbf{1 0}, 0.290 \mathrm{~g}, 0.297 \mathrm{mmol})$ in toluene $(10 \mathrm{~mL})$ was added TolCCH (38 $\mu \mathrm{L}, 0.297 \mathrm{mmol}$ ) at $\mathrm{RT}$, giving an immediate color change to deep red. The reaction mixture was heated to $70{ }^{\circ} \mathrm{C}$ and stirred for $3.5 \mathrm{~h}$, after which the volatiles were removed under reduced pressure, resulting in an orange waxy solid. Washing with hexane $(3 \times 10 \mathrm{~mL})$ gave $\mathbf{1 7}$ as a yellow-orange solid. Yield: $0.141 \mathrm{~g}(47 \%)$. Diffraction-quality crystals were grown from a concentrated hexane solution at RT. ${ }^{1} \mathrm{H}$ NMR $\left(\mathrm{C}_{6} \mathrm{D}_{6}, 400.1 \mathrm{MHz}\right): \delta 7.31\left(2 \mathrm{H}, \mathrm{t},{ }^{3} J=7.7 \mathrm{~Hz}, p-\mathrm{C}_{6} \underline{\mathrm{H}}_{3}{ }^{\mathrm{i}} \mathrm{Pr}_{2}\right), 7.15\left(2 \mathrm{H}, \mathrm{d},{ }^{3} J\right.$ $\left.=7.7 \mathrm{~Hz}, m_{\mathrm{a}}-\mathrm{C}_{6} \underline{\mathrm{H}}_{3}{ }^{\mathrm{i}} \mathrm{Pr}_{2}\right), 7.10\left(2 \mathrm{H}, \mathrm{d},{ }^{3} J=7.7 \mathrm{~Hz}, m_{\mathrm{b}}-\mathrm{C}_{6} \underline{\mathrm{H}}_{3}{ }^{\mathrm{i}} \operatorname{Pr}_{2}\right), 6.93\left(4 \mathrm{H}, \mathrm{m}, \mathrm{C}_{6} \underline{\mathrm{H}}_{4} \mathrm{Me}\right), 6.59(1 \mathrm{H}, \mathrm{s}$, $\mathrm{NC}(\underline{\mathrm{H}})=\mathrm{CTol}), 5.98(2 \mathrm{H}, \mathrm{s}, \mathrm{BNCH}), 4.28\left(1 \mathrm{H}, \mathrm{m}, \mathrm{MeNCH}_{2} \mathrm{CH}_{2} \mathrm{NC}_{6} \mathrm{~F}_{4}\right), 3.51\left(2 \mathrm{H}\right.$, sept., ${ }^{3} J=6.9$ $\left.\mathrm{Hz}, \underline{\mathrm{CH}}_{\mathrm{a}} \mathrm{MeMe}\right), 3.34\left(2 \mathrm{H}\right.$, sept., $\left.{ }^{3} \mathrm{~J}=6.9 \mathrm{~Hz}, \mathrm{C}_{\mathrm{b}} \mathrm{MeMe}\right), 3.14(2 \mathrm{H}$, overlapping $2 \times \mathrm{m}$, $\mathrm{MeNC}_{2} \mathrm{CH}_{2} \mathrm{NC}_{6} \mathrm{~F}_{4}$ and $\left.\mathrm{MeNC}_{2} \mathrm{CH}_{2} \mathrm{NC}_{6} \mathrm{~F}_{5}\right), 2.82\left(3 \mathrm{H}\right.$, overlapping $2 \times \mathrm{m}, \mathrm{MeNCH}_{2} \underline{\mathrm{C}}_{2} \mathrm{NC}_{6} \mathrm{~F}_{4}$ 
and $\left.\mathrm{MeNCH}_{2} \underline{\mathrm{C}}_{2} \mathrm{NC}_{6} \mathrm{~F}_{5}\right), 2.37$ (3 $\left.\mathrm{H}, \mathrm{s}, \mathrm{NMe}\right), 2.17$ (3 $\left.\mathrm{H}, \mathrm{s}, \mathrm{C}_{6} \mathrm{H}_{4} \underline{\mathrm{Me}}\right), 2.03$ (1 $\mathrm{H}, \mathrm{m}$, $\left.\mathrm{MeNC}_{2} \mathrm{CH}_{2} \mathrm{NC}_{6} \mathrm{~F}_{5}\right), 1.76\left(1 \mathrm{H}, \mathrm{m}, \mathrm{MeNC}_{2} \mathrm{CH}_{2} \mathrm{NC}_{6} \mathrm{~F}_{4}\right), 1.40\left(12 \mathrm{H}, \mathrm{d},{ }^{3} \mathrm{~J}=6.9 \mathrm{~Hz}\right.$, overlapped

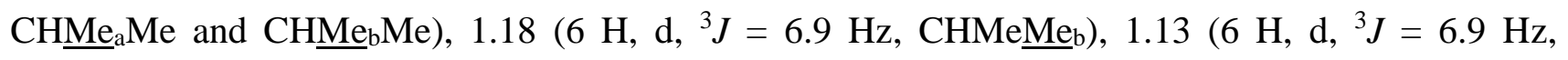
CHMeMe $\left.\underline{a}_{a}\right) .{ }^{13} \mathrm{C}\left\{{ }^{1} \mathrm{H}\right\}$ NMR $\left(\mathrm{C}_{6} \mathrm{D}_{6}, 100.6 \mathrm{MHz}\right): \delta 146.9\left(o_{\mathrm{a}}-\underline{\mathrm{C}}_{6} \mathrm{H}_{3}{ }^{\mathrm{i}} \operatorname{Pr}_{2}\right), 145.9\left(o_{\mathrm{b}}-\underline{\mathrm{C}}_{6} \mathrm{H}_{3}{ }^{\mathrm{i}} \operatorname{Pr}_{2}\right), 138.7$ $\left(i_{\mathrm{a}}-\underline{\mathrm{C}}_{6} \mathrm{H}_{3}{ }^{\mathrm{i}} \mathrm{Pr}_{2}\right), 138.0\left(i-\underline{\mathrm{C}}_{6} \mathrm{H}_{4} \mathrm{Me}\right), 137.9\left(i_{\mathrm{b}}-\underline{\mathrm{C}}_{6} \mathrm{H}_{3}{ }^{\mathrm{i}} \mathrm{Pr}_{2}\right), 130.2(\mathrm{NC}(\mathrm{H})=\mathrm{CTol}), 129.4\left(o-\underline{\mathrm{C}}_{6} \mathrm{H}_{4} \mathrm{Me}\right), 129.3$ (p- $\left.\underline{\mathrm{C}}_{6} \mathrm{H}_{4} \mathrm{Me}\right), 126.4\left(m-\underline{\mathrm{C}}_{6} \mathrm{H}_{4} \mathrm{Me}\right), 124.0\left(m_{\mathrm{a}}-\underline{\mathrm{C}}_{6} \mathrm{H}_{3}{ }_{3}{ }^{\mathrm{P}}{ }_{2}\right), 123.0\left(m_{\mathrm{b}}-\underline{\mathrm{C}}_{6} \mathrm{H}_{3}{ }^{\mathrm{i}} \operatorname{Pr}_{2}\right), 119.4(\mathrm{NCH}), 114.6$ $(\mathrm{NC}(\mathrm{H})=\underline{\mathrm{CTO}} \mathrm{T}), 55.1\left(\mathrm{MeNCH}_{2} \underline{\mathrm{CH}}_{2} \mathrm{NC}_{6} \mathrm{~F}_{5}\right), 55.5\left(\mathrm{MeNCH}_{2} \mathrm{CH}_{2} \mathrm{NC}_{6} \mathrm{~F}_{5}\right), 54.0\left(\mathrm{MeNCH}_{2} \underline{\mathrm{CH}}_{2} \mathrm{NC}_{6} \mathrm{~F}_{4}\right)$, $51.7\left(\mathrm{MeNCH}_{2} \mathrm{CH}_{2} \mathrm{NC}_{6} \mathrm{~F}_{4}\right), 43.2$ (NMe), 28.6 ( $\left.\underline{\mathrm{CH}}_{\mathrm{a}} \mathrm{MeMe}\right), 28.3$ ( $\left.\underline{\mathrm{CH}}_{\mathrm{b}} \mathrm{MeMe}\right), 26.7$ ( $\left.\mathrm{CHMeMe}_{\mathrm{a}}\right)$,

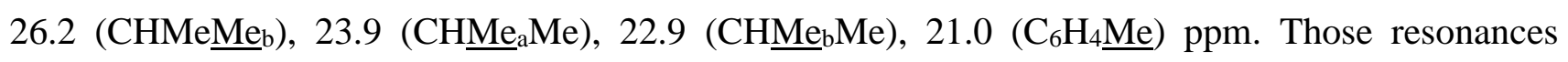
belonging to the $\mathrm{NAr}^{\mathrm{F}}$ and $\mathrm{C}_{6} \mathrm{~F}_{4}$ rings could not be satisfactorily assigned. ${ }^{11} \mathrm{~B}\left\{{ }^{1} \mathrm{H}\right\} \mathrm{NMR}\left(\mathrm{C}_{6} \mathrm{D}_{6}\right.$, 128.4 MHz): $\delta 22.9$ ppm. ${ }^{19} \mathrm{~F}\left\{{ }^{1} \mathrm{H}\right\}$ NMR $\left(\mathrm{C}_{6} \mathrm{D}_{6}, 376.5 \mathrm{MHz}\right):-102.5$ (s, TiF), -128.9 (1 F, m, $\delta$ $\left.\mathrm{C}_{6} \mathrm{~F}_{4}\right),-146.4\left(1 \mathrm{~F}, \mathrm{dd},{ }^{3} J=20.2 \mathrm{~Hz},{ }^{4} J=7.8 \mathrm{~Hz}, \beta-\mathrm{C}_{6} \mathrm{~F}_{4}\right),-147.0\left(2 \mathrm{~F}, \mathrm{~d},{ }^{3} J=23.4 \mathrm{~Hz}, o-\mathrm{C}_{6} \mathrm{~F}_{5}\right),-$ 158.1 ( $1 \mathrm{~F}$, app. t, app. $\left.{ }^{3} J=21.0 \mathrm{~Hz}, \alpha-\mathrm{C}_{6} \mathrm{~F}_{4}\right),-163.0\left(1 \mathrm{~F}, \mathrm{~m}, p-\mathrm{C}_{6} \mathrm{~F}_{5}\right),-163.3\left(2 \mathrm{~F}, \mathrm{~m}, m-\mathrm{C}_{6} \mathrm{~F}_{5}\right),-$ 164.8 ( $1 \mathrm{~F}$, app. t, $\left.J=23.1 \mathrm{~Hz}, \gamma-\mathrm{C}_{6} \mathrm{~F}_{4}\right)$ ppm. IR ( $\mathrm{NaCl}$ plates, Nujol mull, $\left.\mathrm{cm}^{-1}\right): 1634(\mathrm{w}), 1607(\mathrm{w})$, 1586 (w), 1557 (w), 1502 (s), 1363 (s), 1335 (s), 1289 (m), 1203 (w), 1115 (m), 1060 (s), 997 (s), 989 (s), $951(\mathrm{w}), 899(\mathrm{~m}), 858(\mathrm{~m}), 821(\mathrm{w}), 786(\mathrm{w}), 758(\mathrm{~m}), 690(\mathrm{w}), 656(\mathrm{~m}), 623(\mathrm{~m}), 601(\mathrm{~m})$. EI-MS: $m / z=1012[M]^{+}(100 \%)$. Anal. found (calcd. for $\mathrm{C}_{52} \mathrm{H}_{55} \mathrm{BF}_{10} \mathrm{~N}_{6} \mathrm{Ti}$ ): C, 61.47 (61.67); H, 5.61 (5.47); N, $8.11(8.30) \%$.

NMR tube scale synthesis of $\operatorname{Ti}\left\{\mathrm{MeN}\left(\mathrm{CH}_{2} \mathrm{CH}_{2} \mathrm{NAr}^{\mathrm{F}}\right)\left\{\mathrm{CH}_{2} \mathrm{CH}_{2} \mathrm{NC}_{6} \mathrm{~F}_{4} \mathrm{C}(\mathrm{Tol})-\right.\right.$ CHNB(NAr'CH)2 $\}$ \}(F) (17). To an NMR tube fitted with a J. Youngs valve was added a solution of $\mathrm{Ti}\left(\mathrm{N}_{2}{ }^{\mathrm{ArF}} \mathrm{N}^{\mathrm{Me}}\right)\left\{\mathrm{N}\left\{\mathrm{B}\left(\mathrm{NAr}{ }^{\prime} \mathrm{CH}\right)_{2}\right\} \mathrm{C}(\mathrm{H}) \mathrm{C}(\mathrm{Tol})\right\}(\mathbf{1 4}, 0.0150 \mathrm{~g}, 0.0148 \mathrm{mmol})$ in $\mathrm{C}_{6} \mathrm{D}_{6}(0.6 \mathrm{~mL})$, and the tube heated to $60{ }^{\circ} \mathrm{C}$ for $16 \mathrm{~h}$, after which time the ${ }^{1} \mathrm{H}$ NMR spectrum indicated quantitative conversion to 17.

Kinetic measurements for the conversion of $\operatorname{Ti}\left(\mathrm{N}_{2}{ }^{\mathrm{ArF}} \mathrm{N}^{\mathrm{Me}}\right)\left\{\mathrm{N}\left\{\mathrm{B}\left(\mathrm{NAr} \mathbf{r}^{\prime} \mathrm{CH}\right)_{2}\right\} \mathrm{C}(\mathrm{H}) \mathrm{C}(\mathrm{Tol})\right\}$ (14) to $\mathrm{Ti}\left\{\mathrm{MeN}\left(\mathrm{CH}_{2} \mathrm{CH}_{2} \mathrm{NAr}^{\mathrm{F}}\right)\left\{\mathrm{CH}_{2} \mathrm{CH}_{2} \mathrm{NC}_{6} \mathrm{~F}_{4} \mathrm{C}(\mathrm{Tol}) \mathrm{CHNB}\left(\mathrm{NAr}^{\prime} \mathrm{CH}\right)_{2}\right\}\right\}(\mathbf{F}) \quad(17)$ The general procedure is as follows. In a dry-box, a $\mathrm{C}_{6} \mathrm{D}_{6}$ solution $(0.6 \mathrm{~mL})$ of $\mathrm{Ti}\left(\mathrm{N}_{2}{ }^{\mathrm{ArF}} \mathrm{N}^{\mathrm{Me}}\right)\left\{\mathrm{N}\left\{\mathrm{B}\left(\mathrm{NAr}{ }^{\prime} \mathrm{CH}\right)_{2}\right\} \mathrm{C}(\mathrm{H}) \mathrm{C}(\mathrm{Tol})\right\} \quad(\mathbf{1 4}, 0.0156 \mathrm{~g}, 0.0154 \mathrm{mmol})$ was transferred to an NMR tube fitted with a J. Youngs valve. A blank $\mathrm{C}_{6} \mathrm{D}_{6}$ sample was added to the NMR probe at RT, locked and shimmed, and the probe heated to the relevant temperature (327, 330, 333, 336 or $339 \mathrm{~K})$. After thermal equilibration, the experimental sample replaced the blank sample, the shimming was checked and an array was set up to record a ${ }^{1} \mathrm{H}$ NMR spectrum (4 scans) every $120 \mathrm{~s}$. The formation 
of 17 was calculated by measuring the integral of NMe. First order rate constants were obtained from linear plots of $-\ln \left(1-\left([\mathbf{1 7}] /[\mathbf{1 7}]_{\text {final }}\right)\right)$ vs time.

Kinetic measurements for the conversion of $\operatorname{Ti}\left(\mathrm{N}_{2}{ }^{\mathrm{ArF}} \mathbf{N}^{\mathrm{Me}}\right)\left\{\mathrm{N}\left\{\mathrm{B}\left(\mathrm{NAr} \mathbf{r}^{\prime} \mathrm{CH}\right)_{2}\right\} \mathrm{C}(\mathrm{H}) \mathrm{C}\left(\mathrm{Ar} \mathbf{r}^{\mathrm{F}}\right)\right\}$ (16) to $\operatorname{Ti}\left\{\mathrm{MeN}\left(\mathrm{CH}_{2} \mathrm{CH}_{2} \mathrm{NAr}^{\mathrm{F}}\right)\left\{\mathrm{CH}_{2} \mathrm{CH}_{2} \mathrm{NC}_{6} \mathrm{~F}_{4} \mathrm{C}\left(\mathrm{Ar}^{\mathrm{F}}\right)=\mathrm{CHNB}\left(\mathrm{NAr}^{\prime} \mathrm{CH}\right)_{2}\right\}\right\}(\mathbf{F}) \quad$ (15). The general procedure is as follows. In a dry-box, $\mathrm{Ti}_{(}\left(\mathrm{N}_{2}{ }^{\mathrm{ArF}} \mathrm{N}^{\mathrm{Me}}\right)\left\{\mathrm{NB}\left(\mathrm{NAr}{ }^{\prime} \mathrm{CH}\right)_{2}\right\}($ py) $(\mathbf{1 0}, 0.0150 \mathrm{~g}, 0.0154$ mmol) and $\mathrm{B}\left(\mathrm{Ar}^{\mathrm{F}}\right)_{3}(0.0079 \mathrm{~g}, 0.0154 \mathrm{mmol})$ were mixed in $\mathrm{C}_{6} \mathrm{D}_{6}(0.6 \mathrm{~mL})$, and the mixture transferred to an NMR tube fitted with a J. Youngs valve. Next, $\operatorname{Ar}^{\mathrm{F} C C H}(2.0 \mu \mathrm{L}, 0.0154 \mathrm{mmol})$ was added via microsyringe, giving immediate conversion to 16. A blank $\mathrm{C}_{6} \mathrm{D}_{6}$ sample was added to the NMR probe at RT, locked and shimmed, and the probe heated to the relevant temperature $(327,330$, 333, 336 or $339 \mathrm{~K})$. After thermal equilibration, the experimental sample replaced the blank sample, the shimming was checked and an array was set up to record a ${ }^{1} \mathrm{H}$ NMR spectrum (4 scans) every 120 s. The formation of 15 was calculated by measuring the integral of NMe. First order rate constants were obtained from linear plots of $-\ln \left(1-\left([\mathbf{1 5}] /[\mathbf{1 5}]_{\text {final }}\right)\right) v s$ time.

$\mathbf{T i}\left(\mathbf{N}_{2}{ }^{i P r} \mathbf{N}^{M e}\right)\left\{\mathbf{N}\left\{\mathbf{B}\left(\mathbf{N A r} \mathbf{C}^{\prime} \mathbf{C H}\right)_{2}\right\} \mathbf{C}(\mathbf{H}) \mathbf{C}(\mathbf{T o l})\right\}$ (18). To a solution of $\operatorname{Ti}\left(\mathrm{N}_{2}{ }^{i \mathrm{Pr}} \mathrm{N}^{\mathrm{Me}}\right)\left\{\mathrm{NB}\left(\mathrm{NAr}{ }^{\prime} \mathrm{CH}\right)_{2}\right\}($ py) (11, $0.500 \mathrm{~g}, 0.687 \mathrm{mmol})$ in toluene $(15 \mathrm{~mL})$ was added TolCCH $(87 \mu \mathrm{L}, 0.686 \mathrm{mmol})$ at RT, giving an immediate color change to deep brown. The mixture was stirred for $1 \mathrm{~h}$, then the volatiles removed under reduced pressure. The product was dissolved in hexane $(5 \mathrm{~mL})$, concentrated to $2 \mathrm{~mL}$ then cooled to $-30{ }^{\circ} \mathrm{C}$, resulting in large, deep brown crystals of 18 . Yield: $0.228 \mathrm{~g}$ (43\%). Diffraction-quality crystals were grown from a concentrated hexane solution at $5{ }^{\circ} \mathrm{C}$. Major isomer (cis-18): ${ }^{1} \mathrm{H}$ NMR $\left(\mathrm{C}_{6} \mathrm{D}_{6}, 400.1 \mathrm{MHz}\right): \delta 10.32(1 \mathrm{H}, \mathrm{s}, \mathrm{TiC}=\mathrm{CH}), 7.32-7.20(6 \mathrm{H}$, overlapping $2 \times$ $\mathrm{m}$ with minor isomer, $m$ - and $\left.p-\mathrm{C}_{6} \underline{\mathrm{H}}_{3}{ }^{\mathrm{i}} \mathrm{Pr}_{2}\right), 7.04\left(2 \mathrm{H}, \mathrm{d},{ }^{3} \mathrm{~J}=8.0 \mathrm{~Hz}, o-\mathrm{C}_{6} \underline{\mathrm{H}}_{4} \mathrm{Me}\right), 6.93\left(2 \mathrm{H}, \mathrm{d},{ }^{3} J=\right.$ $\left.8.0 \mathrm{~Hz}, m-\mathrm{C}_{6} \underline{\mathrm{H}}_{4} \mathrm{Me}\right), 5.99(2 \mathrm{H}, \mathrm{s}, \mathrm{BNCH}), 4.26\left(2 \mathrm{H}\right.$, sept., $\left.{ }^{3} J=6.7 \mathrm{~Hz}, \mathrm{NC} \underline{\mathrm{HMeMe}}\right), 3.56(4 \mathrm{H}$, sept. overlapping with minor isomer, $\left.{ }^{3} J=6.9 \mathrm{~Hz}, \mathrm{C}_{6} \mathrm{H}_{3}\left(\mathrm{C}_{\underline{H} M e}\right)_{2}\right), 3.40\left(2 \mathrm{H}, \mathrm{m}, \mathrm{C}_{2} \mathrm{~N}^{\mathrm{i}} \mathrm{Pr}\right), 2.99$ (4 $\mathrm{H}$, overlapping $2 \times \mathrm{m}$ with minor isomer, $\mathrm{C}_{2} \mathrm{~N}^{\mathrm{i}} \mathrm{Pr}$ and $\left.\mathrm{C}_{2} \mathrm{NMe}\right), 2.21\left(2 \mathrm{H}, \mathrm{m}, \mathrm{C}_{2} \mathrm{NMe}\right), 2.14$ (3 $\left.\mathrm{H}, \mathrm{s}, \mathrm{C}_{6} \mathrm{H}_{4} \underline{\mathrm{Me}}\right), 1.29\left(12 \mathrm{H}\right.$, d overlapping with minor isomer, ${ }^{3} \mathrm{~J}=6.9 \mathrm{~Hz}, \mathrm{C}_{6} \mathrm{H}_{3}(\mathrm{CHMeMe}) 2$ ), 1.08 (3 $\mathrm{H}, \mathrm{s}, \mathrm{NMe}), 1.00\left(6 \mathrm{H}, \mathrm{d},{ }^{3} J=6.7 \mathrm{~Hz}, \mathrm{NCHMeMe}\right), 0.91\left(6 \mathrm{H}, \mathrm{d},{ }^{3} J=6.7 \mathrm{~Hz}, \mathrm{NCHMeMe}\right) \mathrm{ppm}$. ${ }^{13} \mathrm{C}\left\{{ }^{1} \mathrm{H}\right\}$ NMR $\left(\mathrm{C}_{6} \mathrm{D}_{6}, 100.6 \mathrm{MHz}\right): \delta 209.5(\mathrm{TiC}=\mathrm{CH}), 148.9(\mathrm{TiC}=\underline{\mathrm{CH}}), 147.3\left(o-\underline{\mathrm{C}}_{6} \mathrm{H}_{3}{ }^{\mathrm{i}} \mathrm{Pr}_{2}\right), 142.0$ (i- $\left.\underline{\mathrm{C}}_{6} \mathrm{H}_{3}{ }^{\mathrm{i}} \mathrm{Pr}_{2}\right), 139.7\left(i-\underline{\mathrm{C}}_{6} \mathrm{H}_{4} \mathrm{Me}\right), 133.5$ (p- $\left.\underline{\mathrm{C}}_{6} \mathrm{H}_{4} \mathrm{Me}\right), 129.2\left(o-\underline{\mathrm{C}}_{6} \mathrm{H}_{4} \mathrm{Me}\right), 127.5\left(p-\underline{\mathrm{C}}_{6} \mathrm{H}_{3}{ }^{i} \operatorname{Pr}_{2}\right), 127.0$ (m$\left.\underline{\mathrm{C}}_{6} \mathrm{H}_{4} \mathrm{Me}\right), 124.1$ ( $\left.m-\underline{\mathrm{C}}_{6} \mathrm{H}_{3}{ }^{\mathrm{i}} \mathrm{Pr}_{2}\right), 118.6(\mathrm{BNCH}), 57.2$ (NCHMeMe), $56.9\left(\underline{\mathrm{CH}}_{2} \mathrm{NMe}\right), 47.2\left(\underline{\mathrm{CH}}_{2} \mathrm{~N}^{\mathrm{i}} \mathrm{Pr}\right)$, 41.5 (NMe), $\left.28.9\left(\mathrm{C}_{6} \mathrm{H}_{3}(\underline{\mathrm{C}} \mathrm{HMeMe})_{2}\right), 24.6\left(\mathrm{C}_{6} \mathrm{H}_{3}(\mathrm{CHMeMe})_{2}\right), 23.9\left(\mathrm{C}_{6} \mathrm{H}_{3}(\mathrm{CHMeMe})\right)_{2}\right), 23.6$ (NCHMeMe), 22.2 (NCHMeMe), $21.2\left(\mathrm{C}_{6} \mathrm{H}_{4} \underline{\mathrm{Me}}\right)$ ppm. Minor isomer (trans-18): ${ }^{1} \mathrm{H}$ NMR $\left(\mathrm{C}_{6} \mathrm{D}_{6}\right.$, $400.1 \mathrm{MHz}): \delta 9.35(1 \mathrm{H}, \mathrm{s}, \mathrm{TiC}=\mathrm{CH}), 7.32-7.20(6 \mathrm{H}$, overlapping $2 \times \mathrm{m}$ with major isomer, $m$ - 
and $\left.p-\mathrm{C}_{6} \underline{\mathrm{H}}_{3}{ }^{\mathrm{i}} \mathrm{Pr}_{2}\right), 6.94\left(2 \mathrm{H}\right.$, overlapping with major isomer, $\left.o-\mathrm{C}_{6} \underline{\mathrm{H}}_{4} \mathrm{Me}\right), 6.71\left(2 \mathrm{H}, \mathrm{d},{ }^{3} \mathrm{~J}=8.0 \mathrm{~Hz}, m\right.$ $\left.\mathrm{C}_{6} \underline{\mathrm{H}}_{4} \mathrm{Me}\right), 6.02(2 \mathrm{H}, \mathrm{s}, \mathrm{BNCH}), 4.18\left(2 \mathrm{H}\right.$, sept., $\left.{ }^{3} J=6.7 \mathrm{~Hz}, \mathrm{NC} \underline{\mathrm{HMeMe}}\right), 3.56$ (2 H, sept. overlapping with major isomer, $\left.{ }^{3} J=6.9 \mathrm{~Hz}, \mathrm{C}_{6} \mathrm{H}_{3}\left(\mathrm{C}_{\underline{H} M e}\right)_{2}\right), 3.25\left(2 \mathrm{H}, \mathrm{m}, \mathrm{C}_{2} \mathrm{~N}^{\mathrm{i}} \mathrm{Pr}\right), 2.99(4 \mathrm{H}$, overlapping $2 \times \mathrm{m}$ with major isomer, $\underline{\mathrm{C}}_{2} \mathrm{~N}^{\mathrm{i}} \mathrm{Pr}$ and $\left.\underline{\mathrm{C}}_{2} \mathrm{NMe}\right), 2.42\left(2 \mathrm{H}, \mathrm{m}, \underline{\mathrm{C}}_{2} \mathrm{NMe}\right), 2.09$ (3 H, s, $\left.\mathrm{C}_{6} \mathrm{H}_{4} \underline{\mathrm{Me}}\right), 1.35\left(12 \mathrm{H}, \mathrm{d},{ }^{3} J=6.9 \mathrm{~Hz}, \mathrm{C}_{6} \mathrm{H}_{3}(\mathrm{CHMeMe})_{2}, 1.30(3 \mathrm{H}, \mathrm{s}, \mathrm{NMe}), 1.29(12 \mathrm{H}, \mathrm{d}\right.$ overlapping with major isomer, $\left.{ }^{3} \boldsymbol{J}=6.9 \mathrm{~Hz}, \mathrm{C}_{6} \mathrm{H}_{3}(\mathrm{CHMeMe})_{2}\right), 0.95\left(6 \mathrm{H}, \mathrm{d},{ }^{3} \boldsymbol{J}=6.7 \mathrm{~Hz}\right.$, $\mathrm{NCHMeMe}), 0.76\left(6 \mathrm{H}, \mathrm{d},{ }^{3} J=6.7 \mathrm{~Hz}, \mathrm{NCHMeMe}\right) \mathrm{ppm} .{ }^{13} \mathrm{C}\left\{{ }^{1} \mathrm{H}\right\} \mathrm{NMR}\left(\mathrm{C}_{6} \mathrm{D}_{6}, 100.6 \mathrm{MHz}\right): \delta$ $196.7(\mathrm{Ti} \underline{\mathrm{C}}=\mathrm{CH}), 147.2\left(o-\underline{\mathrm{C}}_{6} \mathrm{H}_{3}{ }^{\mathrm{i}} \mathrm{Pr}_{2}\right), 144.8\left(i-\underline{\mathrm{C}}_{6} \mathrm{H}_{4} \mathrm{Me}\right), 143.5(\mathrm{TiC}=\underline{\mathrm{CH}}), 141.3\left(i-\underline{\mathrm{C}}_{6} \mathrm{H}_{3}{ }^{\mathrm{i}} \operatorname{Pr}_{2}\right), 132.6$ (p- $\left.\underline{\mathrm{C}}_{6} \mathrm{H}_{4} \mathrm{Me}\right), 129.0\left(o-\underline{\mathrm{C}}_{6} \mathrm{H}_{4} \mathrm{Me}\right), 127.5\left(p-\underline{\mathrm{C}}_{6} \mathrm{H}_{3}{ }^{\mathrm{i}} \operatorname{Pr}_{2}\right), 125.7\left(m-\underline{\mathrm{C}}_{6} \mathrm{H}_{4} \mathrm{Me}\right), 123.9\left(m-\underline{\mathrm{C}}_{6} \mathrm{H}_{3}{ }^{\mathrm{i}} \operatorname{Pr}_{2}\right), 119.6$

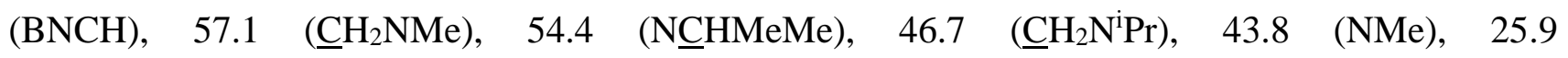
$\left(\mathrm{C}_{6} \mathrm{H}_{3}(\underline{\mathrm{CHMeMe}})_{2}\right), 23.7$ ( $\left.\left.\mathrm{C}_{6} \mathrm{H}_{3}(\mathrm{CHMeMe})\right)_{2}\right), 23.7$ (NCHMeMe), 21.6 (NCHMe $\left.\underline{\mathrm{Ne}}\right), 21.0\left(\mathrm{C}_{6} \mathrm{H}_{4} \underline{\mathrm{Me}}\right)$ ppm. Common data: ${ }^{11} \mathrm{~B}\left\{{ }^{1} \mathrm{H}\right\}$ NMR $\left(\mathrm{C}_{6} \mathrm{D}_{6}, 128.4 \mathrm{MHz}\right): 22.1 \mathrm{ppm}$. IR (NaCl plates, Nujol mull, $\mathrm{cm}^{-}$ 1): 2078 (w), 1930 (w), 1867 (w), 1656 (s), 1585 (m), 1568 (w), 1504 (m), 1487 (m), 1361 (m), 1276 (m), 1227 (w), 1116 (m), 1104 (m), 1042 (w), 994 (w), 944 (m), 898 (w), 762 (m), 648 (m). EI-MS: $m / z=519\left[\mathrm{TolC}(\mathrm{H}) \mathrm{C}(\mathrm{H}) \mathrm{N}(\mathrm{H}) \mathrm{B}\left(\mathrm{NAr}{ }^{\prime} \mathrm{CH}\right)_{2}\right]^{+}(95 \%)$. Anal. found (calcd. for $\left.\mathrm{C}_{46} \mathrm{H}_{69} \mathrm{BN} 6 \mathrm{Ti}\right): \mathrm{C}, 72.12$ (72.24); H, 9.09 (9.09); N, 10.88 (10.99)\%.

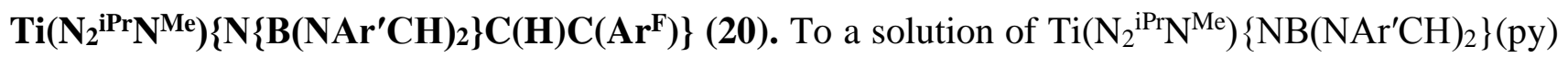
$(11,0.300 \mathrm{~g}, 0.412 \mathrm{mmol})$ in toluene $(10 \mathrm{~mL})$ was added $\operatorname{Ar}^{\mathrm{F}} \mathrm{CCH}(55 \mu \mathrm{L}, 0.412 \mathrm{mmol})$ at RT. The mixture was heated to $60{ }^{\circ} \mathrm{C}$, stirred for $16 \mathrm{~h}$ and then the volatiles removed under reduced pressure to yield 20 as a brown, waxy solid. Yield: $0.253 \mathrm{~g}(73 \%)$. Major isomer (trans-20): ${ }^{1} \mathrm{H}$ NMR $\left(\mathrm{C}_{6} \mathrm{D}_{6}\right.$, $500.3 \mathrm{MHz}$ ): $\delta 9.29(1 \mathrm{H}, \mathrm{s}, \mathrm{TiC}=\mathrm{CH}$ ), $7.28-7.17$ (multiple m overlapping with minor isomer, $m$ and $\left.p-\mathrm{C}_{6} \underline{\mathrm{H}}_{3}{ }^{\mathrm{i}} \mathrm{Pr}_{2}\right), 5.97(2 \mathrm{H}, \mathrm{s}, \mathrm{BNCH}), 4.27\left(2 \mathrm{H}\right.$, sept. overlapping with minor isomer, ${ }^{3} \mathrm{~J}=6.5 \mathrm{~Hz}$, $\mathrm{NC} \underline{\mathrm{HMeMe}}$ ), 3.50 (4 H, sept. overlapping with minor isomer, $\left.{ }^{3} J=6.9 \mathrm{~Hz}, \mathrm{C}_{6} \mathrm{H}_{3}(\mathrm{C} \underline{\mathrm{HMeMe}})_{2}\right), 3.22$ ( $2 \mathrm{H}, \mathrm{m}, \underline{\mathrm{C}}_{2} \mathrm{~N}^{\mathrm{i}} \mathrm{Pr}$ ), 2.89 (4 H, overlapping $2 \times \mathrm{m}, \underline{\mathrm{C}}_{2} \mathrm{NMe}$ and $\underline{\mathrm{C}}_{2} \mathrm{~N}^{\mathrm{i}} \mathrm{Pr}$ ), $2.40\left(2 \mathrm{H}, \mathrm{m}, \mathrm{C}_{2} \mathrm{NMe}\right)$, 1.89 (3 H, s, NMe), $1.37\left(12 \mathrm{H}, \mathrm{d},{ }^{3} J=6.9 \mathrm{~Hz}, \mathrm{C}_{6} \mathrm{H}_{3}(\mathrm{CHMeMe})_{2}\right), 1.29-1.25$ (12 H, d overlapping with minor isomer, $\left.\mathrm{C}_{6} \mathrm{H}_{3}(\mathrm{CHMeMe})_{2}\right), 0.97\left(6 \mathrm{H}, \mathrm{d},{ }^{3} J=6.5 \mathrm{~Hz}, \mathrm{NCHMeMe}\right), 0.71\left(6 \mathrm{H}, \mathrm{d},{ }^{3} J=6.5\right.$ $\mathrm{Hz}, \mathrm{NCHMe} \underline{\underline{\mathrm{Me}}})$ ppm. ${ }^{13} \mathrm{C}\left\{{ }^{1} \mathrm{H}\right\}$ NMR $\left(\mathrm{C}_{6} \mathrm{D}_{6}, 125.7 \mathrm{MHz}\right): \delta 168.7(\mathrm{TiC}=\mathrm{CH}), 147.6(\mathrm{TiC}=\underline{\mathrm{C}} \mathrm{H})$, $147.0\left(o-\underline{\mathrm{C}}_{6} \mathrm{H}_{3}{ }^{\mathrm{i}} \mathrm{Pr}_{2}\right), 142.7$ (m, o- $\left.\mathrm{C}_{6} \mathrm{~F}_{5}\right), 140.8\left(i-\underline{\mathrm{C}}_{6} \mathrm{H}_{3}{ }^{\mathrm{i}} \mathrm{Pr}_{2}\right), 140.4$ (m, m- $\left.\mathrm{C}_{6} \mathrm{~F}_{5}\right), 136.9$ (m, $\left.p-\mathrm{C}_{6} \mathrm{~F}_{5}\right)$, $127.8\left(p-\underline{\mathrm{C}}_{6} \mathrm{H}_{3}{ }^{\mathrm{i}} \mathrm{Pr}_{2}\right), 124.0\left(m-\underline{\mathrm{C}}_{6} \mathrm{H}_{3}{ }^{\mathrm{i}} \mathrm{Pr}_{2}\right), 122.2\left(\mathrm{td},{ }^{2} J_{\mathrm{C}-\mathrm{F}}=17.9 \mathrm{~Hz},{ }^{3} J_{\mathrm{C}-\mathrm{F}}=3.5 \mathrm{~Hz}, i-\mathrm{C}_{6} \mathrm{~F}_{5}\right), 119.9$

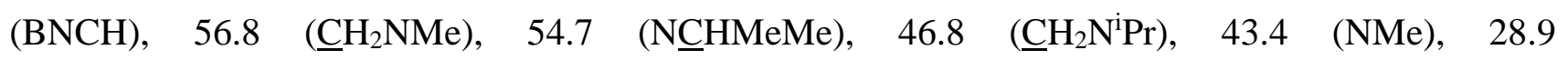
$\left.\left(\mathrm{C}_{6} \mathrm{H}_{3}(\underline{\mathrm{CHMeMe}})_{2}\right), 25.9\left(\mathrm{C}_{6} \mathrm{H}_{3}(\mathrm{CHMeMe})\right)_{2}\right), 23.7\left(\mathrm{C}_{6} \mathrm{H}_{3}(\mathrm{CHMeMe})_{2}\right), 23.0$ (NCHMeMe), 21.5 (NCHMeMe $)$ ppm. ${ }^{19} \mathrm{~F}\left\{{ }^{1} \mathrm{H}\right\}$ NMR $\left(\mathrm{C}_{6} \mathrm{D}_{6}, 376.5 \mathrm{MHz}\right):-144.1\left(2 \mathrm{~F}, \mathrm{~m}, o-\mathrm{C}_{6} \mathrm{~F}_{5}\right),-164.5\left(1 \mathrm{~F}, \mathrm{t},{ }^{3} J=\right.$ $\left.21.5 \mathrm{~Hz}, p-\mathrm{C}_{6} \mathrm{~F}_{5}\right),-165.6\left(2 \mathrm{~F}, \mathrm{~m}, m-\mathrm{C}_{6} \mathrm{~F}_{5}\right)$. Minor isomer (cis-20): ${ }^{1} \mathrm{H} \mathrm{NMR}\left(\mathrm{C}_{6} \mathrm{D}_{6}, 500.3 \mathrm{MHz}\right): \delta$ 
$10.32(1 \mathrm{H}, \mathrm{s}, \mathrm{TiC}=\mathrm{CH}), 7.28-7.17$ (multiple m overlapping with major isomer, $m$ - and $p-\mathrm{C}_{6} \underline{\mathrm{H}}_{3}{ }^{\mathrm{i}} \mathrm{Pr}_{2}$ ), $6.17(2 \mathrm{H}, \mathrm{s}, \mathrm{BNCH}), 4.27$ (2 H, sept. overlapping with major isomer, $\left.{ }^{3} J=6.5 \mathrm{~Hz}, \mathrm{NC} \underline{\mathrm{HMeMe}}\right), 3.50$ (4 H, sept. overlapping with major isomer, $\left.{ }^{3} \mathrm{~J}=6.9 \mathrm{~Hz}, \mathrm{C}_{6} \mathrm{H}_{3}(\mathrm{C} \underline{\mathrm{HMeMe}})_{2}\right), 3.31\left(2 \mathrm{H}, \mathrm{m}, \mathrm{C}_{2} \mathrm{~N}^{\mathrm{i}} \mathrm{Pr}\right.$ ), $2.95\left(2 \mathrm{H}, \mathrm{m}, \mathrm{C}_{2} \mathrm{~N}^{\mathrm{i}} \mathrm{Pr}\right), 2.75\left(2 \mathrm{H}, \mathrm{m}, \mathrm{CH}_{2} \mathrm{NMe}\right), 2.17\left(2 \mathrm{H}, \mathrm{m}, \mathrm{CH}_{2} \mathrm{NMe}\right), 1.29-1.25(24 \mathrm{H}, 2 \times \mathrm{d}$ overlapping with major isomer, $\left.\mathrm{C}_{6} \mathrm{H}_{3}(\mathrm{CHMeMe}) 2\right), 1.10(3 \mathrm{H}, \mathrm{s}, \mathrm{NMe}), 0.94\left(6 \mathrm{H}, \mathrm{d},{ }^{3} \mathrm{~J}=6.5 \mathrm{~Hz}\right.$, $\mathrm{NCHMeMe}), 0.90\left(6 \mathrm{H}, \mathrm{d},{ }^{3} \mathrm{~J}=6.5 \mathrm{~Hz}, \mathrm{NCHMeMe}\right) \mathrm{ppm} .{ }^{13} \mathrm{C}\left\{{ }^{1} \mathrm{H}\right\} \mathrm{NMR}\left(\mathrm{C}_{6} \mathrm{D}_{6}, 125.7 \mathrm{MHz}\right): \delta$ $183.3(\mathrm{Ti} \underline{\mathrm{C}}=\mathrm{CH}), 149.7(\mathrm{TiC}=\underline{\mathrm{CH}}), 146.9\left(o-\underline{\mathrm{C}}_{6} \mathrm{H}_{3}{ }^{\mathrm{i}} \operatorname{Pr}_{2}\right), 141.2\left(i-\underline{\mathrm{C}}_{6} \mathrm{H}_{3}{ }^{\mathrm{i}} \operatorname{Pr}_{2}\right), 127.7\left(p-\underline{\mathrm{C}}_{6} \mathrm{H}_{3}{ }^{\mathrm{i}} \operatorname{Pr}_{2}\right), 124.1$

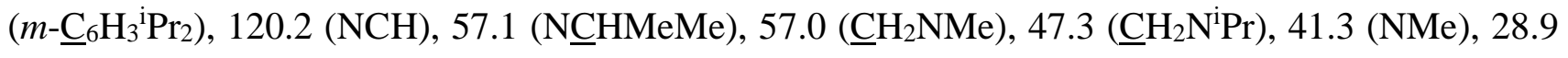
$\left(\mathrm{C}_{6} \mathrm{H}_{3}(\underline{\mathrm{CHMeMe}})_{2}\right), 24.9\left(\mathrm{C}_{6} \mathrm{H}_{3}(\mathrm{CHMeMe})_{2}\right), 23.9\left(\mathrm{C}_{6} \mathrm{H}_{3}(\mathrm{CHMeMe})_{2}\right), 22.2$ (NCHMeMe), 22.1 (NCHMeMe) ppm. Those resonances belonging to the $\operatorname{Ar}^{\mathrm{F}}$ ring could not be satisfactorily assigned. ${ }^{19} \mathrm{~F}\left\{{ }^{1} \mathrm{H}\right\}$ NMR $\left(\mathrm{C}_{6} \mathrm{D}_{6}, 376.5 \mathrm{MHz}\right): \delta-141.7\left(2 \mathrm{~F}, \mathrm{~m}, o-\mathrm{C}_{6} \mathrm{~F}_{5}\right),-164.9\left(1 \mathrm{~F}, \mathrm{t},{ }^{3} J=21.5 \mathrm{~Hz}, p-\mathrm{C}_{6} \mathrm{~F}_{5}\right),-$ 166.2 (2 F, m, m- $\left.\mathrm{C}_{6} \mathrm{~F}_{5}\right)$ ppm. Common data: ${ }^{11} \mathrm{~B}\left\{{ }^{1} \mathrm{H}\right\}$ NMR $\left(\mathrm{C}_{6} \mathrm{D}_{6}, 128.4 \mathrm{MHz}\right): 22.7 \mathrm{ppm}$. IR $(\mathrm{NaCl}$ plates, Nujol mull, cm ${ }^{-1}$ ): 3438 (w), 3065 (w), 1929 (w), 1655 (m), 1638 (m), 1585 (m), 1512 (s), 1415 (s), 1288 (w), 1228 (w), 1203 (w), 1176 (w), 1117 (m), 1007 (m), 989 (m), 976 (m), 960 (s), $940(\mathrm{w}), 898(\mathrm{w}), 888(\mathrm{w}), 865(\mathrm{w}), 823$ (s), $759(\mathrm{~s}), 674(\mathrm{w}), 649(\mathrm{w}), 620(\mathrm{w}), 606(\mathrm{~m}), 566(\mathrm{w})$. EI-MS: $m / z=595\left[\mathrm{Ar}^{\mathrm{F}} \mathrm{C}(\mathrm{H}) \mathrm{C}(\mathrm{H}) \mathrm{N}(\mathrm{H}) \mathrm{B}\left(\mathrm{NAr}{ }^{\prime} \mathrm{CH}\right)_{2}\right]^{+}(100 \%)$. Anal. found (calcd. for $\left.\mathrm{C}_{45} \mathrm{H}_{62} \mathrm{BF}_{5} \mathrm{~N}_{6} \mathrm{Ti}\right)$ : C, 64.09 (64.29); H, 7.59 (7.43); N, 9.90 (10.00)\%.

NMR tube scale synthesis of $\mathbf{T i}\left(\mathbf{N}_{2}{ }^{i P r} \mathbf{N}^{M e}\right)\left\{\mathbf{N}\left\{\mathbf{B}\left(\mathbf{N A r}^{\prime} \mathbf{C H}\right)_{2}\right\} \mathbf{C}(\mathbf{H}) \mathbf{C}\left(\mathbf{A r}^{\mathrm{F}}\right)\right\}(20)$. To an NMR tube fitted with a J. Youngs valve was added a solution of $\operatorname{Ti}\left(\mathrm{N}_{2}{ }^{\mathrm{iPr}} \mathrm{N}^{\mathrm{Me}}\right)\left\{\mathrm{NHB}\left(\mathrm{NAr}{ }^{\prime} \mathrm{CH}\right)_{2}\right\}\left(\mathrm{CCAr}^{\mathrm{F}}\right)(\mathbf{1 9}$, $0.0150 \mathrm{~g}, 0.0178 \mathrm{mmol})$ in $\mathrm{C}_{6} \mathrm{D}_{6}(0.6 \mathrm{~mL})$. The tube was heated at $70{ }^{\circ} \mathrm{C}$ for $16 \mathrm{~h}$, after which time the ${ }^{1} \mathrm{H}$ NMR spectrum indicated quantitative conversion to $\mathbf{2 0 .}$

$\mathbf{T i}\left(\mathbf{N}_{2} \mathbf{N}^{\mathbf{p y}}\right)\left\{\mathbf{N B}\left(\mathrm{NAr}^{\prime} \mathbf{C H}\right)_{2}\right\}(\mathbf{p y})$ (21). To a Schlenk flask containing $\mathrm{Ti}\left\{\mathrm{NB}\left(\mathrm{NAr}^{\prime} \mathrm{CH}\right)_{2}\right\} \mathrm{Cl}_{2}(\mathrm{py})_{3}(\mathbf{3}$, $1.50 \mathrm{~g}, 1.98 \mathrm{mmol})$ and $\mathrm{Li}_{2} \mathrm{~N}_{2} \mathrm{~N}^{\mathrm{py}}(0.668 \mathrm{~g}, 2.08 \mathrm{mmol})$ was added toluene $(25 \mathrm{~mL})$ at $-78{ }^{\circ} \mathrm{C}$. The mixture was allowed to warm to room temperature and then stirred for $3 \mathrm{~h}$, after which it had become a dark orange suspension. The volatiles were removed under reduced pressure, and the product extracted into hexane $(2 \times 20 \mathrm{~mL})$. The solvent was removed from the combined extracts under reduced pressure, resulting in $\mathbf{2 1}$ as an orange solid, which was dried in vacuo. Yield: $1.38 \mathrm{~g}$ (83\%). Diffraction-quality crystals were grown from a concentrated pentane solution cooled to $5{ }^{\circ} \mathrm{C}$. ${ }^{1} \mathrm{H}$ NMR ( $\left.{ }_{6} \mathrm{D}_{6}, 400.1 \mathrm{MHz}\right): \delta 8.70\left(2 \mathrm{H}, \mathrm{d},{ }^{3} J=4.6 \mathrm{~Hz}, 2,6-p y\right), 8.05\left(1 \mathrm{H}\right.$, br. m, 6- $\left.{ }_{5} \mathrm{H}_{4} \mathrm{~N}\right), 7.18(6$ $\mathrm{H}$, overlapping $2 \times \mathrm{m}, m$ - and $\left.p-\mathrm{C}_{6} \underline{\mathrm{H}}_{3}{ }_{3} \operatorname{Pr}_{2}\right), 7.03(1 \mathrm{H}, \mathrm{m}, 4-\mathrm{py}), 6.88\left(1 \mathrm{H}, \mathrm{m}, 4-\mathrm{C}_{5} \mathrm{H}_{4} \mathrm{~N}\right), 6.70(1 \mathrm{H}, \mathrm{d}$, $\left.{ }^{3} J=8.0 \mathrm{~Hz}, 3-\mathrm{C}_{5} \mathrm{H}_{4} \mathrm{~N}\right), 6.53\left(3 \mathrm{H}, \mathrm{m}\right.$, overlapping 3,5-py and 5- $\left.\mathrm{C}_{5} \mathrm{H}_{4} \mathrm{~N}\right), 5.89(2 \mathrm{H}, \mathrm{s}, \mathrm{NCH}), 3.90(4$ H, sept., $\left.{ }^{3} J=6.9 \mathrm{~Hz}, \mathrm{C} \underline{\mathrm{HMeMe}}\right), 3.36\left(2 \mathrm{H}, \mathrm{d},{ }^{2} J=12.4 \mathrm{~Hz}, \mathrm{NCH}_{2}\right), 3.09\left(2 \mathrm{H}, \mathrm{d},{ }^{2} J=12.4 \mathrm{~Hz}\right.$, 
$\left.\mathrm{NCH}_{2}\right), 1.40\left(12 \mathrm{H}, \mathrm{d},{ }^{3} \mathrm{~J}=6.9 \mathrm{~Hz}, \mathrm{CHMeMe}\right), 1.34\left(12 \mathrm{H}, \mathrm{d},{ }^{3} \mathrm{~J}=6.9 \mathrm{~Hz}, \mathrm{CHMeMe}\right), 0.94(3 \mathrm{H}, \mathrm{s}$, $\mathrm{CMe}), 0.09\left(18 \mathrm{H}, \mathrm{s}, \mathrm{SiMe}_{3}\right)$ ppm. ${ }^{13} \mathrm{C}\left\{{ }^{1} \mathrm{H}\right\} \mathrm{NMR}\left(\mathrm{C}_{6} \mathrm{D}_{6}, 100.6 \mathrm{MHz}\right): \delta 159.6\left(2-\mathrm{C}_{5} \mathrm{H}_{4} \mathrm{~N}\right), 150.9$ (2,6-py), $150.6\left(6-\mathrm{C}_{5} \mathrm{H}_{4} \mathrm{~N}\right), 147.6\left(o-\underline{\mathrm{C}}_{6} \mathrm{H}_{3}{ }^{\mathrm{i}} \mathrm{Pr}_{2}\right), 143.2\left(i-\underline{\mathrm{C}}_{6} \mathrm{H}_{3}{ }^{\mathrm{i}} \mathrm{Pr}_{2}\right), 137.1$ (4-py), $136.6\left(4-\mathrm{C}_{5} \mathrm{H}_{4} \mathrm{~N}\right)$, $126.6\left(p-\underline{\mathrm{C}}_{6} \mathrm{H}_{3}{ }^{\mathrm{i}} \mathrm{Pr}_{2}\right), 123.7\left(m-\underline{\mathrm{C}}_{6} \mathrm{H}_{3}{ }^{\mathrm{i}} \mathrm{Pr}_{2}\right), 123.3$ (3,5-py), $121.2\left(5-\mathrm{C}_{5} \mathrm{H}_{4} \mathrm{~N}\right), 119.9\left(3-\mathrm{C}_{5} \mathrm{H}_{4} \mathrm{~N}\right), 117.3$

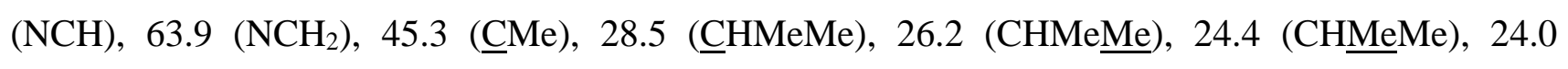
$(\mathrm{CMe}), 1.0\left(\mathrm{SiMe}_{3}\right) \mathrm{ppm} .{ }^{11} \mathrm{~B}\left\{{ }^{1} \mathrm{H}\right\} \mathrm{NMR}\left(\mathrm{C}_{6} \mathrm{D}_{6}, 128.4 \mathrm{MHz}\right): \delta 14.5 \mathrm{ppm}$. IR (NaCl plates, Nujol mull, $\left.\mathrm{cm}^{-1}\right): 2781$ (w), 1601 (s), 1570 (m), 1240 (m), 1209 (w), 1177 (w), 1113 (m), 1053 (s), 994 (m), $952(\mathrm{w}), 892(\mathrm{~m}), 875(\mathrm{w}), 829(\mathrm{~m}), 775(\mathrm{w}), 762(\mathrm{~m}), 701(\mathrm{~m}), 668(\mathrm{~m}), 643(\mathrm{~m}), 560(\mathrm{w})$. EIMS: $m / z=756[M-p y]^{+}(38 \%)$. Anal. found (calcd. for $\mathrm{C}_{46} \mathrm{H}_{70} \mathrm{BN}_{7} \mathrm{Si}_{2} \mathrm{Ti}$ ): C, 65.95 (66.09); H, 8.58 (8.44); N, $11.88(11.73) \%$.

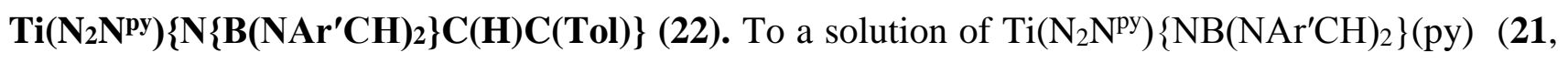
$0.300 \mathrm{~g}, 0.359 \mathrm{mmol})$ in toluene $(10 \mathrm{~mL})$ was added TolCCH $(46 \mu \mathrm{L}, 0.359 \mathrm{mmol})$ at RT. The mixture was heated to $60{ }^{\circ} \mathrm{C}$ and stirred for $2 \mathrm{~h}$, then the volatiles removed under reduced pressure. The dark brown waxy solid was then dissolved in hexane $(10 \mathrm{~mL})$, concentrated to $\sim 5 \mathrm{~mL}$ and cooled to $5{ }^{\circ} \mathrm{C}$, resulting in deep brown, diffraction-quality crystals of 22 . Yield: $0.076 \mathrm{~g}(24 \%) .{ }^{1} \mathrm{H}$ NMR $\left(\mathrm{C}_{6} \mathrm{D}_{6}, 400.1 \mathrm{MHz}\right): \delta 9.56(1 \mathrm{H}, \mathrm{s}, \mathrm{TiC}=\mathrm{CH}), 9.07\left(1 \mathrm{H}, \mathrm{d},{ }^{3} J=4.3 \mathrm{~Hz}, 6-\mathrm{C}_{5} \mathrm{H}_{4} \mathrm{~N}\right), 7.35(2 \mathrm{H}$, $\left.\mathrm{d},{ }^{3} J=8.0 \mathrm{~Hz}, m-\mathrm{C}_{6} \underline{\mathrm{H}}_{4} \mathrm{Me}\right), 7.20-7.09(9 \mathrm{H}, 5 \times \mathrm{m}$ overlapping with residual protio solvent resonance, $m$ - and $p-\mathrm{C}_{6} \underline{\mathrm{H}}_{3}{ }^{\mathrm{i}} \mathrm{Pr}_{2}$ and $\left.3,4,5-\mathrm{C}_{5} \mathrm{H}_{4} \mathrm{~N}\right), 6.88\left(2 \mathrm{H}, \mathrm{d},{ }^{3} J=8.0 \mathrm{~Hz}, o-\mathrm{C}_{6} \underline{\mathrm{H}}_{4} \mathrm{Me}\right), 6.21(2 \mathrm{H}, \mathrm{s}$, $\mathrm{NCH}), 4.07\left(2 \mathrm{H}, \mathrm{d},{ }^{2} J=12.3 \mathrm{~Hz}, \mathrm{NCH}_{2}\right), 3.55$ (4 H, sept., $\left.{ }^{3} J=6.9 \mathrm{~Hz}, \mathrm{C} \underline{\mathrm{HMeMe}}\right), 3.22\left(2 \mathrm{H}, \mathrm{d},{ }^{2} J\right.$ $\left.=12.3 \mathrm{~Hz}, \mathrm{NCH}_{2}\right), 2.18\left(3 \mathrm{H}, \mathrm{s}, \mathrm{C}_{6} \mathrm{H}_{4} \underline{\mathrm{Me}}\right), 1.29\left(12 \mathrm{H}, \mathrm{d},{ }^{3} J=6.9 \mathrm{~Hz}, \mathrm{CHMeMe}\right), 1.15(3 \mathrm{H}, \mathrm{s}$, $\mathrm{CMe}), 1.12\left(12 \mathrm{H}, \mathrm{d},{ }^{3} J=6.9 \mathrm{~Hz}, \mathrm{CHMeMe}\right),-0.26\left(18 \mathrm{H}, \mathrm{s}, \mathrm{SiMe}_{3}\right) \mathrm{ppm} .{ }^{13} \mathrm{C}\left\{{ }^{1} \mathrm{H}\right\} \mathrm{NMR}\left(\mathrm{C}_{6} \mathrm{D}_{6}\right.$, $125.7 \mathrm{MHz}): \delta 210.6(\mathrm{Ti} \underline{\mathrm{C}}=\mathrm{CH}), 162.0\left(2-\mathrm{C}_{5} \mathrm{H}_{4} \mathrm{~N}\right), 154.9(\mathrm{TiC}=\underline{\mathrm{CH}}), 146.9\left(o-\underline{\mathrm{C}}_{6} \mathrm{H}_{3}{ }^{\mathrm{i}} \operatorname{Pr}_{2}\right), 146.7$ (6$\left.\mathrm{C}_{5} \mathrm{H}_{4} \mathrm{~N}\right), 143.0\left(5-\mathrm{C}_{5} \mathrm{H}_{4} \mathrm{~N}\right), 140.0\left(i-\underline{\mathrm{C}}_{6} \mathrm{H}_{3}{ }^{\mathrm{i}} \mathrm{Pr}_{2}\right), 138.5\left(4-\mathrm{C}_{5} \mathrm{H}_{4} \mathrm{~N}\right), 132.1\left(p-\underline{\mathrm{C}}_{6} \mathrm{H}_{4} \mathrm{Me}\right), 129.2(p-$ $\left.\underline{\mathrm{C}}_{6} \mathrm{H}_{3}{ }^{\mathrm{i}} \mathrm{Pr}_{2}\right), 127.6\left(3-\mathrm{C}_{5} \mathrm{H}_{4} \mathrm{~N}\right), 124.9\left(m-\underline{\mathrm{C}}_{6} \mathrm{H}_{4} \mathrm{Me}\right), 123.9\left(m-\underline{\mathrm{C}}_{6} \mathrm{H}_{3}{ }^{\mathrm{i}} \mathrm{Pr}_{2}\right), 121.1\left(i-\underline{\mathrm{C}}_{6} \mathrm{H}_{4} \mathrm{Me}\right), 120.0$ (o$\left.\underline{\mathrm{C}}_{6} \mathrm{H}_{4} \mathrm{Me}\right), 119.2(\mathrm{NCH}), 63.4\left(\mathrm{NCH}_{2}\right), 51.0$ (미), 29.0 (ㄷHMeMe), 26.5 (CHMeMe), 23.8

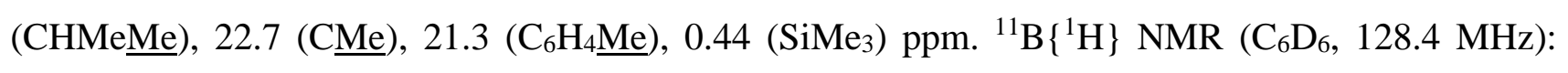
25.5 ppm. IR ( $\mathrm{NaCl}$ plates, Nujol mull, $\left.\mathrm{cm}^{-1}\right): 3437$ (w), $1656(\mathrm{~m}), 1601(\mathrm{w}), 1587$ (m), $1571(\mathrm{w})$, 1403 (s), 1246 (s), 1179 (w), 1159 (w), 1140 (w), 1115 (w), 1059 (m), 915 (m), 856 (s), 837 (s), 806 (m), $780(\mathrm{w}), 762(\mathrm{~m}), 683(\mathrm{w}), 645(\mathrm{~m})$. EI-MS: m/z = 519 [TolC $\left.(\mathrm{H}) \mathrm{C}(\mathrm{H}) \mathrm{N}(\mathrm{H}) \mathrm{B}\left(\mathrm{NAr}{ }^{\prime} \mathrm{CH}\right)_{2}\right]^{+}(8 \%)$. Anal. found (calcd. for $\mathrm{C}_{50} \mathrm{H}_{73} \mathrm{BN}_{6} \mathrm{Si}_{2} \mathrm{Ti}$ ): C, 68.66 (68.79); H, 8.52 (8.43); N, 9.51 (9.63)\%.

$\operatorname{Ti}\left(\mathbf{N}_{2} \mathbf{N}^{p y}\right)\left\{\mathbf{N H B}\left(\mathbf{N A r}^{\prime} \mathbf{C H}\right)_{2}\right\}\left(\mathbf{C C A r}^{\mathrm{F}}\right)$ (23). To a solution of $\operatorname{Ti}\left(\mathrm{N}_{2} \mathrm{~N}^{\mathrm{py}}\right)\left\{\mathrm{NB}\left(\mathrm{NAr}{ }^{\prime} \mathrm{CH}\right)_{2}\right\}(\mathrm{py})$ (21, $0.300 \mathrm{~g}, 0.359 \mathrm{mmol})$ in toluene $(10 \mathrm{~mL})$ was added $\operatorname{Ar}^{\mathrm{F}} \mathrm{CCH}(47.5 \mu \mathrm{L}, 0.359 \mathrm{mmol})$ at $\mathrm{RT}$, then the 
mixture left to stir for $24 \mathrm{~h}$, resulting in a deep red-brown solution. The volatiles were then removed under reduced pressure, and the crude product dissolved in hexane $(10 \mathrm{~mL})$. This solution was concentrated to $\sim 5 \mathrm{~mL}$ and cooled to $5{ }^{\circ} \mathrm{C}$, resulting in orange, diffraction-quality crystals of 23 . Yield: $0.075 \mathrm{~g}(22 \%) .{ }^{1} \mathrm{H}$ NMR $\left(\mathrm{C}_{6} \mathrm{D}_{6}, 400.1 \mathrm{MHz}\right): \delta 9.11\left(1 \mathrm{H}, \mathrm{d},{ }^{3} J=5.3 \mathrm{~Hz}, 6-\mathrm{C}_{5} \mathrm{H}_{4} \mathrm{~N}\right), 7.25-$ $7.18\left(6 \mathrm{H}\right.$, overlapping $2 \times \mathrm{m}, m-$ and $\left.p-\mathrm{C}_{6} \underline{\mathrm{H}}_{3}{ }^{\mathrm{i}} \mathrm{Pr}_{2}\right), 7.13\left(1 \mathrm{H}, \mathrm{m}, 4-\mathrm{C}_{5} \mathrm{H}_{4} \mathrm{~N}\right), 6.88\left(1 \mathrm{H}, \mathrm{m}, 5-\mathrm{C}_{5} \mathrm{H}_{4} \mathrm{~N}\right)$, $6.82\left(1 \mathrm{H}, \mathrm{d},{ }^{3} J=7.9 \mathrm{~Hz}, 3-\mathrm{C}_{5} \mathrm{H}_{4} \mathrm{~N}\right), 6.08\left(1 \mathrm{H}, \mathrm{s}, \mathrm{NHB}\left(\mathrm{NAr}^{\prime} \mathrm{CH}\right)_{2}\right), 6.01(2 \mathrm{H}, \mathrm{s}, \mathrm{NCH}), 3.99(2 \mathrm{H}, \mathrm{d}$, $\left.{ }^{2} J=12.8 \mathrm{~Hz}, \mathrm{NCH}_{2}\right), 3.44$ (4 H, sept., $\left.{ }^{3} J=6.9 \mathrm{~Hz}, \mathrm{CHMeMe}\right), 3.16\left(2 \mathrm{H}, \mathrm{d},{ }^{2} J=12.8 \mathrm{~Hz}, \mathrm{NCH}_{2}\right)$,

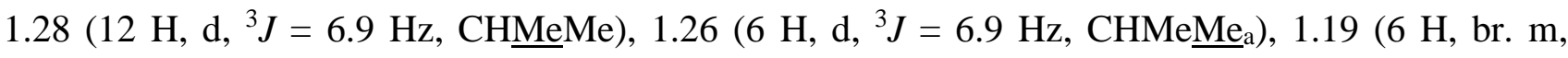

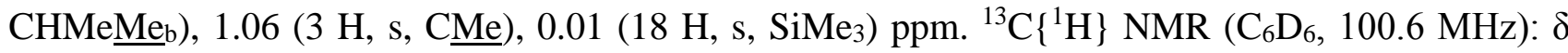
$162.0\left(2-\mathrm{C}_{5} \mathrm{H}_{4} \mathrm{~N}\right), 161.1\left(\mathrm{TiCCAr}{ }^{\mathrm{F}}\right), 148.7\left(\mathrm{~m}, o-\mathrm{C}_{6} \mathrm{~F}_{5}\right), 147.6\left(o_{\mathrm{a}}-\underline{\mathrm{C}}_{6} \mathrm{H}_{3}{ }^{\mathrm{i}} \mathrm{Pr}_{2}\right), 147.5\left(6-\mathrm{C}_{5} \mathrm{H}_{4} \mathrm{~N}\right), 147.2$ $\left(o{ }^{\mathrm{a}}-\underline{\mathrm{C}}_{6} \mathrm{H}_{3}{ }^{\mathrm{i}} \mathrm{Pr}_{2}\right), 139.7\left(i-\underline{\mathrm{C}}_{6} \mathrm{H}_{3}{ }^{\mathrm{i}} \mathrm{Pr}_{2}\right), 138.8\left(\mathrm{~m}, p-\mathrm{C}_{6} \mathrm{~F}_{5}\right), 138.5\left(4-\mathrm{C}_{5} \mathrm{H}_{4} \mathrm{~N}\right), 136.9\left(\mathrm{~m}, m-\mathrm{C}_{6} \mathrm{~F}_{5}\right), 127.6(p-$ $\left.\underline{\mathrm{C}}_{6} \mathrm{H}_{3}{ }^{\mathrm{i}} \mathrm{Pr}_{2}\right), 124.3\left(m_{\mathrm{a}}-\underline{\mathrm{C}}_{6} \mathrm{H}_{3}{ }^{\mathrm{i}} \operatorname{Pr}_{2}\right), 123.9\left(m_{\mathrm{a}}-\underline{\mathrm{C}}_{6} \mathrm{H}_{3}{ }^{\mathrm{i}} \operatorname{Pr}_{2}\right), 121.2\left(5-\mathrm{C}_{5} \mathrm{H}_{4} \mathrm{~N}\right), 119.5\left(3-\mathrm{C}_{5} \mathrm{H}_{4} \mathrm{~N}\right), 118.0$ $(\mathrm{NCH}), 103.6\left(\mathrm{td},{ }^{2} J_{\mathrm{C}-\mathrm{F}}=19.0 \mathrm{~Hz},{ }^{3} J_{\mathrm{C}-\mathrm{F}}=3.7 \mathrm{~Hz}, i-\mathrm{C}_{6} \mathrm{~F}_{5}\right), 87.6\left(\mathrm{TiC}{ }^{2}{ }^{\mathrm{F}}\right), 64.5\left(\mathrm{NCH}_{2}\right), 49.7$

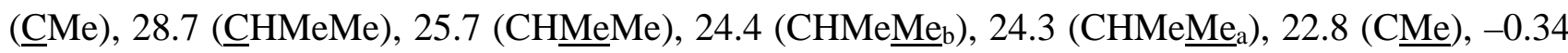
$\left(\mathrm{SiMe}_{3}\right)$ ppm. ${ }^{11} \mathrm{~B}\left\{{ }^{1} \mathrm{H}\right\}$ NMR $\left(\mathrm{C}_{6} \mathrm{D}_{6}, 128.4 \mathrm{MHz}\right): 23.0 \mathrm{ppm} .{ }^{19} \mathrm{~F}\left\{{ }^{1} \mathrm{H}\right\}$ NMR $\left(\mathrm{C}_{6} \mathrm{D}_{6}, 376.5 \mathrm{MHz}\right)$ :$138.8\left(2 \mathrm{~F}, \mathrm{~m}, o-\mathrm{C}_{6} \mathrm{~F}_{5}\right),-159.8\left(1 \mathrm{~F}, \mathrm{t},{ }^{3} \mathrm{~J}=21.4 \mathrm{~Hz}, p-\mathrm{C}_{6} \mathrm{~F}_{5}\right),-164.7\left(2 \mathrm{~F}, \mathrm{~m}, m-\mathrm{C}_{6} \mathrm{~F}_{5}\right) \mathrm{ppm}$. IR $(\mathrm{NaCl}$

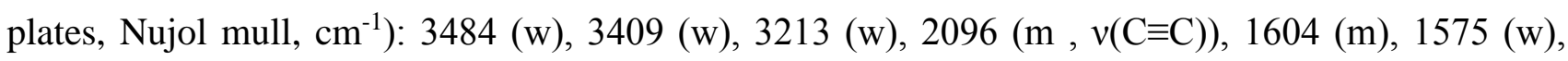
1512 (s), 1492 (s), 1354 (s), 1326 (w), 1289 (w), 1275 (w), 1184 (w), 1141 (m), 1112 (m), 1059 (m), 1047 (m), 989 (s), 958 (s), 913 (s), 890 (w), 839 (s), 808 (m), 754 (m), 688 (w), 657 (m), 645 (w), $611(\mathrm{~m})$. EI-MS: $m / z=595\left[\mathrm{Ar}^{\mathrm{F}} \mathrm{C}(\mathrm{H}) \mathrm{C}(\mathrm{H}) \mathrm{N}(\mathrm{H}) \mathrm{B}\left(\mathrm{NAr}{ }^{\prime} \mathrm{CH}\right)_{2}\right]^{+}(56 \%)$. Anal. found (calcd. for $\left.\mathrm{C}_{49} \mathrm{H}_{66} \mathrm{BF}_{5} \mathrm{~N}_{6} \mathrm{Si}_{2} \mathrm{Ti}\right): \mathrm{C}, 61.89$ (62.02); H, 7.13 (7.01); N, 9.01 (8.86)\%.

Kinetic isotope effect measurements for the reaction of $\mathrm{Ti}_{2}\left(\mathrm{~N}_{2} \mathrm{~N}^{\mathrm{py}}\right)\left\{\mathrm{NB}\left(\mathrm{NAr} \mathbf{C}^{\prime} \mathrm{CH}\right)_{2}\right\}$ (21) with $\mathrm{Ar}^{\mathrm{F}} \mathrm{CCH}$ to form $\mathbf{T i}\left(\mathbf{N}_{2} \mathbf{N}^{\mathrm{py}}\right)\left\{\mathbf{N H B}\left(\mathbf{N A r} \mathbf{C H}^{\prime} \mathbf{C}\right)_{2}\left(\mathbf{C C A r}^{\mathrm{F}}\right)\right.$ (23). To a $\mathrm{C}_{6} \mathrm{D}_{6}$ solution $(0.6 \mathrm{~mL})$ of $\mathrm{Ti}\left(\mathrm{N}_{2} \mathrm{~N}^{\mathrm{py}}\right)\left\{\mathrm{NB}\left(\mathrm{NAr}{ }^{\prime} \mathrm{CH}\right)_{2}\right\}$ (py) (21, $\left.0.0150 \mathrm{~g}, 0.0179 \mathrm{mmol}\right)$ in an NMR tube equipped with a $\mathrm{J}$. Youngs valve was added $\operatorname{Ar}^{\mathrm{F}} \mathrm{CCH}$ or $\operatorname{Ar}^{\mathrm{F}} \mathrm{CCD}(2.4 \mu \mathrm{L}, 0.0179 \mathrm{mmol})$ via microsyringe. The NMR tube was transferred to a temperature-regulated $(298 \mathrm{~K})$ probe, and the reaction monitored by ${ }^{1} \mathrm{H}$ NMR spectroscopy using an array which recorded spectra (4 scans) every 120 s. The formation of 23 (or 23-d $\mathbf{d}_{1}$ ) was calculated by measuring the integral of an $\mathrm{NCH}_{2}$ resonance. Initial rate constants were obtained from plots of [23] vs time.

Competition reaction of $\mathrm{Ti}\left(\mathrm{N}_{2} \mathrm{~N}^{\mathrm{py}}\right)\left\{\mathrm{NB}\left(\mathrm{NAr} \mathbf{r}^{\prime} \mathrm{CH}\right)_{2}\right\}(\mathbf{p y})$ (21) with $\mathrm{Ar}^{\mathrm{F}} \mathrm{CCH}$ and $\operatorname{Ar}^{\mathrm{F}} \mathrm{CCD}$. To a $\mathrm{C}_{6} \mathrm{D}_{6}$ solution $(0.3 \mathrm{~mL})$ of $\mathrm{Ti}\left(\mathrm{N}_{2} \mathrm{~N}^{\mathrm{py}}\right)\left\{\mathrm{NB}\left(\mathrm{NAr} \mathrm{CH}_{2}\right\}(\right.$ py) $(\mathbf{2 1}, 0.0100 \mathrm{~g}, 0.0120 \mathrm{mmol})$ in an $\mathrm{NMR}$ tube fitted with a J. Youngs valve was added a $\mathrm{C}_{6} \mathrm{D}_{6}$ solution $(0.3 \mathrm{~mL})$ of $\mathrm{Ar}^{\mathrm{F}} \mathrm{CCH}(7.9 \mu \mathrm{L}, 0.0598$ 
mmol) and $\operatorname{Ar}^{\mathrm{F} C C D}(8.0 \mu \mathrm{L}, 0.0598 \mathrm{mmol})$. The mixture was then left at RT for $16 \mathrm{~h}$, with ${ }^{1} \mathrm{H}$ NMR integration showing the products to be $87 \% \mathbf{2 3}$ and $13 \% \mathbf{2 3}-\boldsymbol{d}_{\mathbf{1}}$.

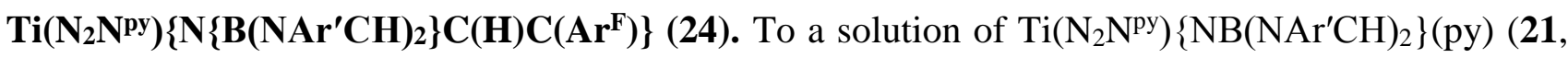
$0.267 \mathrm{~g}, 0.319 \mathrm{mmol})$ in toluene $(10 \mathrm{~mL})$ was added $\operatorname{Ar}^{\mathrm{F}} \mathrm{CCH}(42.5 \mu \mathrm{L}, 0.359 \mathrm{mmol})$ at $\mathrm{RT}$, then the mixture heated to $60{ }^{\circ} \mathrm{C}$ and stirred for $40 \mathrm{~h}$, resulting in a deep brown solution. The volatiles were then removed under reduced pressure, and $\mathbf{2 4}$ isolated as a deep brown, waxy solid. Yield: $0.195 \mathrm{~g}$ (64\%). ${ }^{1} \mathrm{H}$ NMR $\left(\mathrm{C}_{6} \mathrm{D}_{6}, 500.3 \mathrm{MHz}\right): \delta 10.05(1 \mathrm{H}, \mathrm{s}, \mathrm{TiC}=\mathrm{CH}), 9.04\left(1 \mathrm{H}, \mathrm{d},{ }^{3} J=5.2 \mathrm{~Hz}, 6-\mathrm{C}_{5} \mathrm{H}_{4} \mathrm{~N}\right)$, 7.25 - $7.17\left(6 \mathrm{H}\right.$, overlapping $2 \times \mathrm{m}, m$ - and $\left.p-\mathrm{C}_{6} \underline{\mathrm{H}}_{3}{ }^{\mathrm{i}} \operatorname{Pr}_{2}\right), 7.10\left(1 \mathrm{H}, \mathrm{m}, 4-\mathrm{C}_{5} \mathrm{H}_{4} \mathrm{~N}\right), 6.89(1 \mathrm{H}, \mathrm{m}, 5-$ $\left.\mathrm{C}_{5} \mathrm{H}_{4} \mathrm{~N}\right), 6.85\left(1 \mathrm{H}, \mathrm{d},{ }^{3} \mathrm{~J}=8.1 \mathrm{~Hz}, 3-\mathrm{C}_{5} \mathrm{H}_{4} \mathrm{~N}\right), 6.20(2 \mathrm{H}, \mathrm{s}, \mathrm{NCH}), 4.09\left(2 \mathrm{H}, \mathrm{d},{ }^{2} J=12.4 \mathrm{~Hz}, \mathrm{NCH}_{2}\right)$, 3.46 (4 H, sept., $\left.{ }^{3} J=6.9 \mathrm{~Hz}, \mathrm{CHMeMe}\right), 3.12\left(2 \mathrm{H}, \mathrm{d},{ }^{2} J=6.9 \mathrm{~Hz}, \mathrm{NCH}_{2}\right), 1.28\left(6 \mathrm{H}, \mathrm{d},{ }^{3} J=6.9 \mathrm{~Hz}\right.$, CHMe $\left.\underline{a M e}_{\mathrm{a}}\right), 1.26\left(12 \mathrm{H}, \mathrm{d},{ }^{3} \mathrm{~J}=6.9 \mathrm{~Hz}, \mathrm{CHMeMe}\right), 1.10(3 \mathrm{H}, \mathrm{s}, \mathrm{CMe}), 1.08$ (6 H, br. m, $\left.\mathrm{CHMe}{ }_{b} \mathrm{Me}\right),-0.28\left(18 \mathrm{H}, \mathrm{s}, \mathrm{SiMe}_{3}\right) \mathrm{ppm} .{ }^{13} \mathrm{C}\left\{{ }^{1} \mathrm{H}\right\}$ NMR $\left(\mathrm{C}_{6} \mathrm{D}_{6}, 125.7 \mathrm{MHz}\right): \delta 177.9(\mathrm{TiC}=\mathrm{CH})$, $161.4(\mathrm{TiC}=\underline{\mathrm{CH}}), 161.2\left(2-\mathrm{C}_{5} \mathrm{H}_{4} \mathrm{~N}\right), 147.6\left(o-\underline{\mathrm{C}}_{6} \mathrm{H}_{3}{ }^{\mathrm{i}} \mathrm{Pr}_{2}\right), 146.4\left(6-\mathrm{C}_{5} \mathrm{H}_{4} \mathrm{~N}\right), 142.9\left(\mathrm{~m}, o-\mathrm{C}_{6} \mathrm{~F}_{5}\right), 140.9$ (m, $\left.m-\mathrm{C}_{6} \mathrm{~F}_{5}\right), 139.5\left(i-\underline{\mathrm{C}}_{6} \mathrm{H}_{3}{ }^{\mathrm{i}} \mathrm{Pr}_{2}\right), 138.9\left(4-\mathrm{C}_{5} \mathrm{H}_{4} \mathrm{~N}\right), 137.4\left(\mathrm{~m}, p-\mathrm{C}_{6} \mathrm{~F}_{5}\right), 127.6\left(p-\underline{\mathrm{C}}_{6} \mathrm{H}_{3}{ }^{\mathrm{i}} \mathrm{Pr}_{2}\right), 124.0(m-$ $\left.\underline{\mathrm{C}}_{6} \mathrm{H}_{3}{ }^{\mathrm{i}} \mathrm{Pr}_{2}\right), 121.4\left(5-\mathrm{C}_{5} \mathrm{H}_{4} \mathrm{~N}\right), 120.2\left(3-\mathrm{C}_{5} \mathrm{H}_{4} \mathrm{~N}\right), 119.5\left(\mathrm{td},{ }^{2} J_{\mathrm{C}-\mathrm{F}}=14.5 \mathrm{~Hz},{ }^{3} J_{\mathrm{C}-\mathrm{F}}=4.5 \mathrm{~Hz}, i-\mathrm{C}_{6} \mathrm{~F}_{5}\right)$,

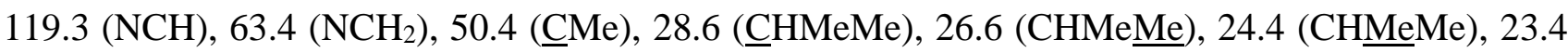
$(\mathrm{CMe}), 0.01\left(\mathrm{SiMe}_{3}\right)$ ppm. ${ }^{11} \mathrm{~B}\left\{{ }^{1} \mathrm{H}\right\}$ NMR $\left(\mathrm{C}_{6} \mathrm{D}_{6}, 128.4 \mathrm{MHz}\right): 25.0 \mathrm{ppm} .{ }^{19} \mathrm{~F}\left\{{ }^{1} \mathrm{H}\right\} \mathrm{NMR}\left(\mathrm{C}_{6} \mathrm{D}_{6}\right.$, $376.5 \mathrm{MHz}):-141.5\left(2 \mathrm{~F}, \mathrm{~m}, o-\mathrm{C}_{6} \mathrm{~F}_{5}\right),-166.9\left(2 \mathrm{~F}, \mathrm{~m}, m-\mathrm{C}_{6} \mathrm{~F}_{5}\right),-168.5\left(1 \mathrm{~F}, \mathrm{tt},{ }^{3} J=21.6 \mathrm{~Hz},{ }^{4} J 4.1\right.$ $\left.\mathrm{Hz}, p-\mathrm{C}_{6} \mathrm{~F}_{5}\right)$ ppm. IR ( $\mathrm{NaCl}$ plates, Nujol mull, $\mathrm{cm}^{-1}$ ): 3522 (w), 3436 (w), 3391 (w), 1931 (w), 1865 (w), 1655 (m), 1638 (m), 1602 (m), 1587 (s), 1506 (s), 1327 (m), 1209 (m), 1180 (w), 1116 (m), 1059 (s), 959 (s), 913 (s), 856 (s), 780 (m), 758 (s), 675 (m), 646 (m), 604 (m). EI-MS: m/z = 595 $\left[\mathrm{Ar}^{\mathrm{F}} \mathrm{C}(\mathrm{H}) \mathrm{C}(\mathrm{H}) \mathrm{N}(\mathrm{H}) \mathrm{B}\left(\mathrm{NAr}{ }^{\prime} \mathrm{CH}\right)_{2}\right]^{+}(4 \%)$. Anal. found (calcd. for $\mathrm{C}_{49} \mathrm{H}_{66} \mathrm{BF}_{5} \mathrm{~N}_{6} \mathrm{Si}_{2} \mathrm{Ti}$ ): $\mathrm{C}, 61.88$ (62.02); H, 7.10 (7.01); N, 9.02 (8.86)\%.

NMR tube scale synthesis of $\operatorname{Ti}\left(\mathbf{N}_{2} \mathbf{N}^{p y}\right)\left\{\mathbf{N}\left\{\mathbf{B}\left(\mathbf{N A r} \mathbf{r}^{\prime} \mathbf{C H}\right)_{2}\right\} \mathbf{C}(\mathbf{H}) \mathbf{C}\left(\mathbf{A r}^{\mathrm{F}}\right)\right\}$ (24). To an NMR tube

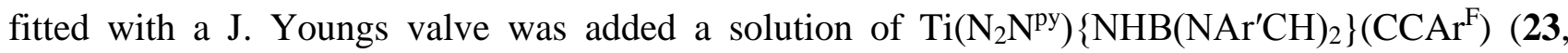
$0.0150 \mathrm{~g}, 0.0158 \mathrm{mmol})$ in $\mathrm{C}_{6} \mathrm{D}_{6}(0.6 \mathrm{~mL})$. The tube was heated at $70{ }^{\circ} \mathrm{C}$ for $18 \mathrm{~h}$, after which time the ${ }^{1} \mathrm{H}$ NMR spectrum indicated quantitative conversion to $\mathbf{2 4}$.

Kinetic isotope effect measurements for the conversion of $\left.\operatorname{Ti}^{\left(N_{2} N^{p y}\right.}\right)\left\{\mathrm{NHB}\left(\mathrm{NAr}^{\prime} \mathrm{CH}\right)_{2}\right\}\left(\mathrm{CCAr}^{\mathrm{F}}\right)$ (23) to $\mathbf{T i}\left(\mathbf{N}_{2} \mathbf{N}^{\mathrm{py}}\right)\left\{\mathbf{N}\left\{\mathbf{B}\left(\mathbf{N A r ^ { \prime }} \mathbf{C H}\right)_{2}\right\} \mathbf{C}(\mathbf{H}) \mathbf{C}\left(\mathbf{A} \mathbf{r}^{\mathbf{F}}\right)\right\} \quad(\mathbf{2 4}) . \quad \mathrm{A} \quad \mathrm{C}_{6} \mathrm{D}_{6}$ solution $\left(\begin{array}{llll}0.6 & \mathrm{~mL}) & \text { of }\end{array}\right.$ $\mathrm{Ti}\left(\mathrm{N}_{2} \mathrm{~N}^{\mathrm{py}}\right)\left\{\mathrm{NHB}\left(\mathrm{NAr}^{\prime} \mathrm{CH}\right)_{2}\right\}\left(\mathrm{CCAr}^{\mathrm{F}}\right)(\mathbf{2 3}, 0.0150 \mathrm{~g}, 0.0158 \mathrm{mmol})$ or $\mathbf{2 3}-\boldsymbol{d}_{\mathbf{1}}$ was transferred to an NMR tube equipped with a J. Youngs valve. A blank $\mathrm{C}_{6} \mathrm{D}_{6}$ sample was added to the NMR probe at RT, locked and shimmed, and the probe heated to $343 \mathrm{~K}$. After thermal equilibration, the 
experimental sample replaced the blank sample, the shimming was checked and an array was set up to record a ${ }^{1} \mathrm{H}$ NMR spectrum (4 scans) every $120 \mathrm{~s}$ (or every $600 \mathrm{~s}$ for $\mathbf{2 3}-\boldsymbol{d}_{\mathbf{1}}$ ). The decay of 23 was calculated by measuring the integral of an $\mathrm{NCH}_{2}$ doublet. First order rate constants were obtained from linear plots of $-\ln \left([\mathbf{2 3}] /[\mathbf{2 3}]_{0}\right)$ vs time.

X-ray data collection and processing procedures. Crystals were mounted on glass fibers using perfluoropolyether oil and cooled rapidly in a stream of cold $\mathrm{N}_{2}$ using an Oxford Cryosystems Cryostream unit. Diffraction data were measured using either an Enraf-Nonius KappaCCD or Agilent Technologies Supernova diffractometer using Mo- $K_{\alpha}$ or $\mathrm{Cu}-K_{\alpha}$ radiation, respectively. As appropriate, absorption and decay corrections were applied to the data and equivalent reflections merged. ${ }^{9}$ The structures were solved with SIR92 ${ }^{10}$ or Superflip, ${ }^{11}$ and further refinements and all other crystallographic calculations were performed using the CRYSTALS program suite. ${ }^{12}$ The CIF supporting informaton file contains specific details for the refinements of the individual structures. The CCDC codes for the structures in this paper are: CCDC $1575707-1575717$.

Computational Details. Geometry optimizations for all systems were performed with the Gaussian09 package (rev. D.01) ${ }^{13}$ at the B3PW91 level of hybrid density functional theory in the gas phase with all atoms represented by the def2-svp basis set. $^{14}$ For the sterically pruned systemsadditional single point calculations were carried out on the optimised geometry using the def2-tzvp basis set also in the gas phase, and dispersion was not accounted for in either the optimisation or single point calculations. For the sterically more realistic models dispersion was taken into account both at the optimization and single point stages using Grimme's d3(bj) corrections $^{15}$ as implemented in Gaussian09. In addition, the solvent (benzene) influence was taken into consideration in the single point calculations on the gas-phase optimised geometry with SCRF calculations within the SMD model. Gibbs free energies and enthalpies were obtained by summing the SCF or (where appropriate) SMD energy, the gas-phase Gibbs or enthalpy contribution at 298.15 $\mathrm{K}$ obtained from the geometry optimisations, and the $\mathrm{d} 3(\mathrm{bj})$ correction. NMR chemical shifts were computed using the GIAO method ${ }^{16}$ as implemented in Gaussian09 at the B3PW91 level. All atoms were described with the pcSseg-2 NMR basis sets of Jensen. ${ }^{17}$ The ${ }^{11} \mathrm{~B}$ NMR shifts are referenced with respect to the experimental value of $0.0 \mathrm{ppm}$ for $\mathrm{BF}_{3}\left(\mathrm{Et}_{2} \mathrm{O}\right)$. The Cartesian coordinates for the following structures are provided as a separate file. The total SCF and other energies (Hartree) are given below after each compound or transition state. 
Table S3. Total SCF Energies, thermal corrections to enthalpies, thermal corrections to Gibbs free energies (Hartree, at $298.15 \mathrm{~K}$ except for values in brackets which are for $343 \mathrm{~K}$ ) for the species depicted in Figure 8 of the main text or Figure S9 of the SI (gas phase reactions).

\begin{tabular}{|c|c|c|c|}
\hline Compound or transition state & Total SCF Energy & Thermal correction to $\mathrm{H}$ & Thermal correction to $G$ \\
\hline $\mathrm{PhCCH}$ & -308.38073 & 0.116805 & 0.07901 \\
\hline Pyridine & -248.27671 & $0.093032[0.095377]$ & $0.061356[0.056351]$ \\
\hline $\mathrm{Ar}^{\mathrm{F}} \mathrm{CCH}$ & -804.56489 & $0.080977[0.084088]$ & $0.032663[0.084088]$ \\
\hline $\operatorname{Ar}^{\mathrm{F}} \mathrm{CCD}$ & -804.56489 & $0.078311[0.081483]$ & $0.029601[0.022021]$ \\
\hline $1 Q$ & -2540.66384 & $0.499618[0.508877]$ & $0.39978[0.38411]$ \\
\hline $2 Q$ & -2292.35998 & $0.403413[0.411025]$ & $0.31716[0.30365]$ \\
\hline 3Q_Ar $\mathbf{r}^{\mathrm{F}}$ & -3096.95878 & $0.487667[0.498616]$ & $0.37285[0.35481]$ \\
\hline 3Q_ArF $-d_{1}$ & -3096.95878 & $0.484676[0.495714]$ & $0.36959[0.35143]$ \\
\hline 3Q_Ph & -2600.76993 & 0.523481 & 0.41858 \\
\hline 4Q_Ph & -2600.74784 & 0.522475 & 0.41657 \\
\hline 5Q_A $\mathbf{r}^{\mathrm{F}}$ & -3096.97777 & $0.488148[0.498957]$ & $0.37596[0.35833]$ \\
\hline 5Q_A $\mathbf{r}^{\mathrm{F}}-d_{1}$ & -3096.97777 & $0.485035[0.496028]$ & $0.37265[0.35502]$ \\
\hline 5Q_Ar ${ }^{\mathrm{F}} \_$2,1-addition (Markovnikov) & -3096.96477 & 0.487737 & 0.37470 \\
\hline 5Q_Ph & -2600.78435 & 0.523855 & 0.42190 \\
\hline 5Q_Ph_2,1-addition (Markovnikov) & -2600.77906 & 0.523657 & 0.42212 \\
\hline TS1_Ar ${ }^{F}$ & -3096.91467 & $0.482017[0.492872]$ & $0.36632[0.34815]$ \\
\hline TS1_Ar ${ }^{\mathrm{F}}-d_{1}$ & -3096.91467 & $0.480385[0.491325]$ & $0.36457[0.34631]$ \\
\hline TS1_Ph & -2600.72897 & 0.517934 & 0.41286 \\
\hline TS2_Ar ${ }^{F}$ & -3096.92298 & $0.485431[0.496254]$ & $0.37441[0.35695]$ \\
\hline
\end{tabular}


TS2_Ar ${ }^{\mathrm{F}}-d_{1}$

TS2_Ph

TS3_2Q_6Q_Ar ${ }^{\mathrm{F}}$

TS3_2Q_6Q_Ph

6Q_A ${ }^{F}$

6Q_Ph
$-3096.92298$

$-2600.73842$

$-3096.90635$

$-2600.72141$

$-3096.93225$

$-2600.74324$
0.482323 [0.493471]

0.521502

0.482583

0.518427

0.488928

0.524831
0.37110 [0.35372]

0.42005

0.36677

0.41308

0.37294

0.41995 
Table S4. Total SCF energies (E(B3PW91/SMD) + D3(bj), Hartree) and sums of SCF energy and thermal corrections to enthalpies or Gibbs free energies ( $\mathrm{kcal} \mathrm{mol}^{-1}$ at $298.15 \mathrm{~K}$ ) for the full experimental compounds.

\begin{tabular}{|c|c|c|c|}
\hline Compound & Total SCF Energy & $\begin{array}{l}\text { SCF Energy + Thermal } \\
\text { correction to G }\end{array}$ & $\begin{array}{l}\text { SCF Energy }+ \text { Thermal } \\
\text { correction to } \mathrm{H}\end{array}$ \\
\hline${ }^{\mathrm{t}} \mathrm{BuNH}_{2}$ & -213.83786 & -134110.4 & -134087.5 \\
\hline $\mathrm{XylNH}_{2}$ & -366.28228 & -229758.0 & -229731.2 \\
\hline $\mathrm{H}_{2} \mathrm{NB}\left(\mathrm{NAr}{ }^{\prime} \mathrm{CH}\right)_{2}$ & -1203.04531 & -754589.1 & -754527.1 \\
\hline Pyridine & -248.30109 & -155772.6 & -155752.2 \\
\hline \multicolumn{4}{|l|}{$\mathrm{PhCCH}$} \\
\hline $\mathrm{TolCCH}$ & -347.73761 & -218142.7 & -218117.4 \\
\hline $\mathrm{Ar}^{\mathrm{F}} \mathrm{CCH}$ & -804.59442 & -504870.1 & -504839.6 \\
\hline $\mathrm{Ar}^{\mathrm{F}} \mathrm{CCD}$ & -804.59442 & -504872.0 & -504841.3 \\
\hline $\mathrm{MeCCMe}$ & -155.99220 & -97850.9 & -97829.8 \\
\hline $\mathrm{PhCCMe}$ & -347.74565 & -218149.4 & -218121.3 \\
\hline${ }^{\mathrm{B}} \mathrm{BuNH}_{2} \_\mathrm{PhCCH}$ _amination (26) & -522.31858 & -327619.4 & -327586.4 \\
\hline Borylamine_TolCCH_amination (27a) & -1550.85337 & -972759.4 & -972685.3 \\
\hline${ }^{\mathrm{B}} \mathrm{BuNH}_{2}$ TolCCH_amination (27b) & -561.64129 & -352279.9 & -352243.6 \\
\hline $\mathrm{XylNH}_{2} \_\mathrm{TolCCH}$ _amination $(\mathbf{2 7 c})$ & -714.07979 & -447923.7 & -447883.4 \\
\hline 9 & -3479.80015 & -2182996.9 & -2182900.6 \\
\hline 9_Ar ${ }^{\mathrm{F}} \mathrm{CCH}$ _CH_activation & -4036.09471 & -2532094.7 & -2531988.6 \\
\hline 9_Ar ${ }^{\mathrm{F}} \mathrm{CCH}$ _cycloaddition & -4036.11985 & -2532107.0 & -2532004.2 \\
\hline 9_TolCCH_CH_activation & -3579.23021 & -2245364.1 & -2245260.8 \\
\hline 9_TolCCH_cycloaddition & -3579.25050 & -2245374.0 & -2245273.8 \\
\hline
\end{tabular}


10

10_Ar ${ }^{\mathrm{F}} \mathrm{CCH}$ _cycloaddition

10_TolCCH_cycloaddition

11

11_Ar ${ }^{\mathrm{F}} \mathrm{CH}$ _activation

11_A ${ }^{\mathrm{F}} \mathrm{CCH}$ _cycloadditon

11_TolCCH_CH_activation

11_TolCCH_cycloaddition

21

21_py-free

21_Ar ${ }^{\mathrm{F}} \mathrm{CCH}$ _CH_activation

21_Ar ${ }^{\mathrm{F}}$ CCD_CD_activation

21_Ar ${ }^{\mathrm{F}} \mathrm{CCH}$ _cycloaddition

21_MeCCMe

21_PhCCMe

21_TolCCH_CH_activation

21_TolCCH_cycloaddition

trans- $\mathrm{Ti}\left(\mathrm{N}_{2} \mathrm{~N}^{\mathrm{py}}\right)\left\{\mathrm{N}\left({ }^{ } \mathrm{Bu}\right) \mathrm{C}(\mathrm{H}) \mathrm{C}(\mathrm{Ph})\right\}($ (25)

$\operatorname{Ti}\left(\mathrm{N}_{2} \mathrm{~N}^{\mathrm{py}}\right)\left(\mathrm{N}^{\mathrm{t}} \mathrm{Bu}\right)(\mathrm{py})$
$-4116.90231$

$-4673.21769$

$-4216.35856$

$-2898.28855$

$-3454.58750$

$-3454.60858$

$-2997.71909$

$-2997.74380$

$-3632.25490$

$-3383.91930$

$-4188.55543$

$-4188.55543$

$-4188.57781$

$-3539.93946$

$-3731.70442$

$-3731.69194$

$-3731.70870$

$-2703.16808$

$-2643.02566$

$\begin{array}{ll}-2582866.2 & -2582763.0 \\ -2645247.0 & -2645139.8 \\ -2645247.7 & -2645139.0 \\ -1818112.1 & -1818022.9 \\ -2167211.9 & -2167113.1 \\ -2167223.2 & -2167126.9 \\ -1880481.8 & -1880384.1 \\ -1880493.7 & -1880398.4 \\ -2278653.8 & -2278552.6 \\ -2122876.2 & -2122781.2 \\ -2627755.1 & -2627643.9 \\ -2627757.2 & -2627645.8 \\ -2627766.2 & -2627657.2 \\ -2220726.2 & -2220625.6 \\ -2341028.9 & -2340924.0 \\ -2341022.5 & -2340918.1 \\ -2341022.5 & -2340918.1 \\ -1695896.9 & -1695825.1 \\ -1658170.0 & -1658101.2\end{array}$




\section{References for the Supporting Information}

1. Pangborn, A. B.; Giardello, M. A.; Grubbs, R. H.; Rosen, R. K.; Timmers, F. J. Organometallics 1996, 15, 1518-1520.

2. Clough, B. A.; Mellino, S.; Protchenko, A. V.; Slusarczyk, M.; Stevenson, L. C.; Blake, M. P.; Xie, B.; Clot, E.; Mountford, P. Inorg. Chem. 2017, 56, 10794-10814.

3. Ward, B. D.; Dubberley, S. R.; Maisse-Francois, A.; Gade, L. H.; Mountford, P. Dalton Trans. 2002, 4649.

4. Cochran, F. V.; Bonitatebus, P. J.; Schrock, R. R. Organometallics 2000, 19, 2414-2416.

5. Fauré, J.-L.; Gornitzka, H.; Réau, R.; Stalke, D.; Bertrand, G. Eur. J. Inorg. Chem. 1999, 2295-2299.

6. Galka, C. H.; Trösch, D. J. M.; Schubart, M.; Gade, L. H.; Radojevic, S.; Scowen, I. J.; McPartlin, M. Eur. J. Inorg. Chem. 2000, 2577.

7. (a) Nishikawa, T.; Ino, A.; Isobe, M. Tetrahedron 1994, 50, 1449-1468; (b) Zhang, Y.; Wen, J. J. Fluorine Chem. 1990, 47, 533-535.

8. Hadlington, T. J.; Abdalla, J. A. B.; Tirfoin, R.; Aldridge, S.; Jones, C. Chem. Commun. 2016, $52,1717-1720$.

9. (a) Otwinowski, Z.; Minor, W., Processing of X-ray Diffraction Data Collected in Oscillation Mode. Academic press: New York, 1997; (b) CrysAlisPro Agilent Technologies, Oxford, U.K.: 2011.

10. Altomare, A.; Cascarano, G.; Giacovazzo, G.; Guagliardi, A.; Burla, M. C.; Polidori, G.; Camalli, M. J. Appl. Crystallogr. 1994, 27, 435-435.

11. Palatinus, L.; Chapuis, G. J. Appl. Crystallogr. 2007, 40, 786-790.

12. Betteridge, P. W.; Cooper, J. R.; Cooper, R. I.; Prout, K.; Watkin, D. J. J. Appl. Crystallogr. 2003, 36, 1487-1487.

13. Frisch, M. J.; Trucks, G. W.; Schlegel, H. B.; Scuseria, G. E.; Robb, M. A.; Cheeseman, J. R.; Scalmani, G.; Barone, V.; Mennucci, B.; Petersson, G. A.; Nakatsuji, H.; Caricato, M.; Li, X.; Hratchian, H. P.; Izmaylov, A. F.; Bloino, J.; Zheng, G.; Sonnenberg, J. L.; Hada, M.; Ehara, M.; Toyota, K.; Fukuda, R.; Hasegawa, J.; Ishida, M.; Nakajima, T.; Honda, Y.; Kitao, O.; Nakai, H.; Vreven, T.; Montgomery Jr., J. A.; Peralta, J. E.; Ogliaro, F.; Bearpark, M. J.; Heyd, J.; Brothers, E. N.; Kudin, K. N.; Staroverov, V. N.; Kobayashi, R.; Normand, J.; Raghavachari, K.; Rendell, A. P.; Burant, J. C.; Iyengar, S. S.; Tomasi, J.; Cossi, M.; Rega, N.; Millam, N. J.; Klene, M.; Knox, J. E.; Cross, J. B.; Bakken, V.; Adamo, C.; Jaramillo, J.; Gomperts, R.; Stratmann, R. E.; Yazyev, O.; Austin, A. J.; Cammi, R.; Pomelli, C.; Ochterski, J. W.; Martin, R. L.; Morokuma, K.; Zakrzewski, V. G.; Voth, G. A.; Salvador, P.; Dannenberg, J. J.; Dapprich, S.; Daniels, A. D.; Farkas, Ö.; Foresman, J. B.; Ortiz, J. V.; 
Cioslowski, J.; Fox, D. J. Gaussian 09, Revision D.01, Gaussian, Inc.: Wallingford, CT, USA, 2009.

14. Weigend, F.; Ahlrichs, R. Phys. Chem. Chem. Phys. 2005, 7, 3297-3305.

15. Grimme, S.; Antony, J.; Ehrlich, S.; Krieg, H. J. Chem. Phys. 2010, 132, 154104-154119.

16. (a) Lee, A. M.; Handy, N. C.; Colwell, S. M. J. Chem. Phys. 1995, 103, 10095-10109; (b) Ditchfield, R. J. Chem. Phys. Lett. 1972, 56, 5688-5691.

17. Jensen, F. J. Chem. Theory Comput. 2015, 11, 132-138. 